\title{
Bromopyrrole Alkaloids of the Sponge Agelas oroides Collected Near the
}

\section{Israeli Mediterranean Coastline}

Dimitry Kovalerchik; ${ }^{\prime 1}$ Ravindra Pal Singh; ${ }^{2}$ Pnina Schlesinger $;{ }^{1}$ Aseel Mahajani; ${ }^{1}$ Micha Fridman $;{ }^{1}$ Micha Ilan; ${ }^{2}$ Shmuel Carmeli ${ }^{1 *}$

${ }^{1}$ Raymond and Beverly Sackler School of Chemistry and Faculty of Exact Sciences, Tel Aviv University, Ramat-Aviv 69978, Israel; ${ }^{2}$ School of

Zoology, George S. Wise Faculty of Life Sciences, Tel Aviv University, Ramat Aviv 69978, Israel

\section{Supporting Information}




\section{Table of content}

S1. Animal material

S2. Chart S1. Known brominated metabolites isolated in this study.

S3. ${ }^{1} \mathrm{H}$ NMR Spectrum of Agesamine C (1) in DMSO- $d_{6}$

S4. ${ }^{13} \mathrm{C}$ NMR Spectrum of Agesamine C (1) in DMSO- $d_{6}$

S5. HSQC Spectrum of Agesamine C (1) in DMSO- $d_{6}$

S6. HMBC Spectrum of Agesamine C (1) in DMSO- $d_{6}$

S7. COSY Spectrum of Agesamine C (1) in DMSO- $d_{6}$

S8. ROESY Spectrum of Agesamine C (1) in DMSO- $d_{6}$

S9. Table S1. NMR Data of Agesamine C (1) in DMSO- $d_{6}$

S10. HR ESI MS data of Agesamine C (1)

S11. ${ }^{1} \mathrm{H}$ NMR Spectrum of Dioroidamide A (2) in DMSO- $d_{6}$

S12. ${ }^{13} \mathrm{C}$ NMR Spectrum of Dioroidamide A (2) in DMSO- $d_{6}$

S13. HSQC Spectrum of Dioroidamide A (2) in DMSO- $d_{6}$

S14. HMBC Spectrum of Dioroidamide A (2) in DMSO- $d_{6}$

S15. COSY Spectrum of Dioroidamide A (2) in DMSO- $d_{6}$

S16. Table S2. NMR Data of Dioroidamide A (2) in DMSO- $d_{6}$

S17. Table S3. Comparison of the NMR data of Dioroidamide A (2) Mauritiamine, Oroidin, Cycloroidin and Najelamide J

S18. HR ESI MS data of Dioroidamide A (2)

S19. ${ }^{1} \mathrm{H}$ NMR Spectrum of Slagenin D (3) in DMSO- $d_{6}$

S20. ${ }^{13}$ C NMR Spectrum of Slagenin D (3) in DMSO- $d_{6}$

S21. HSQC Spectrum of Slagenin D (3) in DMSO- $d_{6}$

S22. HMBC Spectrum of Slagenin D (3) in DMSO- $d_{6}$

S23. COSY Spectrum of Slagenin D (3) in DMSO- $d_{6}$

S24. ROESY Spectrum of Slagenin D (3) in DMSO- $d_{6}$

S25. Table S4. NMR Data of Slagenin D (3) in DMSO- $d_{6}$

S26. Table S5. Comparison of the NMR Data $\left(500 \mathrm{MHz}, \mathrm{DMSO}-d_{6}\right)$ of Slagenin D (3), Slagenin A

S27. HR ESI MS data of Slagenin D (3) 
S28. ${ }^{1} \mathrm{H}$ NMR Spectrum of (-)-Monobromoagelaspongin (4) in DMSO- $d_{6}$

S29. ${ }^{13}$ C NMR Spectrum of (-)-Monobromoagelaspongin (4) in DMSO- $d_{6}$

S30. HSQC Spectrum of (-)-Monobromoagelaspongin (4) in DMSO- $d_{6}$

S31. HMBC Spectrum of (-)-Monobromoagelaspongin (4) in DMSO- $d_{6}$

S32. COSY Spectrum of (-)-Monobromoagelaspongin (4) in DMSO- $d_{6}$

S33. ${ }^{1} \mathrm{H}$ NMR Spectrum of (-)-Monobromoagelaspongin (4) in DMF- $d_{7}$ at $233{ }^{\circ} \mathrm{K}$

S34. Difference-NOE Spectra of (-)-Monobromoagelaspongin (4) in DMF- $d_{7}$ at $233{ }^{\circ} \mathrm{K}$

S35. Table S6. NMR Data of (-)-Monobromoagelaspongin (4) in DMSO- $d_{6}$ and in DMF- $d_{7}$ at $233{ }^{\circ} \mathrm{K}$

S36. HR ESI MS data of (-)-Monobromoagelaspongin (4)

S37. ${ }^{1} \mathrm{H}$ NMR Spectrum of (-)-11-Deoxymonobromoagelaspongin (5) in DMSO- $d_{6}$

S38. ${ }^{13} \mathrm{C}$ NMR Spectrum of (-)-11-Deoxymonobromoagelaspongin (5) in DMSO- $d_{6}$

S39. HSQC Spectrum of (-)-11-Deoxymonobromoagelaspongin (5) in DMSO- $d_{6}$

S40. HMBC Spectrum of (-)-11-Deoxymonobromoagelaspongin (5) in DMSO- $d_{6}$

S41. COSY Spectrum of (-)-11-Deoxymonobromoagelaspongin (5) in DMSO- $d_{6}$

S42. Table S7. NMR Data of (-)-11-Deoxymonobromoagelaspongin (5) in DMSO- $d_{6}$

S43. HR ESI MS data of (-)-11-Deoxymonobromoagelaspongin (5)

S44. ${ }^{1} \mathrm{H}$ NMR Spectrum of (-)-11-O-Methylmonobromoagelaspongin (6) in DMSO- $d_{6}$

S45. ${ }^{13} \mathrm{C}$ NMR Spectrum of (-)-11-O-Methylmonobromoagelaspongin (6) in DMSO- $d_{6}$

S46. HSQC Spectrum of (-)-11-O-Methylmonobromoagelaspongin (6) in DMSO- $d_{6}$

S47. HMBC Spectrum of (-)-11-O-Methylmonobromoagelaspongin (6) in DMSO- $d_{6}$

S48. COSY Spectrum of (-)-11-O-Methylmonobromoagelaspongin (6) in DMSO- $d_{6}$

S49. Table S8. NMR Data of (-)-11-O-Methylmonobromoagelaspongin (6) in DMSO- $d_{6}$

S50. HR ESI MS data of (-)-11-O-Methylmonobromoagelaspongin (6)

S51. ${ }^{1} \mathrm{H}$ NMR Spectrum of $E$-Dispacamide (7) in DMSO- $d_{6}$

S52. ${ }^{13} \mathrm{C}$ NMR Spectrum of $E$-Dispacamide (7) in DMSO- $d_{6}$

S53. HSQC Spectrum of $E$-Dispacamide (7) in DMSO- $d_{6}$

S54. HMBC Spectrum of $E$-Dispacamide (7) in DMSO- $d_{6}$ 
S55. COSY Spectrum of $E$-Dispacamide (7) in DMSO- $d_{6}$

S56. ROESY Spectrum of $E$-Dispacamide (7) in DMSO- $d_{6}$

S57. ${ }^{1} \mathrm{H}$ NMR Spectrum of $E$-Dispacamide (7) in DMF- $d_{7}$ at $233{ }^{\circ} \mathrm{K}$

S58. Difference-NOE Spectra of $E$-Dispacamide (7) in DMF- $d_{7}$ at $233^{\circ} \mathrm{K}$

S59. Table S9. NMR Data of $E$-Dispacamide (7) in DMSO- $d_{6}$ and in DMF- $d_{7}$ at $233{ }^{\circ} \mathrm{K}$

S60. Table S10. Comparison of the NMR Data (500 MHz, DMSO- $d_{6}$ ) for $E$-Dispacamide (7) and Dispacamide

S61. HR ESI MS data of $E$-Dispacamide (7)

S62. ${ }^{1} \mathrm{H}$ NMR Spectrum of Pyrrolosine (8) in DMSO- $d_{6}$

S63. ${ }^{13} \mathrm{C}$ NMR Spectrum of Pyrrolosine (8) in DMSO- $d_{6}$

S64. HSQC Spectrum of Pyrrolosine (8) in DMSO- $d_{6}$

S65. HMBC Spectrum of Pyrrolosine (8) in DMSO- $d_{6}$

S66. COSY Spectrum of Pyrrolosine (8) in DMSO- $d_{6}$

S67. Table S11. NMR Data of Pyrrolosine (8) in DMSO- $d_{6}$

S68. HR ESI MS data of Pyrrolosine (8)

S69. Physiochemical data of Agesamide A

S70. ${ }^{1} \mathrm{H}$ NMR Spectrum of Agesamide A in DMSO- $d_{6}$

S71. ${ }^{13} \mathrm{C}$ NMR Spectrum of Agesamide A in DMSO- $d_{6}$

S72. HSQC Spectrum of Agesamide A in DMSO- $d_{6}$

S73. HMBC Spectrum of Agesamide A in DMSO- $d_{6}$

S74. COSY Spectrum of Agesamide A in DMSO- $d_{6}$

S75. ROESY Spectrum of Agesamide A in DMSO- $d_{6}$

S76. Table S12. NMR Data of Agesamide A in DMSO- $d_{6}$

S77. HR ESI MS data of Agesamide A

S78. Physiochemical data of Agesamide B

S79. ${ }^{1} \mathrm{H}$ NMR Spectrum of Agesamide B in DMSO- $d_{6}$

S80. ${ }^{13} \mathrm{C}$ NMR Spectrum of Agesamide B in DMSO- $d_{6}$

S81. HSQC Spectrum of Agesamide B in DMSO- $d_{6}$ 
S82. HMBC Spectrum of Agesamide B in DMSO- $d_{6}$

S83. COSY Spectrum of Agesamide B in DMSO- $d_{6}$

S84. ROESY Spectrum of Agesamide B in DMSO- $d_{6}$

S85. Table S13. NMR Data of Agesamide B in DMSO- $d_{6}$

S86. HR ESI MS data of Agesamide B

S87. Physiochemical data of Agesamine A

S88. ${ }^{1} \mathrm{H}$ NMR Spectrum of Agesamine A in DMSO-d6

S89. ${ }^{13} \mathrm{C}$ NMR Spectrum of Agesamine A in DMSO-d6

S90. HSQC Spectrum of Agesamine A in DMSO-d6

S91. HMBC Spectrum of Agesamine A in DMSO- $d_{6}$

S92. COSY Spectrum of Agesamine A in DMSO- $d_{6}$

S93. Difference-NOE Spectra of Agesamine A in DMSO- $d_{6}$

S94. Table S14. NMR Data of Agesamine A in DMSO- $d_{6}$

S95. HR ESI MS data of Agesamine A

S96. Physiochemical data of Agesamine B

S97. ${ }^{1} \mathrm{H}$ NMR Spectrum of Agesamine B in DMSO- $d_{6}$

S98. ${ }^{13}$ C NMR Spectrum of Agesamine B in DMSO- $d_{6}$

S99. HSQC Spectrum of Agesamine B in DMSO- $d_{6}$

S100. HMBC Spectrum of Agesamine B in DMSO- $d_{6}$

S101. COSY Spectrum of Agesamine B in DMSO- $d_{6}$

S102. Difference-NOE Spectra of Agesamine B in DMSO- $d_{6}$

S103. Table S15. NMR Data of Agesamine B in DMSO- $d_{6}$

S104. HR ESI MS data of Agesamine B 
S1. Animal material

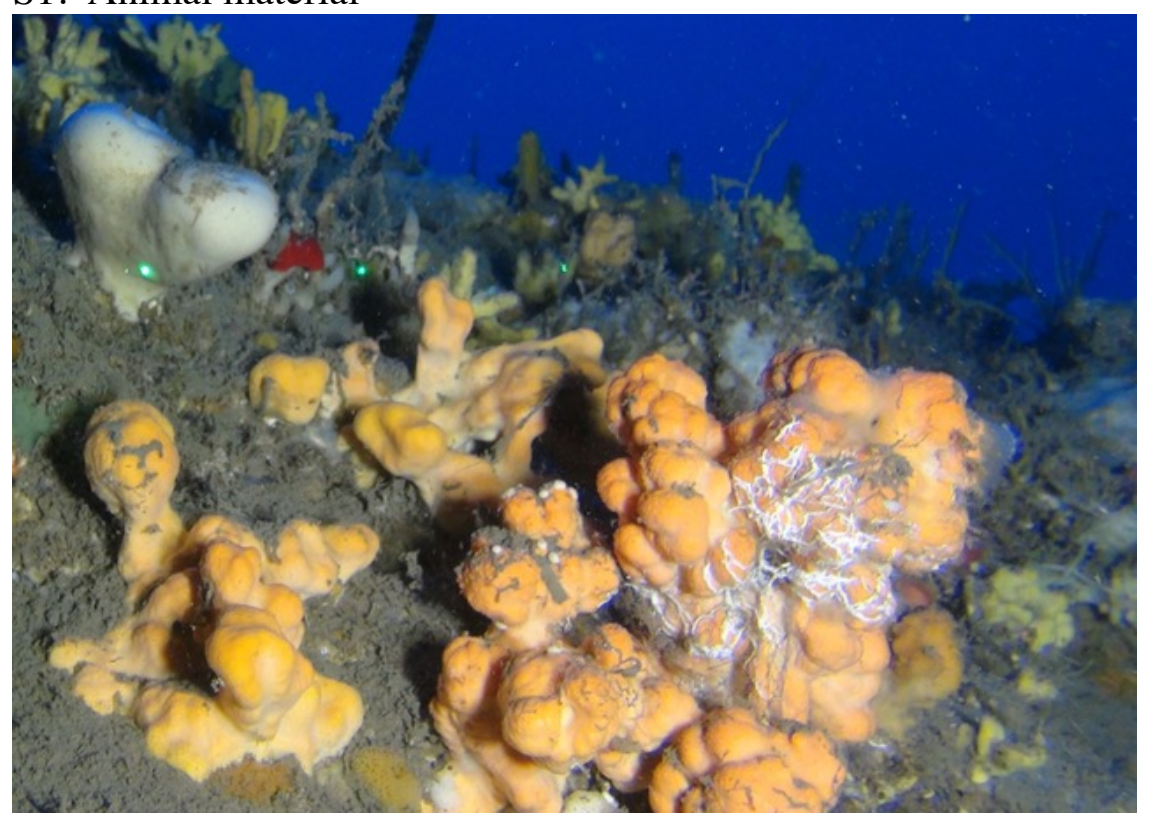

The photograph of the sponge Agelas oroides was taken by Tal Idan, at a depth of $100 \mathrm{~m}$ using ROV. 
S2. Chart S1. Known brominated metabolites isolated in this study.

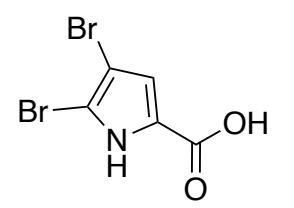

4,5-dibromopyrrole2-carboxylic acid

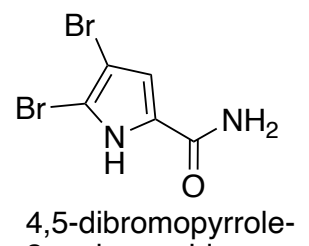

2-carboxamide

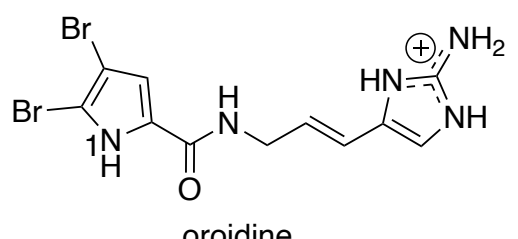

oroidine

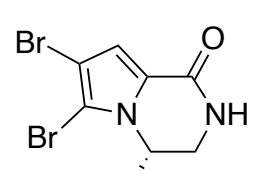

$\mathrm{HO}$

longamide A<smiles>CNCCCOc1c(Br)cc(CCN(C)C)cc1Br</smiles>

purealidin $\mathrm{F}$<smiles>N[C@@H]1NC(=O)/C(=C/CCNC(=O)c2cc(Br)c(Br)[nH]2)N1</smiles>

dispacamide<smiles>N=C1[N-]C(=CC(O)CNC(=O)c2cc(Br)c(Br)[nH]2)C(=O)N1</smiles>

dispacamide $\mathrm{C}$

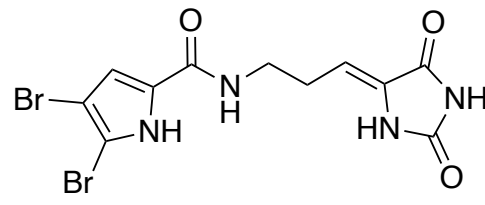

mukanadin D

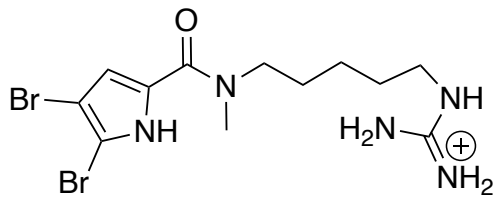

mukanadin $\mathrm{E}$<smiles></smiles>

taurodispacamide $\mathrm{A}$

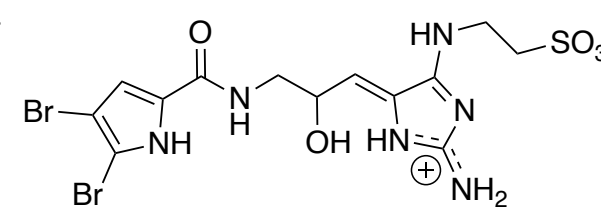

tauroacdin A

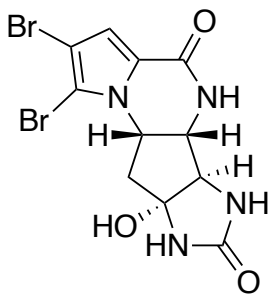

agelastatin $\mathrm{F}$

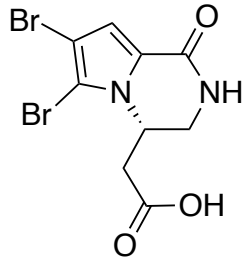

longamide B

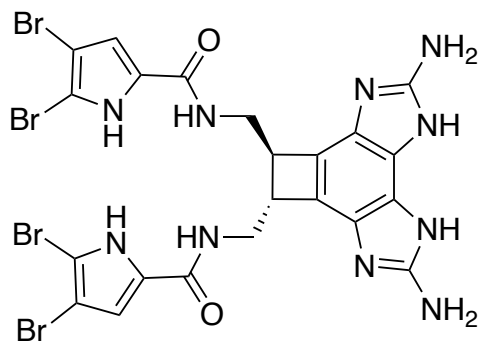

benzosceptin $C$

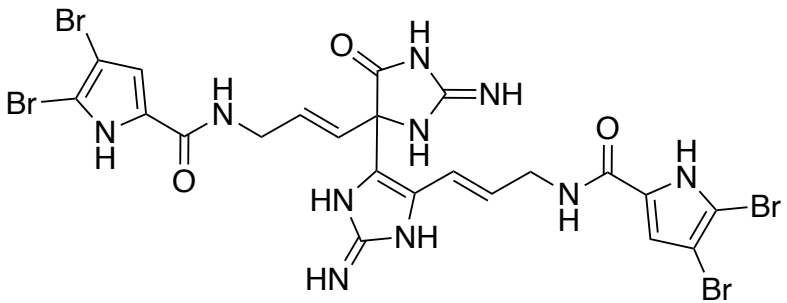

mauritiamine

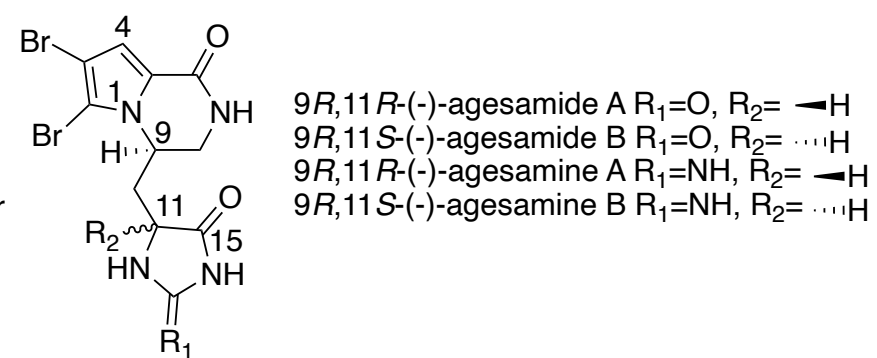


S3. ${ }^{1} \mathrm{H}$ NMR Spectrum of Agesamine C (1) in DMSO- $d_{6}$

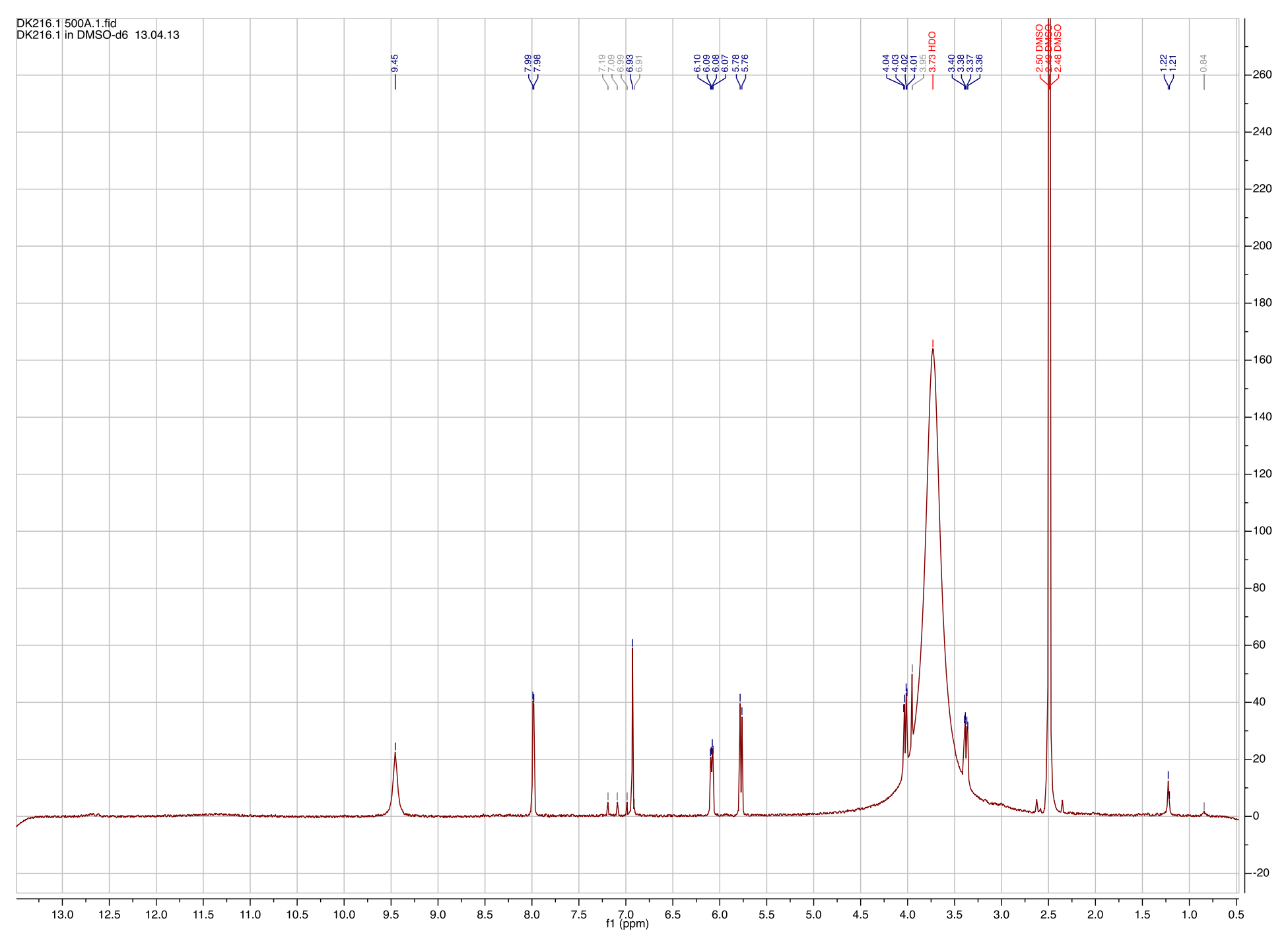


S4. ${ }^{13} \mathrm{C}$ NMR Spectrum of Agesamine C (1) in DMSO- $d_{6}$

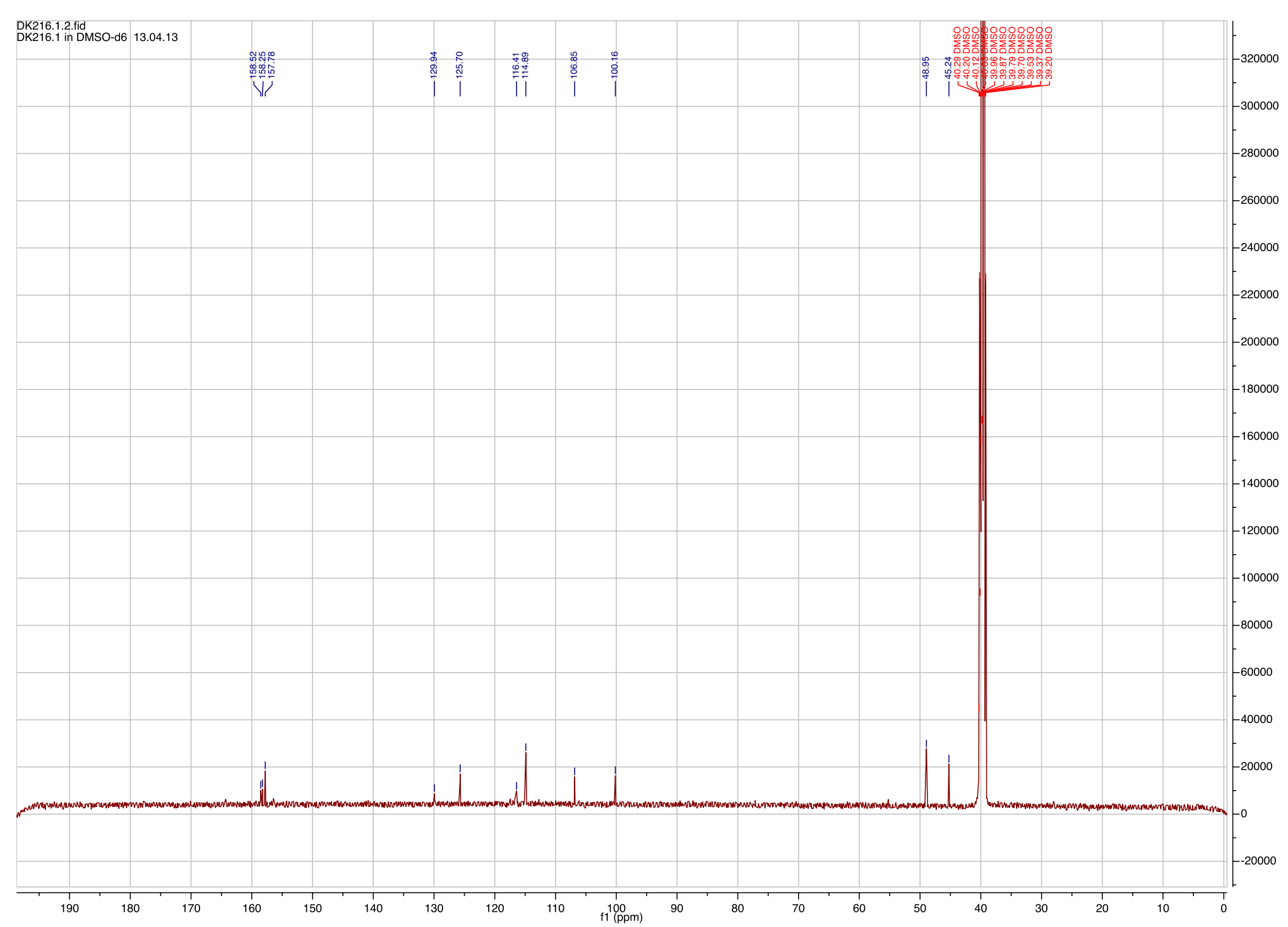


S5. HSQC Spectrum of Agesamine C (1) in DMSO- $d_{6}$

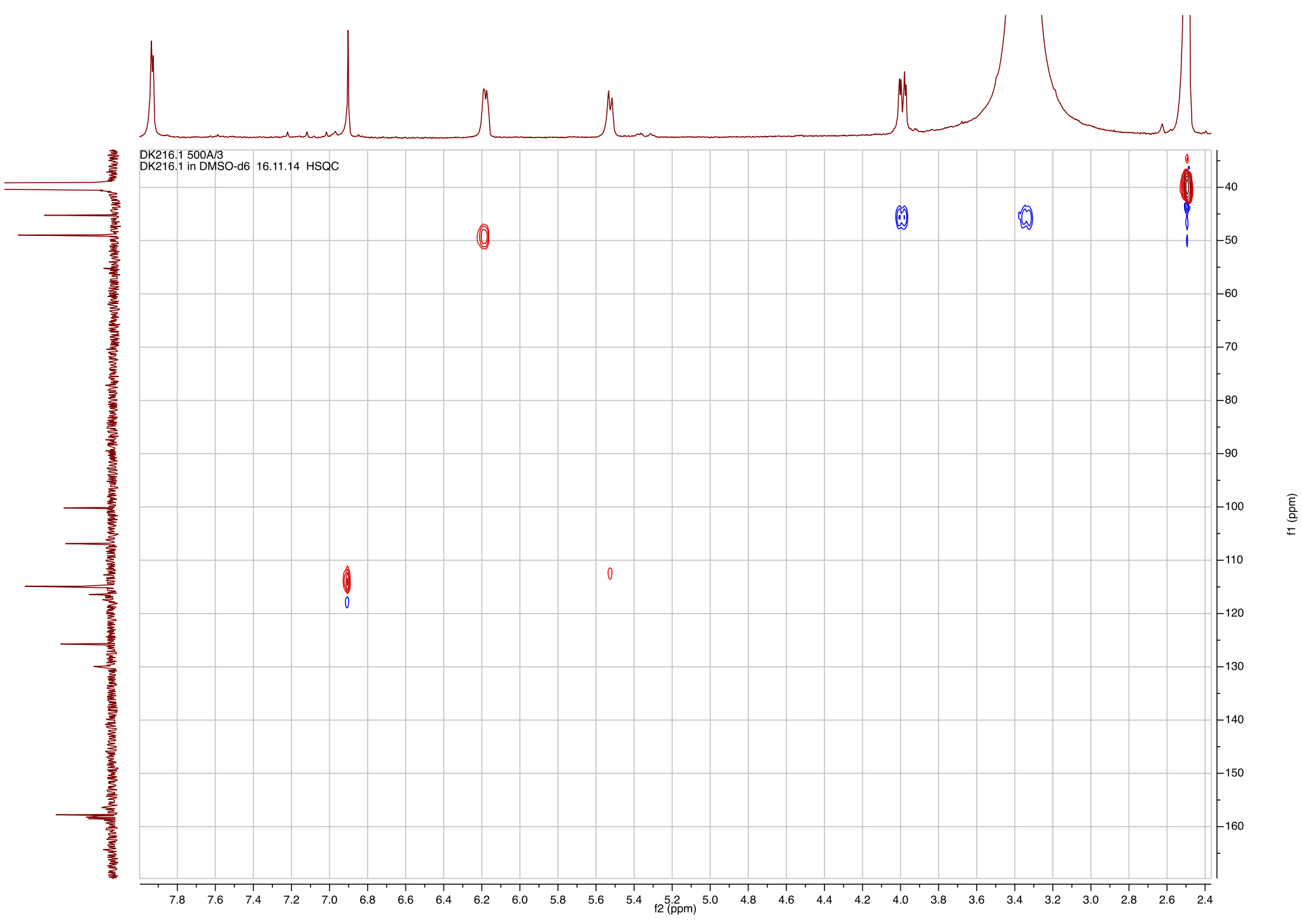


S6. HMBC Spectrum of Agesamine C (1) in DMSO- $d_{6}$

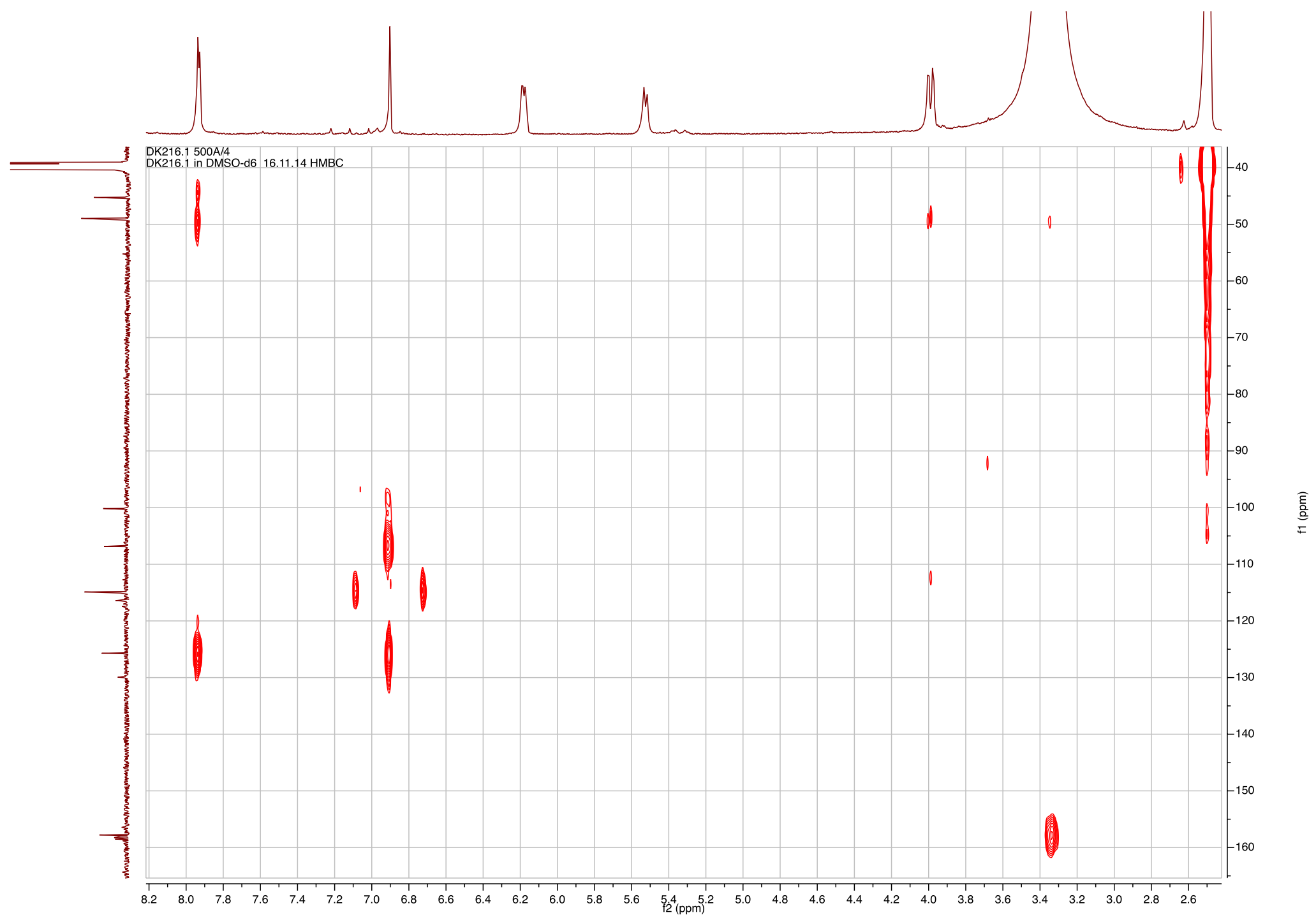


S7. COSY Spectrum of Agesamine C (1) in DMSO- $d_{6}$

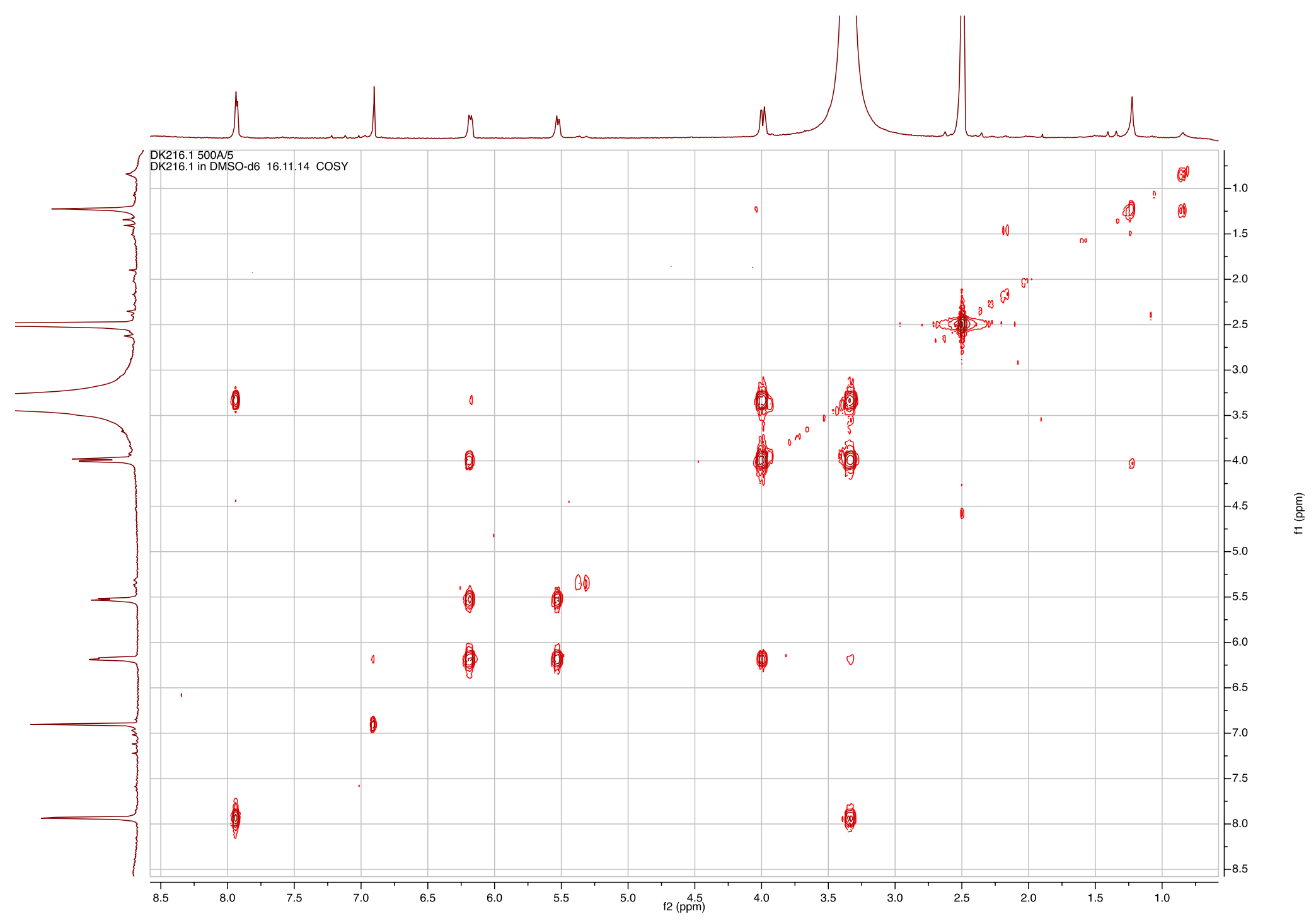


S8. ROESY Spectrum of Agesamine C (1) in DMSO- $d_{6}$

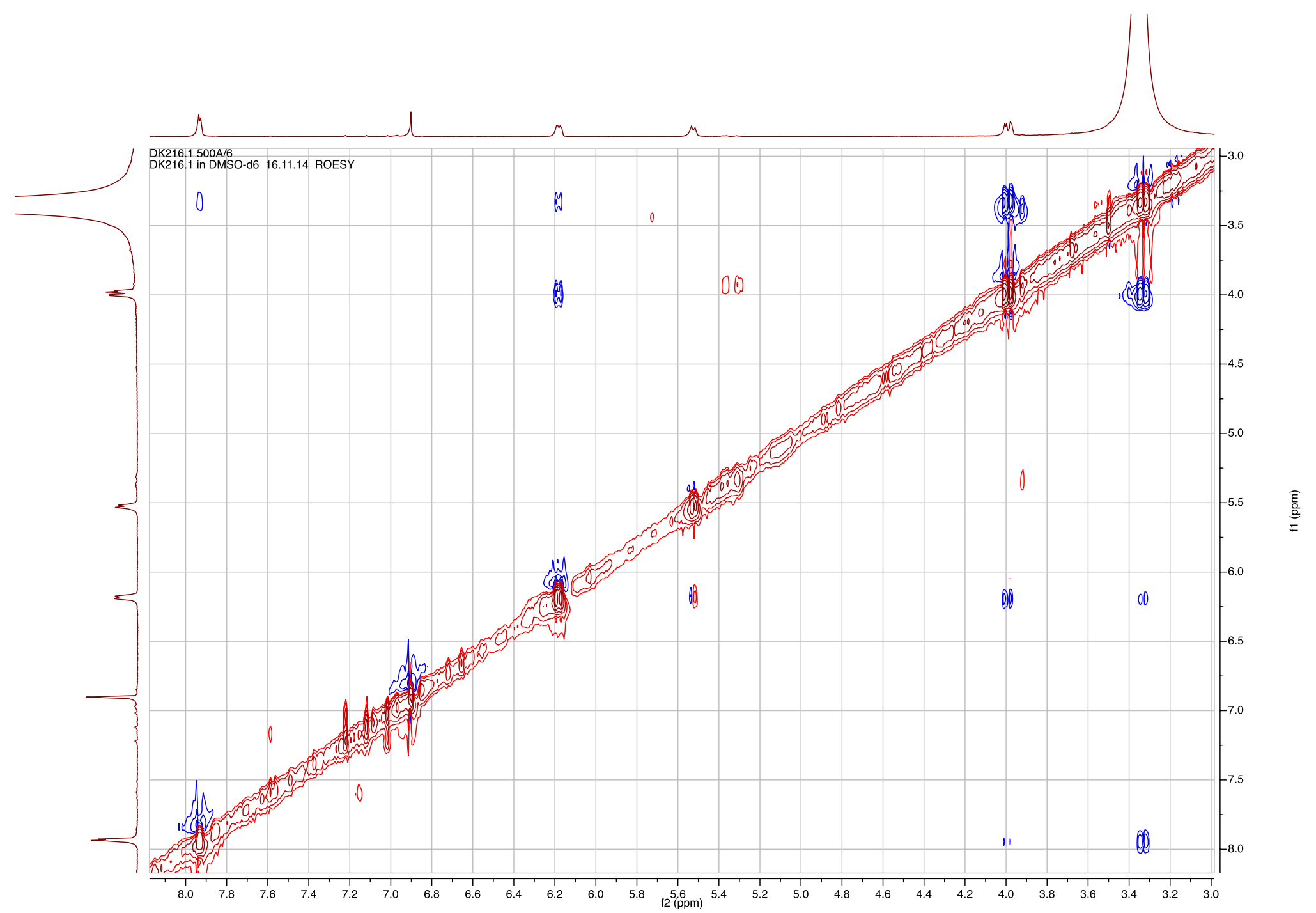


S9. Table S1. NMR data of Agesamine C (1) in DMSO- $d_{6}{ }^{\mathrm{a}}$

\begin{tabular}{lllll}
\hline Position & $\delta_{\mathrm{C}} \mathrm{b}$ & $\delta_{\mathrm{H}}$, mult., $J(\mathrm{~Hz})$ & HMBC correlations & NOE correlations \\
\hline 2 & $106.9, \mathrm{C}$ & & 4 & \\
3 & $100.2, \mathrm{C}$ & & & \\
4 & $114.9, \mathrm{CH}$ & $6.92, \mathrm{~s}$ & 4,7 & \\
5 & $125.7, \mathrm{C}$ & & $8 \mathrm{~b}$ & $8 \mathrm{a}, 8 \mathrm{~b}$ \\
6 & $157.8, \mathrm{C}$ & & & $7,9,10,12$ \\
7 & & $7.98, \mathrm{~d}(5.0)$ & $7,9,12$ \\
$8 \mathrm{a}$ & $45.2, \mathrm{CH} 2$ & $4.01, \mathrm{dd}(13.1,4.0)$ & 7 & $8 \mathrm{a}, 8 \mathrm{~b}$ \\
$\mathrm{~b}$ & & $3.37, \mathrm{dd}(13.1,5.0)$ & & $8 \mathrm{a}$ \\
9 & $49.0, \mathrm{CH}$ & $6.15, \mathrm{dd}(9.2,4.0)$ & $7,8 \mathrm{a}, 8 \mathrm{~b}$ & $8 \mathrm{a}, 8 \mathrm{~b}$ \\
10 & $116.4, \mathrm{CH}$ & $5.76, \mathrm{~d}(9.2)$ & & \\
11 & $129.9, \mathrm{C}$ & & & \\
12 & & $9.45, \mathrm{brs}$ & \\
13 & $156.4, \mathrm{C}$ & & & \\
$13-\mathrm{NH}$ & & 9.45, brs & & \\
14 & & 9.45, brs & & \\
15 & $164.4, \mathrm{C}$ & & & \\
\hline $\mathrm{a} 500.13 \mathrm{MHz}$ for ${ }^{1} \mathrm{H}$ and $125.76 \mathrm{MHz}$ for ${ }^{13} \mathrm{C} .{ }^{\mathrm{b}}$ Multiplicity and assignment from HSQC experiment.
\end{tabular}


S10. HR ESI MS data of Agesamine C (1)

\section{Elemental Composition Report}

Page 1

Single Mass Analysis

Tolerance $=5.0 \mathrm{mDa} / \mathrm{DBE} \cdot \mathrm{min}=-1.5, \max =50.0$

of isotope peaks used for $\mathrm{i}-\mathrm{FIT}=3$

Monoisotopic Mass, Even Electron lons

266 formula(e) evaluated with 9 results within limits (up to 4 closest results for each mass)

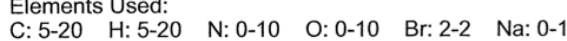

DK216.1
carmeli919 29 (1.298) Cm (29:34)

Dimitry Kovalerchik

1: TOF MS ES+

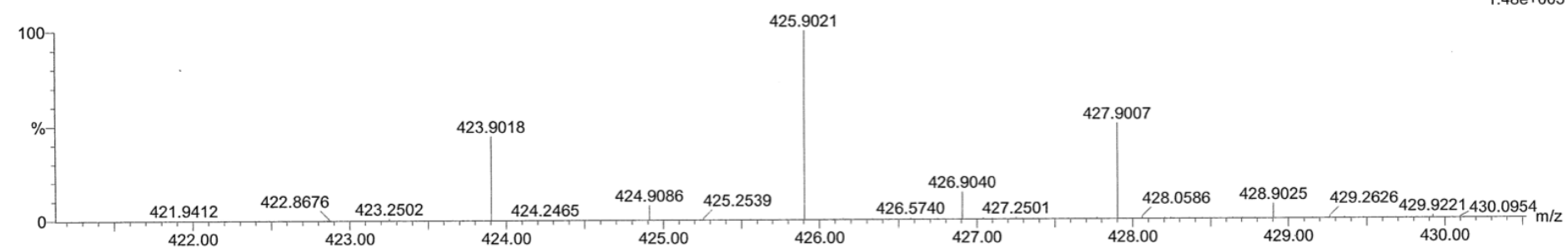

Minimum:

Maximum: $5.0 \quad 10.0 \quad 500$

Mass Calc. Mass mDa PPM DBE

$423.9018 \quad 423.9021$

423.9021
423.900
423

$\begin{array}{lll}-0.3 & -0.7 & 8.5 \\ -1.3 & 2.6 & -3.1\end{array}$

423.9004

i-FIT i-FIT (Norm) Formula

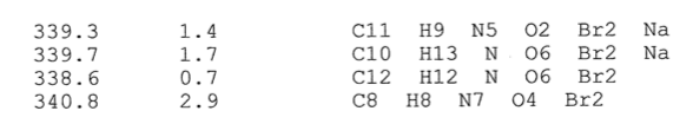


S11. ${ }^{1} \mathrm{H}$ NMR Spectrum of Dioroidamide A (2) in DMSO- $d_{6}$

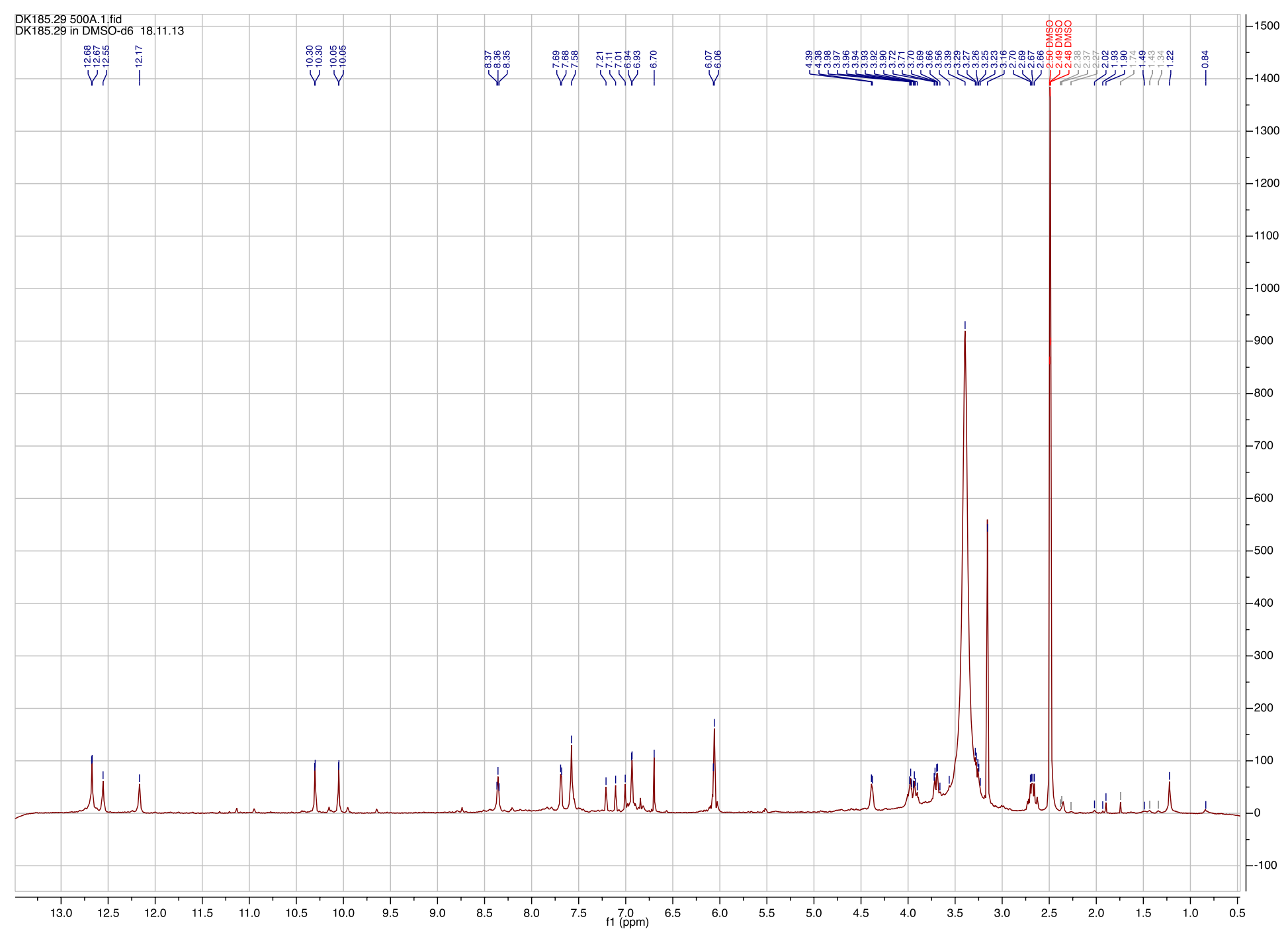


S12. ${ }^{13} \mathrm{C}$ NMR Spectrum of Dioroidamide A (2) in DMSO- $d_{6}$
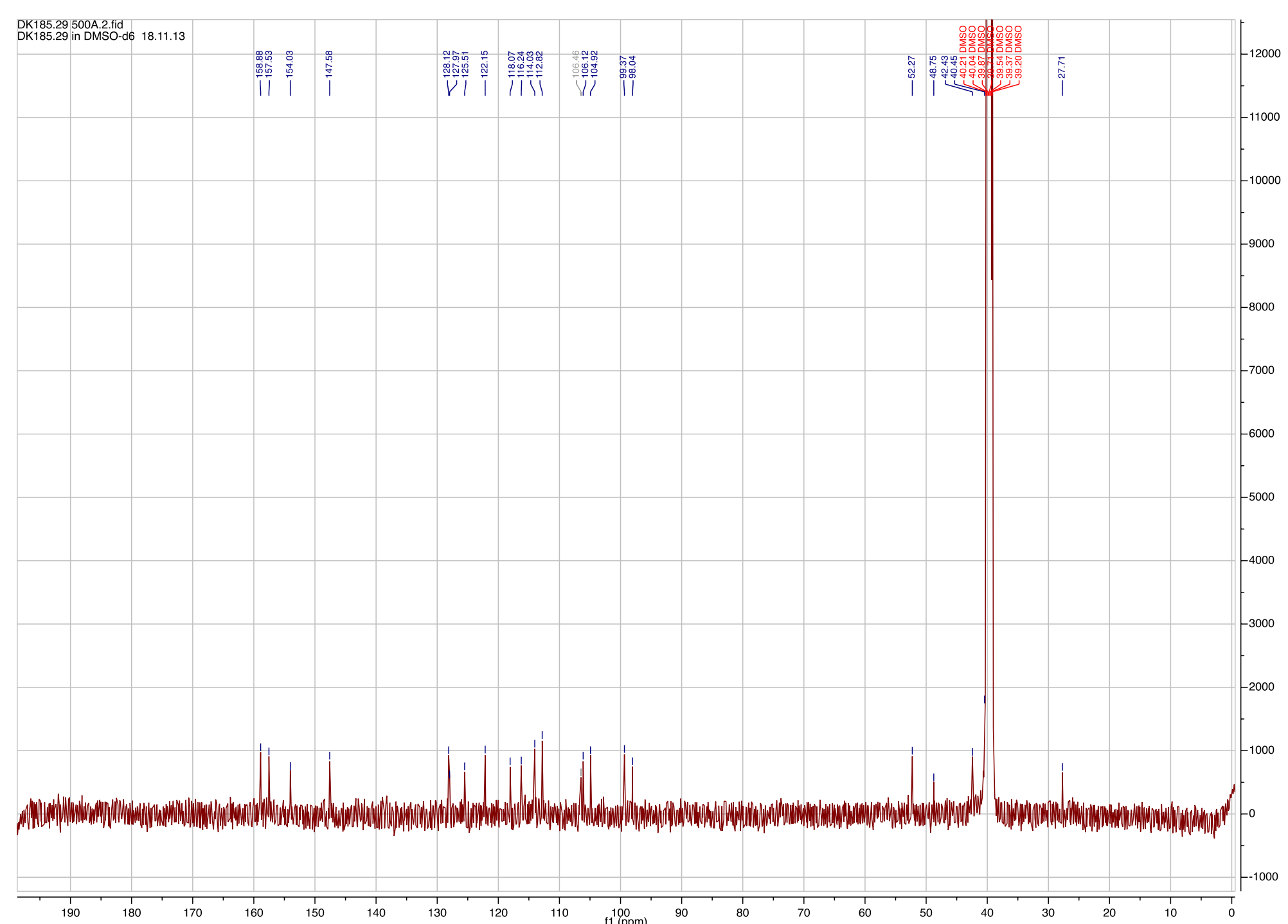
S13. HSQC Spectrum of Dioroidamide A (2) in DMSO- $d_{6}$

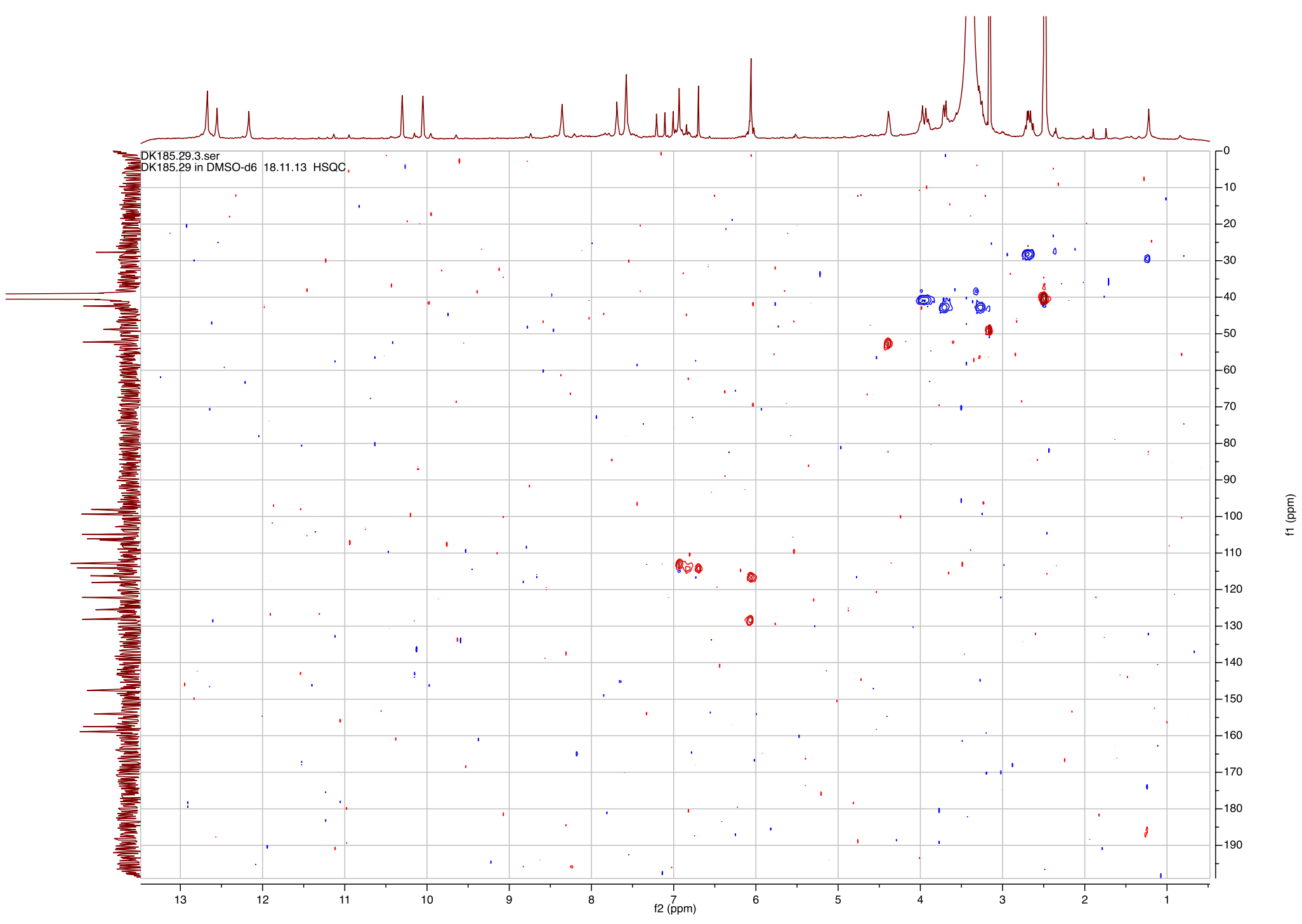


S14. HMBC Spectrum of Dioroidamide A (2) in DMSO- $d_{6}$

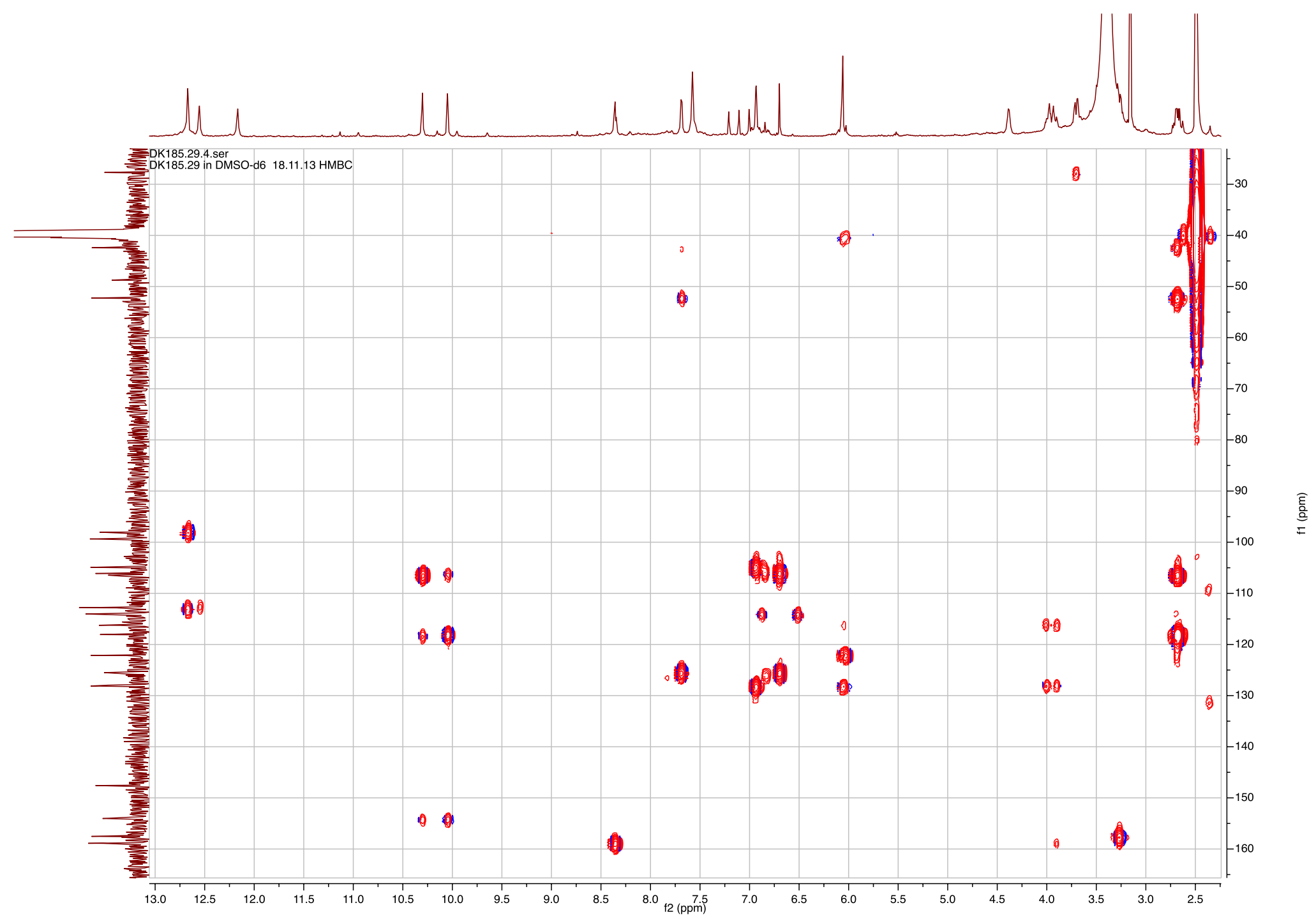


S15. COSY Spectrum of Dioroidamide A (2) in DMSO- $d_{6}$

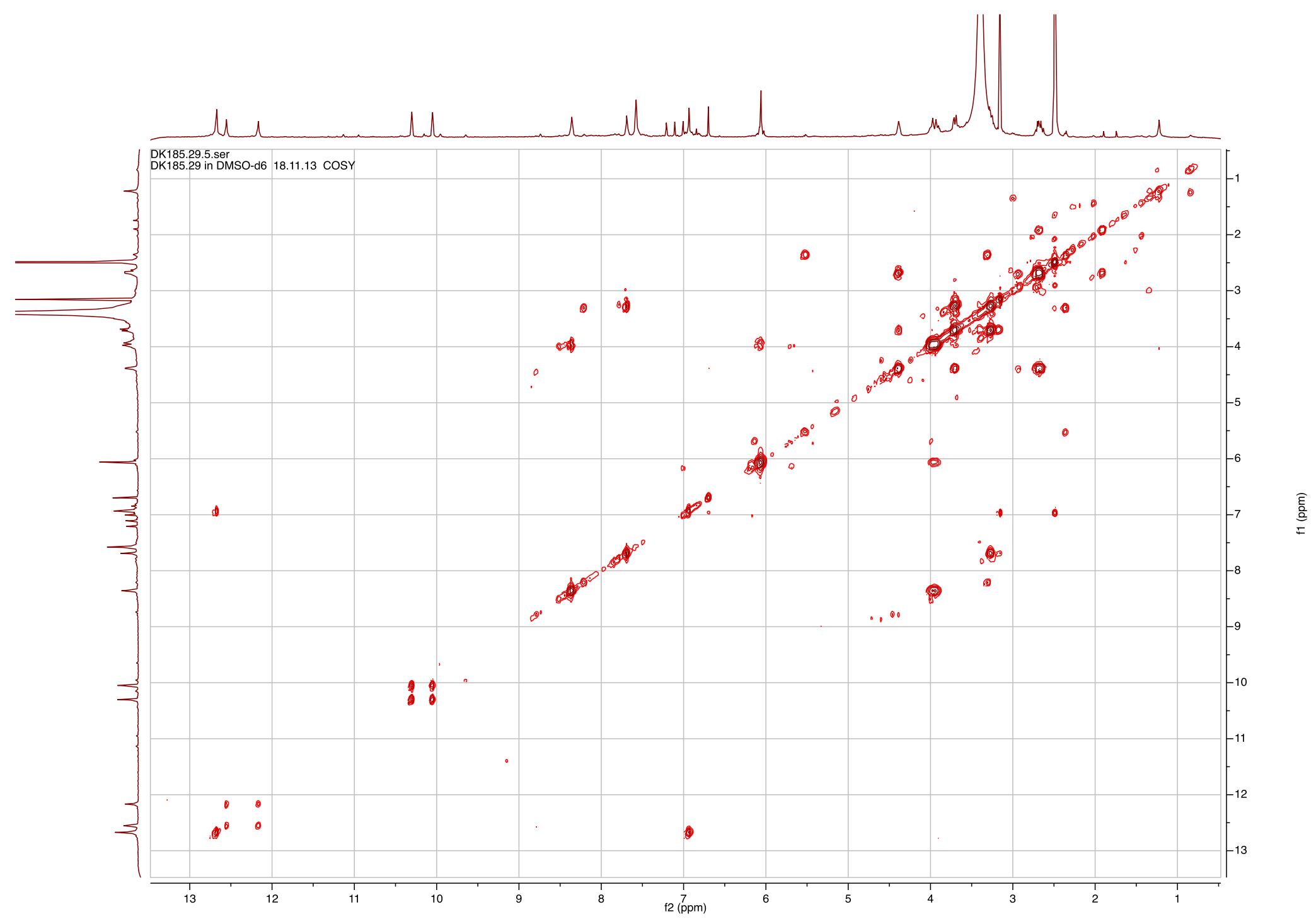


S16. Table S2. NMR data of Dioroidamide A (2) in DMSO- $d_{6}{ }^{\mathrm{a}}$

\begin{tabular}{|c|c|c|c|c|}
\hline Position & $\delta_{\mathrm{C}}^{\mathrm{b}}$ & $\delta_{\mathrm{H}}$, mult., $J(\mathrm{~Hz})$ & HMBC correlations & NOE correlations \\
\hline 1 & & $12.67, \mathrm{~s}$ & & \\
\hline 2 & 104.9, C & & 4 & \\
\hline 3 & $98.4, \mathrm{C}$ & & 1 & \\
\hline 4 & $112.8, \mathrm{CH}$ & $6.94, \mathrm{~d}(2.0)$ & 1 & \\
\hline 5 & 128.1, C & & $4,7,8$ & \\
\hline 6 & 158.9, C & & 7 & \\
\hline 7 & & $8.36, \mathrm{t}(5.5)$ & & \\
\hline 8 & $40.5, \mathrm{CH}_{2}$ & $3.95, \mathrm{~m}$ & 10 & 10 \\
\hline 9 & $128.0, \mathrm{CH}$ & $6.07, \mathrm{~m}$ & 8 & 12 \\
\hline 10 & $116.3, \mathrm{CH}$ & $6.06, \mathrm{~m}$ & 8 & 8 \\
\hline 11 & $122.2, \mathrm{C}$ & & 10,13 & \\
\hline 12 & & $12.55, \mathrm{~s}$ & & 9 \\
\hline 13 & 147.6, C & & 12 & \\
\hline $13-\mathrm{NH}_{2}$ & & $7.58, \mathrm{~s}$ & & \\
\hline 14 & & $12.17, \mathrm{~s}$ & & \\
\hline 15 & 112.6, C & & 12 & \\
\hline 2 & 106.5, C & & $4^{\prime}, 9^{\prime}$ & \\
\hline $3^{\prime}$ & $99.4, \mathrm{C}$ & & & \\
\hline 4 & $114.0, \mathrm{CH}$ & $6.70, \mathrm{~s}$ & & \\
\hline 5 & $125.5, \mathrm{C}$ & & 9' & \\
\hline 6 ' & 157.5, C & & 8 & \\
\hline 7 & & $7.68 \mathrm{~d}(4.5)$ & & \\
\hline $\begin{array}{r}8 ' \mathrm{a} \\
\mathrm{b}\end{array}$ & $42.4, \mathrm{CH}_{2}$ & $\begin{array}{l}3.25, \mathrm{~m} \\
3.69 \mathrm{dd}(13.0,4.5)\end{array}$ & $7 ', 10^{\prime}$ & \\
\hline 9' & $52.3, \mathrm{CH}$ & $4.38 \mathrm{dd}(11.1,6.7)$ & $10^{\prime}$ & $12^{\prime}$ \\
\hline
\end{tabular}




\begin{tabular}{lllll}
$10^{\prime}$ & $27.7, \mathrm{CH}_{2}$ & $2.67, \mathrm{~m}$ & $8^{\prime}$ & $12^{\prime}$ \\
$11^{\prime}$ & $106.1, \mathrm{C}$ & & $10^{\prime}, 14^{\prime}, 12^{\prime}$ & \\
$12^{\prime}$ & & $10.30, \mathrm{~s}$ & & \\
$13^{\prime}$ & $154.0, \mathrm{C}$ & & $14^{\prime}, 12^{\prime}$ & \\
$14^{\prime}$ & & $10.05, \mathrm{~s}$ & & \\
$15^{\prime}$ & $118.1, \mathrm{C}$ & & $10^{\prime}, 14^{\prime}, 12^{\prime}$ & $9^{\prime}, 10^{\prime}$ \\
\hline
\end{tabular}

${ }^{\mathrm{a}} 500.13 \mathrm{MHz}$ for ${ }^{1} \mathrm{H}$ and $125.76 \mathrm{MHz}$ for ${ }^{13} \mathrm{C}$. ${ }^{\mathrm{b}}$ Multiplicity and assignment from HSQC experiment.

S17. Table S3. Comparison of the NMR data of Dioroidamide A (2) Mauritiamine, Oroidin, Cycloroidin and Najelamide Ja

\begin{tabular}{|c|c|c|c|c|c|c|c|c|}
\hline \multirow[t]{2}{*}{ Position } & \multicolumn{2}{|c|}{ Dioroidamide A (6) (DMSO- $d_{6}$ ) } & \multicolumn{2}{|c|}{ Mauritiamine $\left(\mathrm{CD}_{3} \mathrm{OD}\right)$} & \multicolumn{2}{|c|}{$\begin{array}{l}\text { Oroidin }\left(\mathrm{DMSO}-d_{6}\right) \\
\text { Cyclooroidin }\left(\mathrm{CD}_{3} \mathrm{OD}\right)^{24 \mathrm{a}}\end{array}$} & \multicolumn{2}{|c|}{ Najelamide $\mathrm{J}\left(\mathrm{DMSO}-d_{6}\right)^{25}$} \\
\hline & $\delta_{C}{ }^{b}$ & $\delta_{\mathrm{H}}$, mult., $J(\mathrm{~Hz})$ & $\delta_{C}^{b}$ & $\delta_{\mathrm{H}}$, mult., J (Hz) & $\delta_{C}^{b}$ & $\delta_{\mathrm{H}}$, mult., $J(\mathrm{~Hz})$ & $\delta_{C}^{b}$ & $\delta_{\mathrm{H}}$, mult., $J(\mathrm{~Hz})$ \\
\hline 1 & & $12.67, \mathrm{~s}$ & & & & $12.70, \mathrm{~s}$ & & $12.65, \mathrm{brs}$ \\
\hline 2 & 104.9, C & & 106.7, C & & $104.9, \mathrm{C}$ & & 104.5, C & \\
\hline 3 & $98.4, \mathrm{C}$ & & $100.2, \mathrm{C}$ & & $98.1, \mathrm{C}$ & & $97.8, \mathrm{C}$ & \\
\hline 4 & $112.8, \mathrm{CH}$ & $6.94, \mathrm{~d}(2.0)$ & $114.8, \mathrm{CH}$ & $6.89, \mathrm{~s}$ & $112.9, \mathrm{CH}$ & 6.96 , brs & $112.5, \mathrm{CH}$ & $6.89, \mathrm{~s}$ \\
\hline 5 & 128.1, C & & 128.7, C & & $128.2, \mathrm{C}$ & & 128.1, C & \\
\hline 6 & 158.9, C & & 162.0, C & & $158.9, \mathrm{C}$ & & 158.9, C & \\
\hline 7 & & $8.36, \mathrm{t}(5.5)$ & & & & $8.44, \mathrm{t}(5.7)$ & & $8.20, \mathrm{t}(5.6)$ \\
\hline $\begin{array}{r}8 \mathrm{a} \\
\mathrm{b}\end{array}$ & $40.5, \mathrm{CH}_{2}$ & $3.95, \mathrm{~m}$ & $42.1 \mathrm{CH}_{2}$ & $\begin{array}{l}4.10 \mathrm{dd}(16.0,5.5) \\
3.93 \mathrm{dd}(16.0,5.5)\end{array}$ & $39.9, \mathrm{CH}_{2}$ & $3.95, \operatorname{brdd}(5.7,5.3)$ & $38.9, \mathrm{CH}_{2}$ & $3.21, \mathrm{~m}$ \\
\hline 9 & $128.0, \mathrm{CH}$ & $6.07, \mathrm{~m}$ & $131.3, \mathrm{CH}$ & $6.11 \mathrm{dt}(16.5,5.5)$ & $126.7, \mathrm{CH}$ & $6.08, \mathrm{dt}(16.2,5.3)$ & $28.8, \mathrm{CH}_{2}$ & $1.73, \mathrm{~m}$ \\
\hline 10 & $116.3, \mathrm{CH}$ & $6.06, \mathrm{~m}$ & $116.9, \mathrm{CH}$ & $6.42 \mathrm{~d}(16.5)$ & $116.5, \mathrm{CH}$ & $6.21, d(16.2)$ & $21.9, \mathrm{CH}_{2}$ & $2.58, \mathrm{~m}$ \\
\hline 11 & $122.2, \mathrm{C}$ & & $125.6 \mathrm{C}$ & & $125.0, \mathrm{C}$ & & 126.7, C & \\
\hline
\end{tabular}




\begin{tabular}{|c|c|c|c|c|c|c|c|c|}
\hline 12 & & $12.55, \mathrm{~s}$ & & & & $12.73, \mathrm{~s}$ & & $12.62, \mathrm{brs}$ \\
\hline 13 & 147.6, C & & 149.4, C & & 148.0, C & & 146.9, C & \\
\hline $13-\mathrm{NH}_{2}$ & & $7.58, \mathrm{~s}$ & & & & $7.65, \mathrm{~s}$ & & 7.43, brs \\
\hline 14 & & $12.17, \mathrm{~s}$ & & & & $12.02, \mathrm{~s}$ & & 11.50, brs \\
\hline 15 & 112.6, C & & $119.5, \mathrm{C}$ & & $112.4, \mathrm{CH}$ & 6.90, brs & $113.9, \mathrm{C}$ & \\
\hline $2^{\prime}$ & 106.5, C & & 106.6, C & & 111.4, C & & 105.3, C & \\
\hline 3 , & $99.4, \mathrm{C}$ & & 100.3, C & & $99.3, \mathrm{C}$ & & $98.0, \mathrm{C}$ & \\
\hline 4' & $114.0, \mathrm{CH}$ & $6.70, \mathrm{~s}$ & $114.8, \mathrm{CH}$ & $6.86, \mathrm{~s}$ & $115.1, \mathrm{CH}$ & $6.97 \mathrm{~s}$ & $113.6, \mathrm{CH}$ & $7.02, \mathrm{~s}$ \\
\hline 5 & 125.5, C & & 128.6, C & & $128.5, \mathrm{C}$ & & 127.4, C & \\
\hline 6 & 157.5, C & & $161.8, \mathrm{C}$ & & 163.5, C & & 158.6, C & \\
\hline $7^{\prime}$ & & $7.68 \mathrm{~d}(4.5)$ & & & & & & $8.68, \mathrm{~d}(7.0)$ \\
\hline 8'a & $42.4, \mathrm{CH}_{2}$ & $3.25, \mathrm{~m}$ & $42.1, \mathrm{CH}_{2}$ & $4.03 \mathrm{dd}(16.5,5.0)$ & $45.6, \mathrm{CH}_{2}$ & $3.78, \mathrm{dd}(13.6,3.5)$ & $60.3, \mathrm{CH}$ & $4.82, \mathrm{~m}$ \\
\hline $\mathrm{b}$ & & $3.69 \mathrm{dd}(13.0,4.5)$ & & $3.93 \mathrm{dd}(16.5,5.0)$ & & $3.55, \mathrm{dd}(13.6,1.2)$ & & \\
\hline 9' & $52.3, \mathrm{CH}$ & $4.38 \mathrm{dd}(11.1,6.7)$ & $133.2, \mathrm{CH}$ & $6.03 \mathrm{dt}(15.5,5.0)$ & $57.9, \mathrm{CH}$ & & $130.9, \mathrm{CH}$ & $6.08, \mathrm{~d}(2.2)$ \\
\hline $10^{\prime}$ & 27.7, $\mathrm{CH}_{2}$ & $2.67, \mathrm{~m}$ & 127.0, $\mathrm{CH}$ & $5.91 \mathrm{~d}(15.5)$ & $33.2, \mathrm{CH}_{2}$ & 2.81 brd (6.3) & 137.4, C & \\
\hline $11^{\prime}$ & 106.1, C & & $66.8, \mathrm{C}$ & & $132.9, \mathrm{C}$ & & 103.5, C & \\
\hline $12^{\prime}$ & & $10.30, \mathrm{~s}$ & & & & & & $9.71 \mathrm{brs}$ \\
\hline $13{ }^{\prime}$ & 154.0, C & & 163.0, C & & 153.9, C & & 157.2, C & \\
\hline $14^{\prime}$ & & $10.05, \mathrm{~s}$ & & & & & & 9.18, brs \\
\hline 15 , & 118.1, C & & 178.6, C & & $114.8, \mathrm{CH}$ & $6.32, \mathrm{~s}$ & $66.8, \mathrm{CH}$ & $4.31, \mathrm{brs}$ \\
\hline
\end{tabular}


S18. HR ESI MS data of Dioroidamide A (2)

Elemental Composition Report

Page 1

Single Mass Analysis

Tolerance $=4.0$ PPM / DBE: $\min =-1.5, \max =50.0$

Number of isotope peaks used for $\mathrm{i}-\mathrm{FIT}=3$

Monoisotopic Mass, Even Electron lons
883 formula(e) evaluated with 6 results within limits (up to 50 closest results for each mass)

Elements Used:
C: $17-27 \quad$ H: $10-30 \quad N: 4-14 \quad$ O: $0-10 \quad$ Br: 0-6

DK185.29

Drmeli908d $183(3.454)$

Dimitry Kovalerchik

100

TOF MS ES+
$4.90 \mathrm{e}+003$

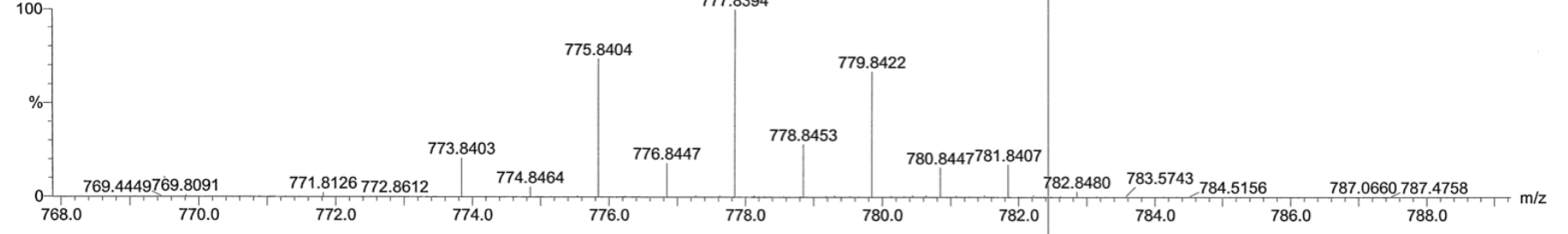

Calc. Mass

$\mathrm{MDa} \quad \mathrm{PPM} \quad \mathrm{DB}$

773.8403

$\begin{array}{llll}773.8423 & -2.0 & -2.6 & 15 . \\ 773.8409 & -0.6 & -0.8 & 10 . \\ 773.8382 & 2.1 & 2.7 & 11.5 \\ 773.8404 & -0.1 & -0.1 & 18 . \\ 773.8430 & -2.7 & -3.5 & 17.5 \\ 773.8401 & 0.2 & 0.3 & 8.5\end{array}$

i-FIT

-FIT (Norm) Formula

43.1
43.2
46.3
46.8
46.9
48.3

0.7
0.8
3.9
4.4
4.5
5.9

\begin{tabular}{llll|l}
$\mathrm{C} 22$ & $\mathrm{H} 20$ & $\mathrm{~N} 9$ & 03 & $\mathrm{Br} 4$ \\
$\mathrm{C} 21$ & $\mathrm{H} 24$ & $\mathrm{~N} 5$ & 07 & $\mathrm{Br} 4$
\end{tabular}

$\begin{array}{lllll}\mathrm{C} 17 & \mathrm{H} 20 & \mathrm{~N} 11 & 05 & \mathrm{Br} 4 \\ \mathrm{Cr} & \mathrm{Br} & \mathrm{H} \\ \mathrm{H} 11 & \mathrm{~N} 13 & 0 & -\end{array}$

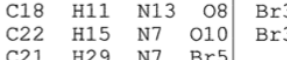


S19. ${ }^{1} \mathrm{H}$ NMR Spectrum of Slagenin D (3) in DMSO- $d_{6}$

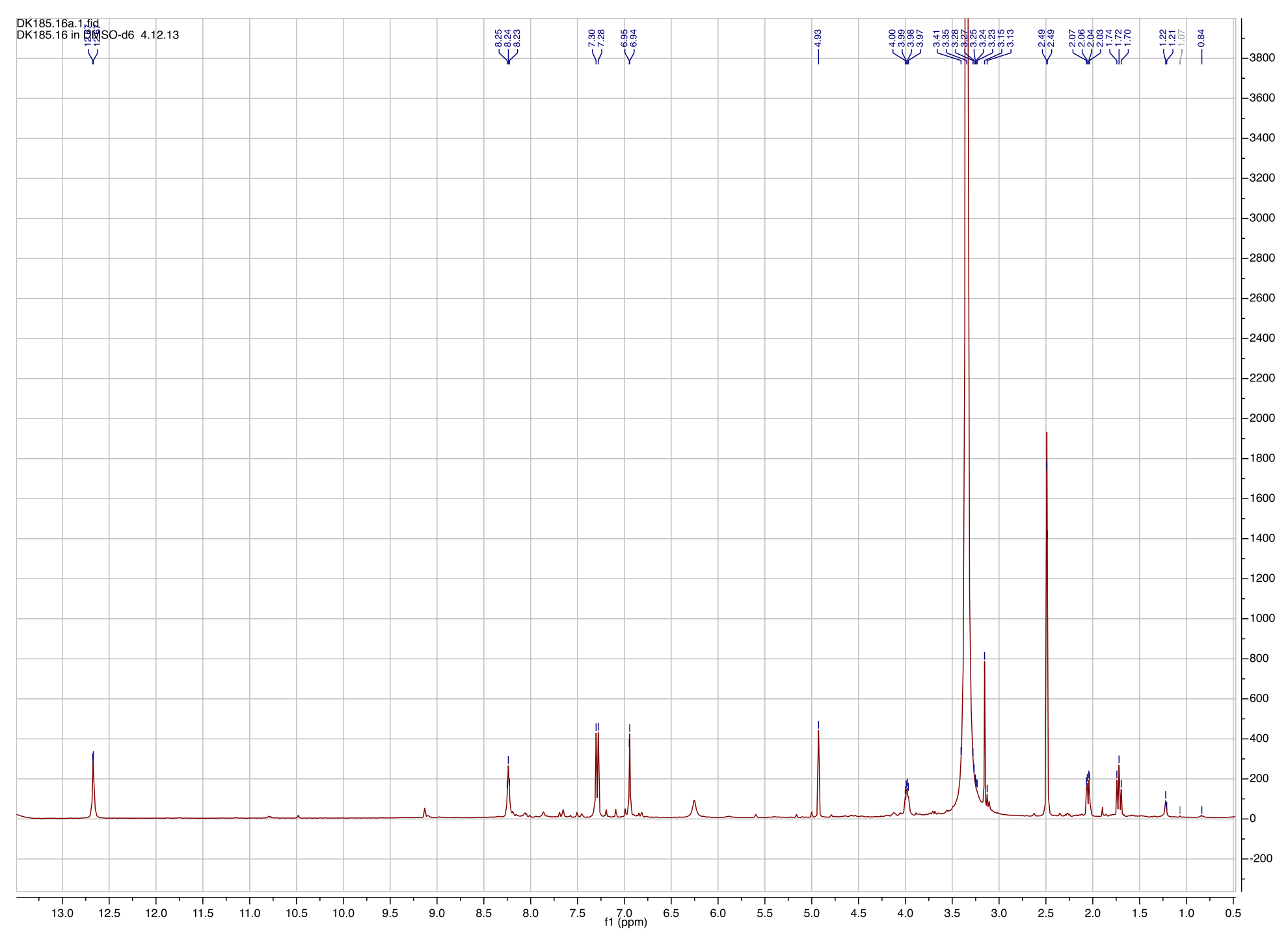


S20. ${ }^{13}$ C NMR Spectrum of Slagenin D (3) in DMSO- $d_{6}$

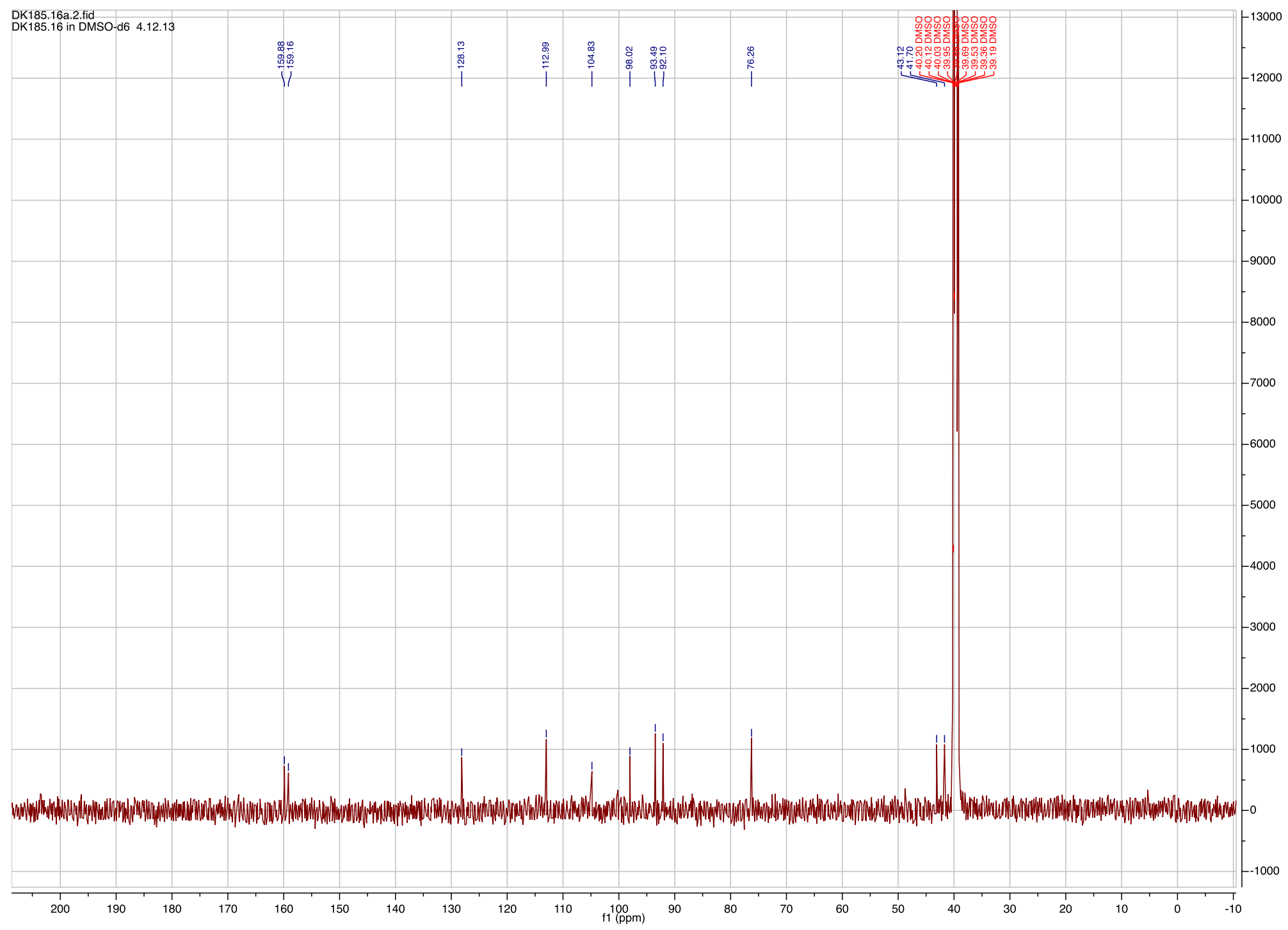


S21. HSQC Spectrum of Slagenin D (3) in DMSO- $d_{6}$

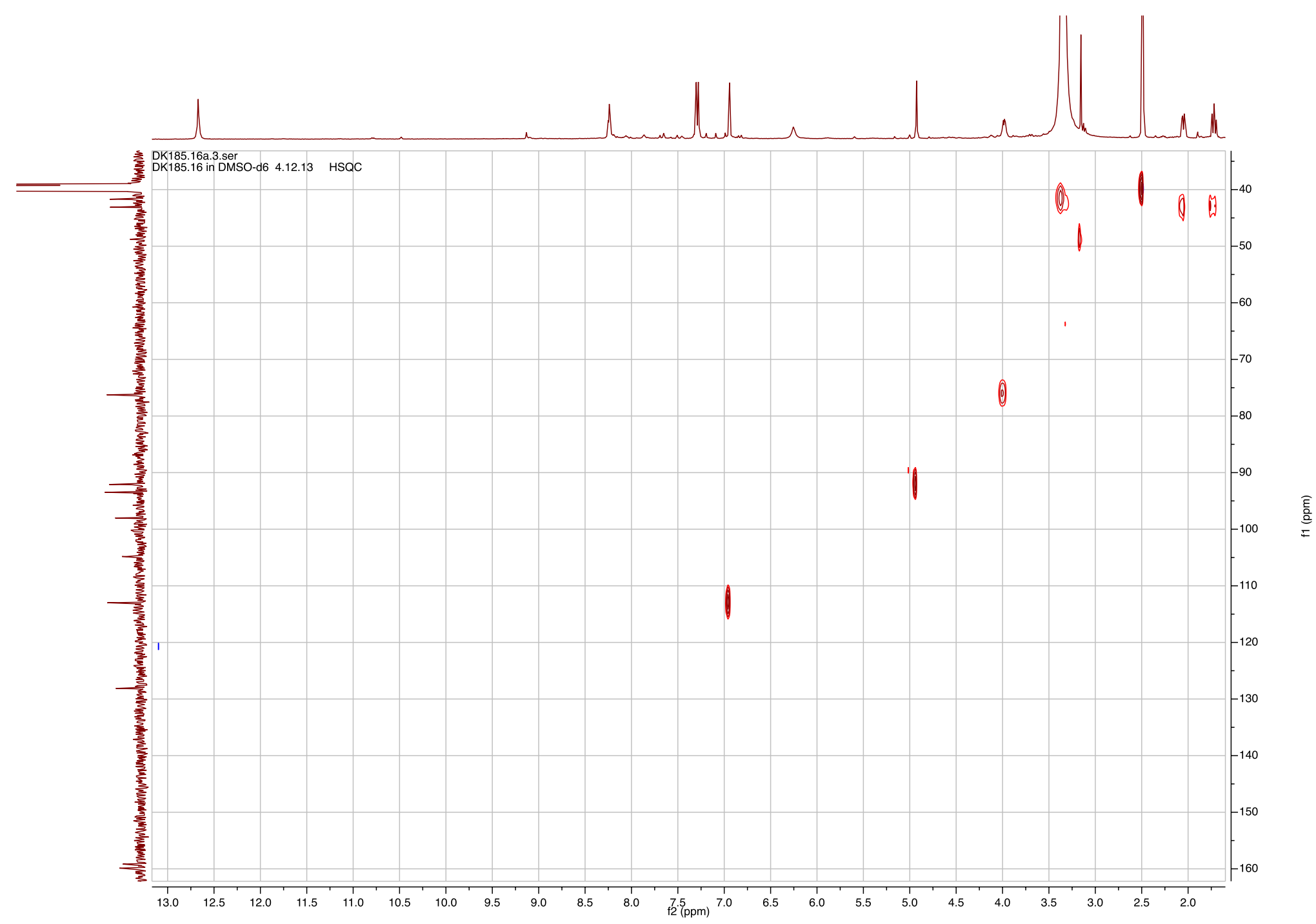


S22. HMBC Spectrum of Slagenin D (3) in DMSO- $d_{6}$

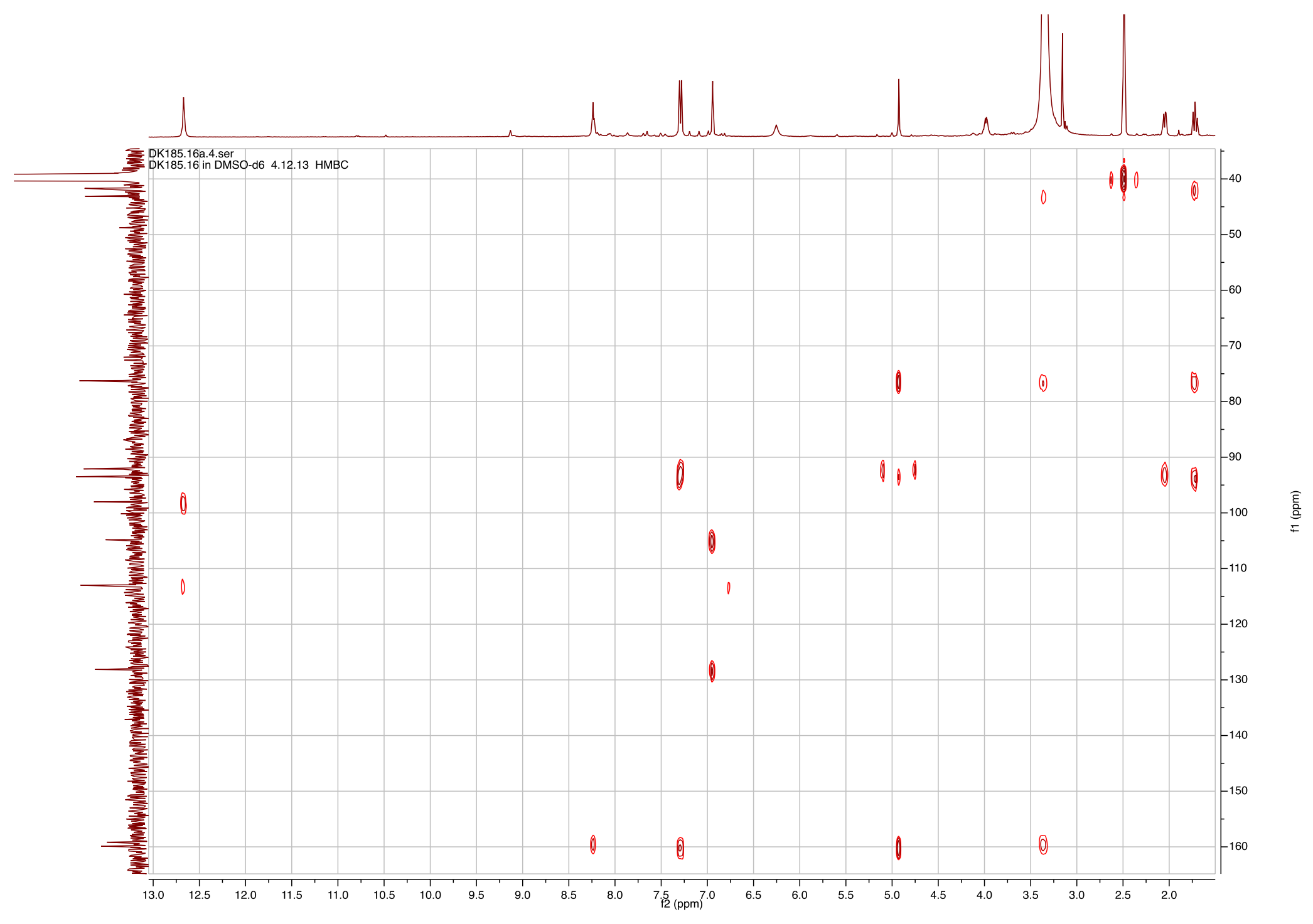


S23. COSY Spectrum of Slagenin D (3) in DMSO- $d_{6}$

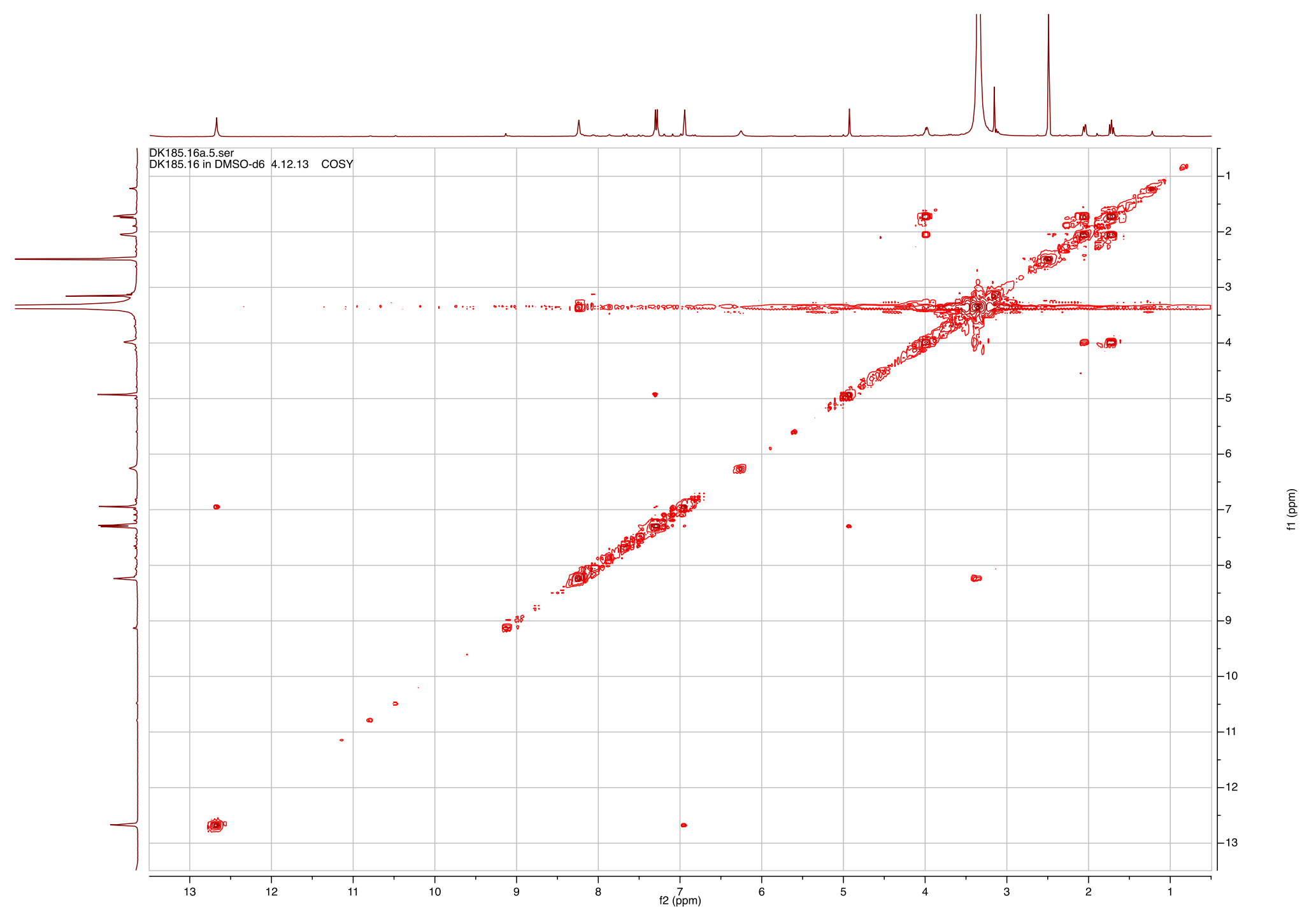


S24. ROESY Spectrum of Slagenin D (3) in DMSO- $d_{6}$

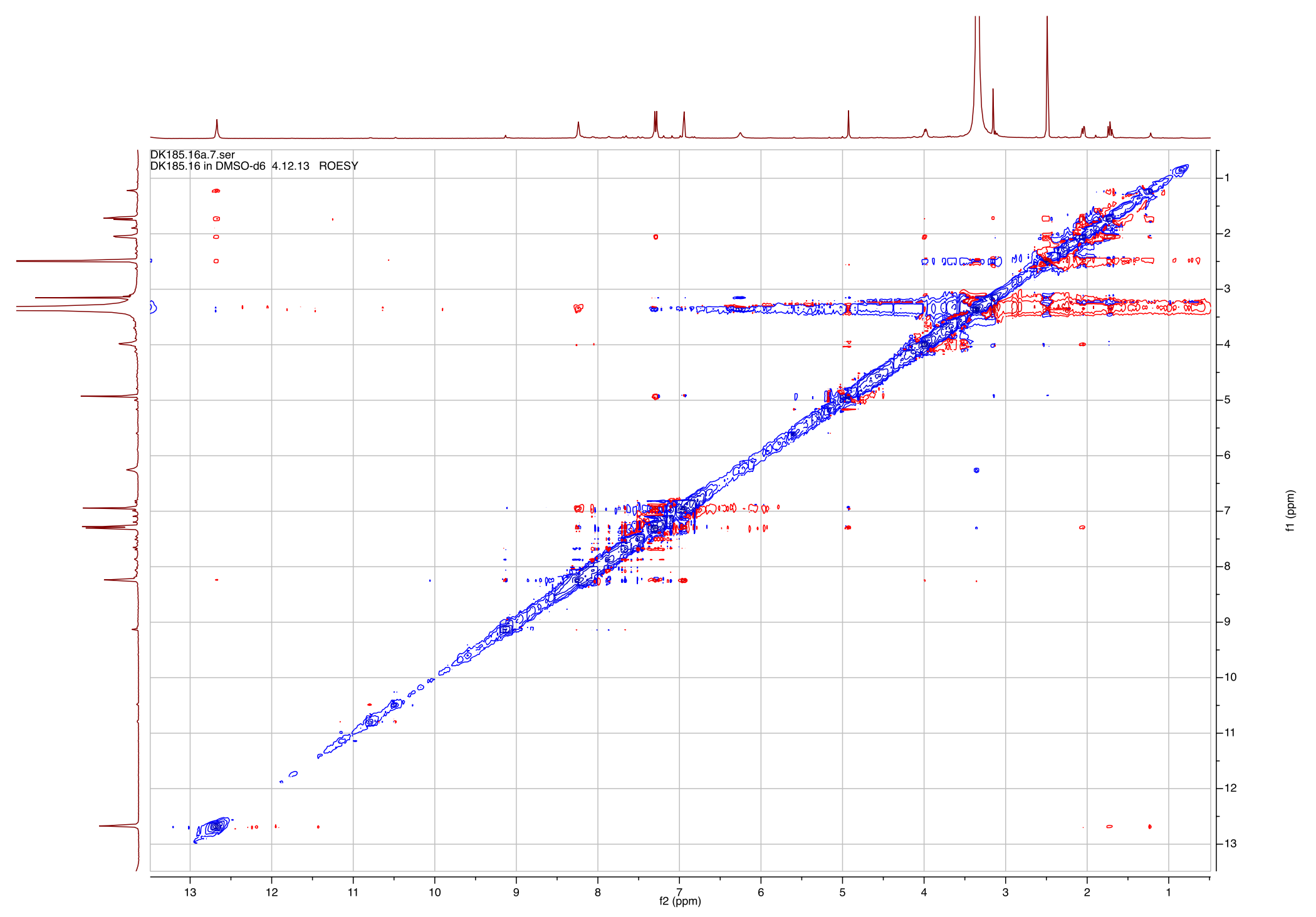


S25. Table S4. NMR data of slagenin D (3) in DMSO- $d_{6}{ }^{\mathrm{a}}$

\begin{tabular}{|c|c|c|c|c|c|}
\hline Position & $\delta_{\mathrm{C}}^{\mathrm{b}}$ & $\delta_{\mathrm{H}}$, mult., $J(\mathrm{~Hz})$ & $\delta_{\mathrm{H}}$, mult., $J(\mathrm{~Hz})^{\mathrm{c}}$ & $\begin{array}{l}\text { HMBC } \\
\text { correlations }\end{array}$ & NOE correlations \\
\hline 1 & & $12.67, \mathrm{~s}$ & $13.35, \mathrm{~s}$ & & 4,7 \\
\hline 2 & $104.8, \mathrm{C}$ & & & 4 & \\
\hline 3 & 98.0, C & & & 1 & \\
\hline 4 & 113.0, $\mathrm{CH}$ & $6.94, \mathrm{~s}$ & $7.22, \mathrm{~d}(2.5)$ & 1 & 1,7 \\
\hline 5 & 128.1, C & & & 4 & \\
\hline 6 & $159.2, \mathrm{C}$ & & & 7,8 & \\
\hline 7 & & $8.24, \mathrm{t}(5.8)$ & $8.73, \mathrm{brs}$ & & $1,4,8,9$ \\
\hline 8 & $41.7, \mathrm{CH}_{2}$ & $3.36, \mathrm{~m}$ & $3.50, \mathrm{~m}$ & $10 \mathrm{~b}$ & 7,9 \\
\hline 9 & $76.3, \mathrm{CH}$ & $\begin{array}{l}3.99, \mathrm{dtd} \quad(11.0 \\
4.9,4.5)\end{array}$ & $4.11, \mathrm{~m}$ & $8,10 b, 15$ & $7,8,10 \mathrm{a}^{\mathrm{d}}$ \\
\hline $10 \mathrm{a}$ & $43.2, \mathrm{CH}_{2}$ & $\begin{array}{l}2.05, \quad \mathrm{dd} \quad(11.0 \\
4.5)\end{array}$ & $2.19, \mathrm{dd}(12.0,3.9)$ & 8 & $7,9 \mathrm{~d}, 10 \mathrm{~b}, 12^{\mathrm{d}}$ \\
\hline $\mathrm{b}$ & & $1.72, \mathrm{t}(11.0)$ & $1.86, \mathrm{t}(12.0)$ & & $7,10 \mathrm{a}, 15,11-\mathrm{OH}^{\mathrm{c}}$ \\
\hline 11 & $93.5, \mathrm{C}$ & & & $10 \mathrm{a}, 10 \mathrm{~b}, 12,13$ & \\
\hline $11-\mathrm{OH}$ & & $6.25 \mathrm{brs}$ & $6.78, \mathrm{~s}$ & & $10 b^{d}, 12,15^{d}$ \\
\hline 12 & & $7.28, \mathrm{~s}$ & $8.07, \mathrm{~s}$ & & $10 \mathrm{a}^{\mathrm{d}}, 11-\mathrm{OH}$ \\
\hline 13 & 159.9, C & & & $12,14,15$ & \\
\hline 14 & & $7.30, \mathrm{~s}$ & $8.07, \mathrm{~s}$ & & 15 \\
\hline 15 & $92.1, \mathrm{CH}$ & $4.93, \mathrm{~s}$ & $5.15, \mathrm{~s}$ & 12 & $10 \mathrm{~b}, 14,11-\mathrm{OH}^{\mathrm{d}}$ \\
\hline
\end{tabular}

$\mathrm{a}_{500.13 \mathrm{MHz} \text { for }}{ }^{1} \mathrm{H}$ and $125.76 \mathrm{MHz}$ for ${ }^{13} \mathrm{C}$. ${ }^{\mathrm{b}}$ Multiplicity and assignment from $\mathrm{HSQC}$ experiment.

${ }^{\mathrm{c}}$ Measured in DMF- $d_{7}$ at $233^{\circ} \mathrm{K}$. dNOE's measured in DMF- $d_{7}$ at $233^{\circ} \mathrm{K}$. 
S26. Table S5. Comparison of the NMR Data $\left(500 \mathrm{MHz}, \mathrm{DMSO}-d_{6}\right)$ of Slagenin D (3), Slagenin A

\begin{tabular}{|c|c|c|c|c|}
\hline \multirow[b]{2}{*}{ position } & \multicolumn{2}{|r|}{ slagenin D (7) } & \multicolumn{2}{|c|}{ slagenin A } \\
\hline & $\delta_{\mathrm{C}}$, type & $\delta_{\mathrm{H}}(J$ in $\mathrm{Hz})$ & $\delta_{C}$, type & $\delta_{\mathrm{H}}(J$ in $\mathrm{Hz})$ \\
\hline 1 & & $12.67, \mathrm{~s}$ & & 11.81, brs \\
\hline 2 & 104.8, C & & 126.7, CH & $6.97, \mathrm{~s}$ \\
\hline 3 & $98.0, \mathrm{C}$ & & $94.9, \mathrm{C}$ & \\
\hline 4 & 113.0, $\mathrm{CH}$ & $6.94, \mathrm{~s}$ & $111.7, \mathrm{CH}$ & 6.87, brs \\
\hline 5 & $128.1, \mathrm{C}$ & & $126.7, \mathrm{C}$ & \\
\hline 6 & 159.2, C & & 159.7, C & \\
\hline 7 & & $8.24, \mathrm{t}(5.8)$ & & $8.21, \mathrm{t}(5.6)$ \\
\hline 8 & $41.7, \mathrm{CH} 2$ & $3.36, \mathrm{~m}$ & $41.5, \mathrm{CH} 2$ & $3.38, \mathrm{~m}$ \\
\hline 9 & $76.3, \mathrm{CH}$ & $3.99, \operatorname{dtd}(11.0,4.9,4.5)$ & $76.1, \mathrm{CH}$ & $4.00, \mathrm{~m}$ \\
\hline $10 \mathrm{a}$ & $43.2, \mathrm{CH} 2$ & $2.05, \mathrm{dd}(11.0,4.5)$ & $43.0, \mathrm{CH} 2$ & 2.06, dd $(11.6,3.6)$ \\
\hline $\mathrm{b}$ & & $1.72, \mathrm{t}(11.0)$ & & $1.73, \mathrm{t}(11.6)$ \\
\hline 11 & $93.5, \mathrm{C}$ & & $93.3, \mathrm{C}$ & \\
\hline $11-\mathrm{OH}$ & & $6.25 \mathrm{brs}$ & & \\
\hline 12 & & $7.28, \mathrm{~s}$ & & $7.28, \mathrm{~s}$ \\
\hline 13 & 159.9, C & & 159.7, C & \\
\hline 14 & & $7.30, \mathrm{~s}$ & & $7.30, \mathrm{~s}$ \\
\hline 15 & $92.1, \mathrm{CH}$ & $4.93, \mathrm{~s}$ & $91.9, \mathrm{CH}$ & 4.94, s \\
\hline
\end{tabular}


S27. HR ESI MS data of Slagenin D (3)

Elemental Composition Report

Page 1

Single Mass Analysis

Tolerance $=5.0 \mathrm{mDa}$ / DBE: $\min =-1.5, \max =50.0$

Number of isotope peaks used for i-FIT $=3$

Monoisotopic Mass, Even Electron lons
996 formula(e) evaluated with 13 results within limits (up to 4 closest results for each mass)

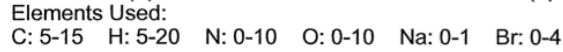

DK185.16
carmeli909 39 (1.736) $\mathrm{Cm}$ (37:42)

Dimitry Kovalerchik

46.9117

1: TOF MS ES+

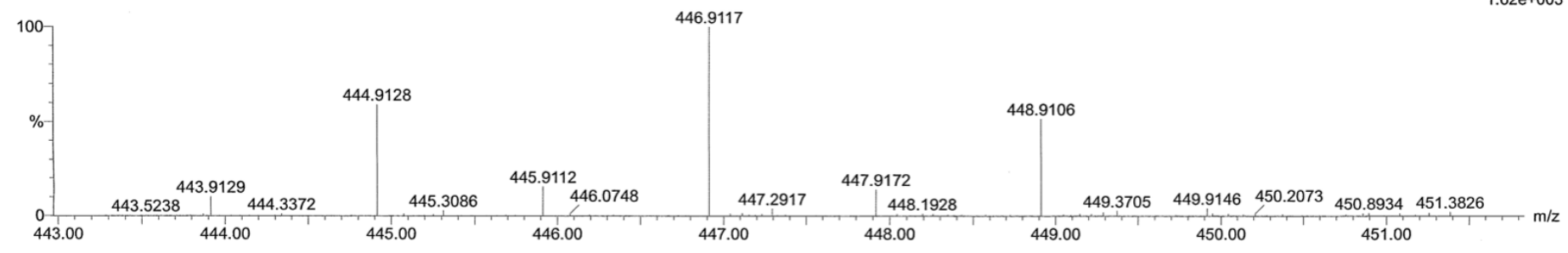

$\begin{array}{llll}\text { Minimum: } & & & \\ \text { Maximum: } & 5.0 & 10.0 & 50.5 \\ & & \end{array}$

Mass Calc. Mass mDa PPM DBE i-FIT i-FIT (Norm) Formula

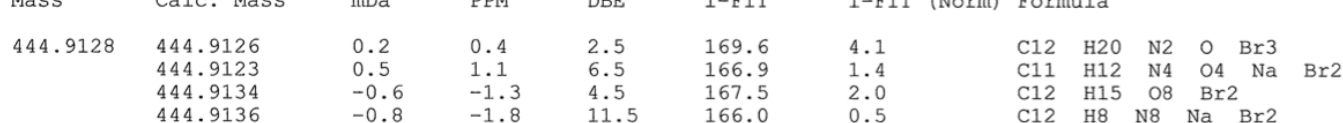


S28. ${ }^{1} \mathrm{H}$ NMR Spectrum of (-)-Monobromoagelaspongin (4) in DMSO- $d_{6}$

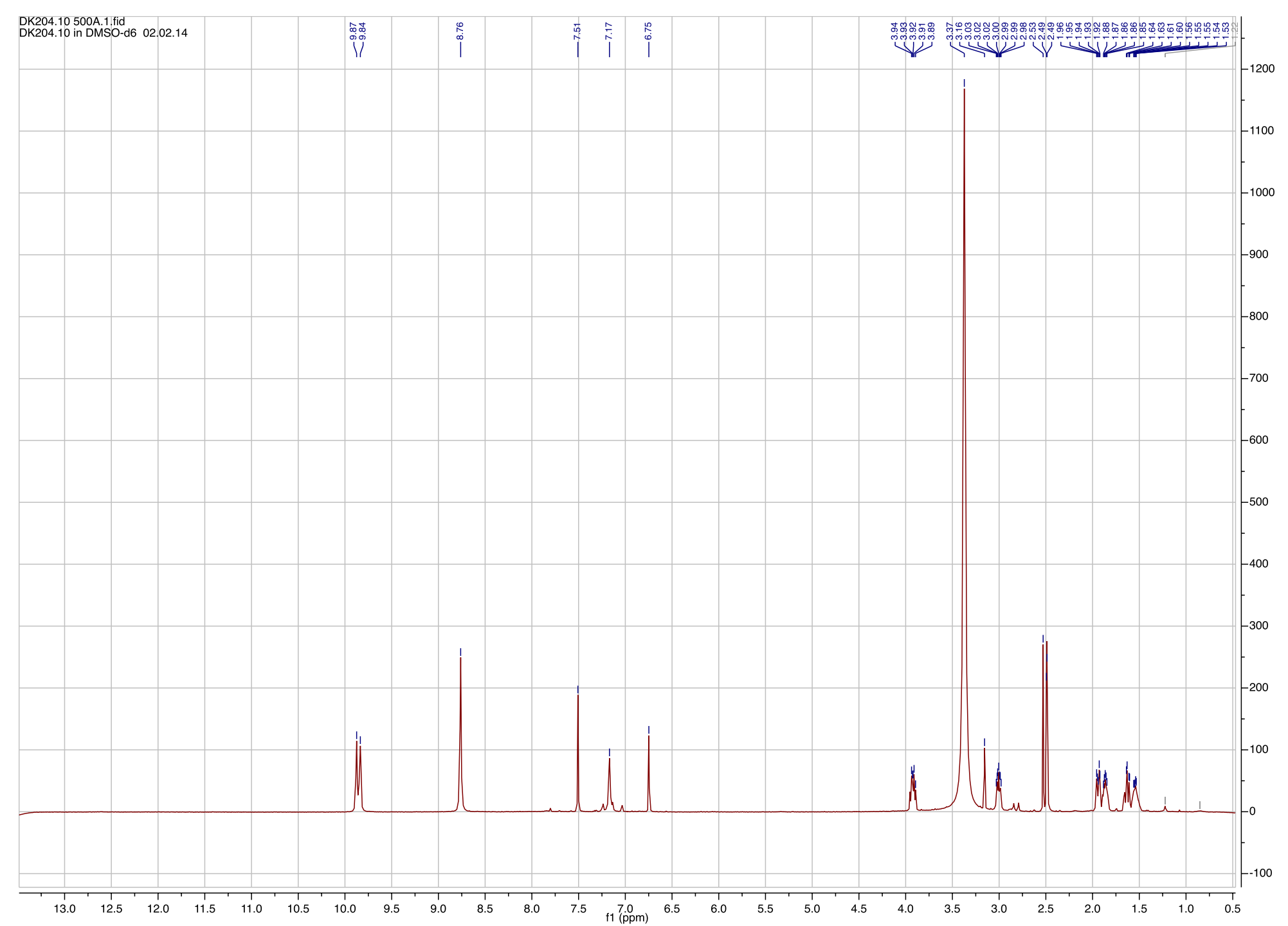


S29. ${ }^{13}$ C NMR Spectrum of (-)-Monobromoagelaspongin (4) in DMSO- $d_{6}$

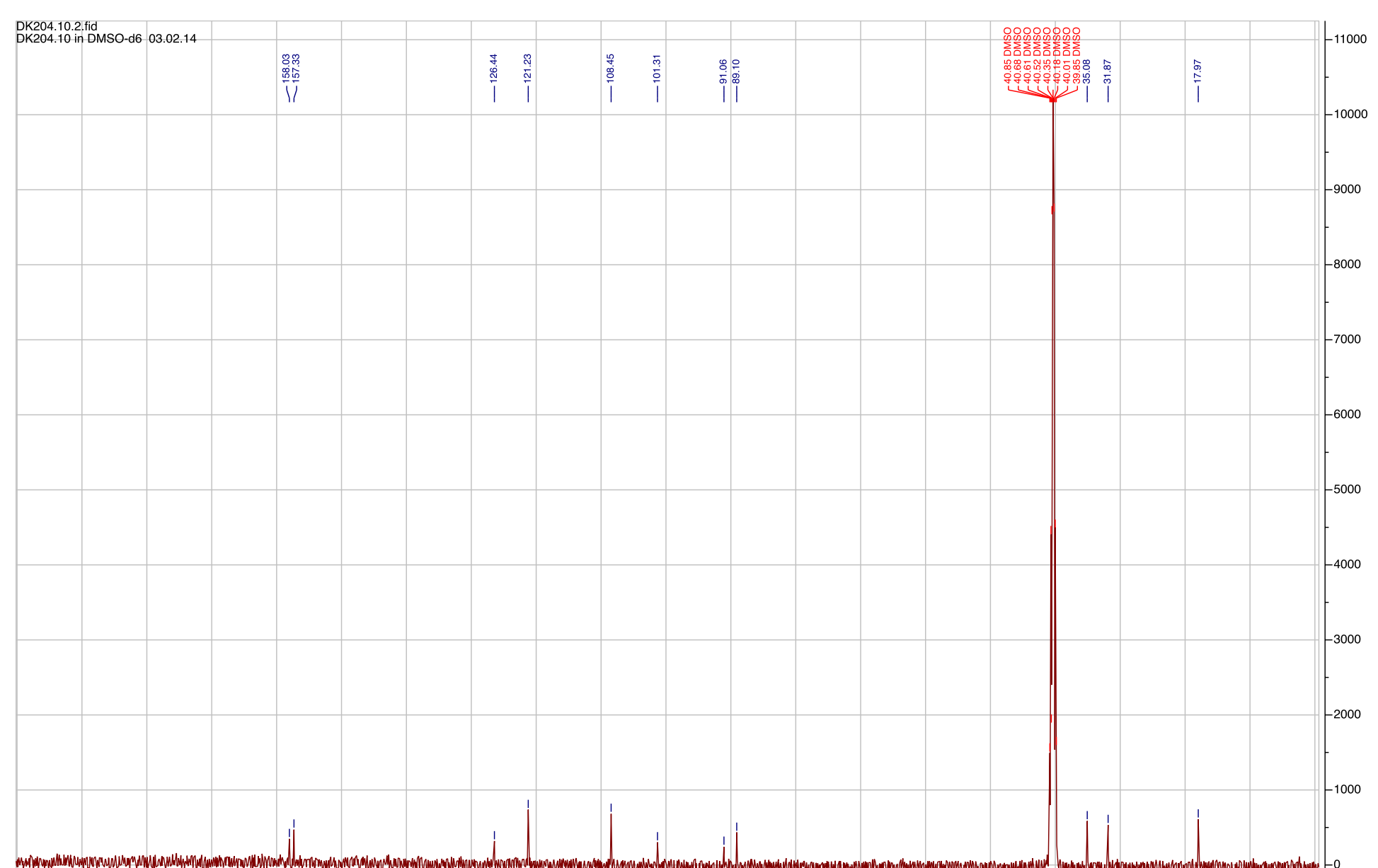

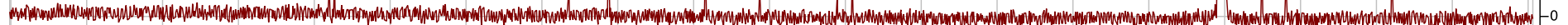

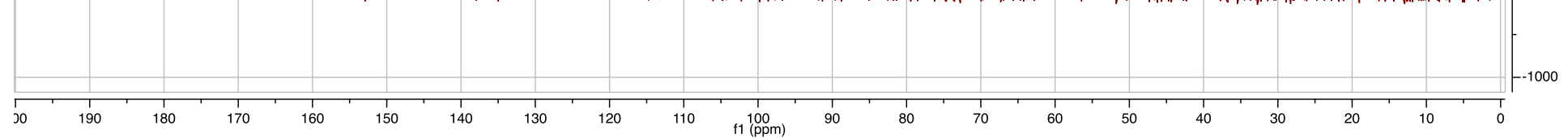


S30. HSQC Spectrum of (-)-Monobromoagelaspongin (4) in DMSO- $d_{6}$

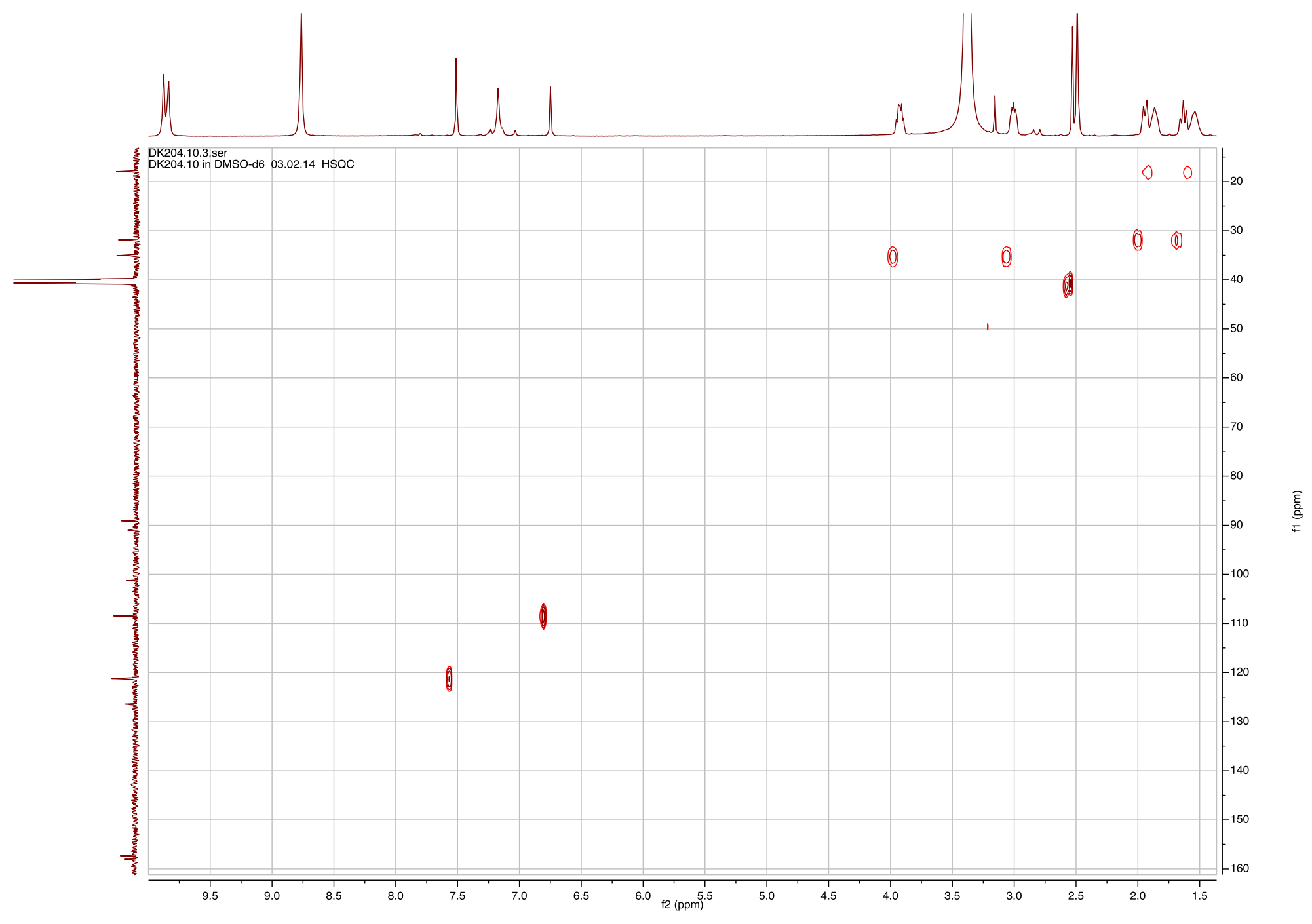


S31. HMBC Spectrum of (-)-Monobromoagelaspongin (4) in DMSO- $d_{6}$

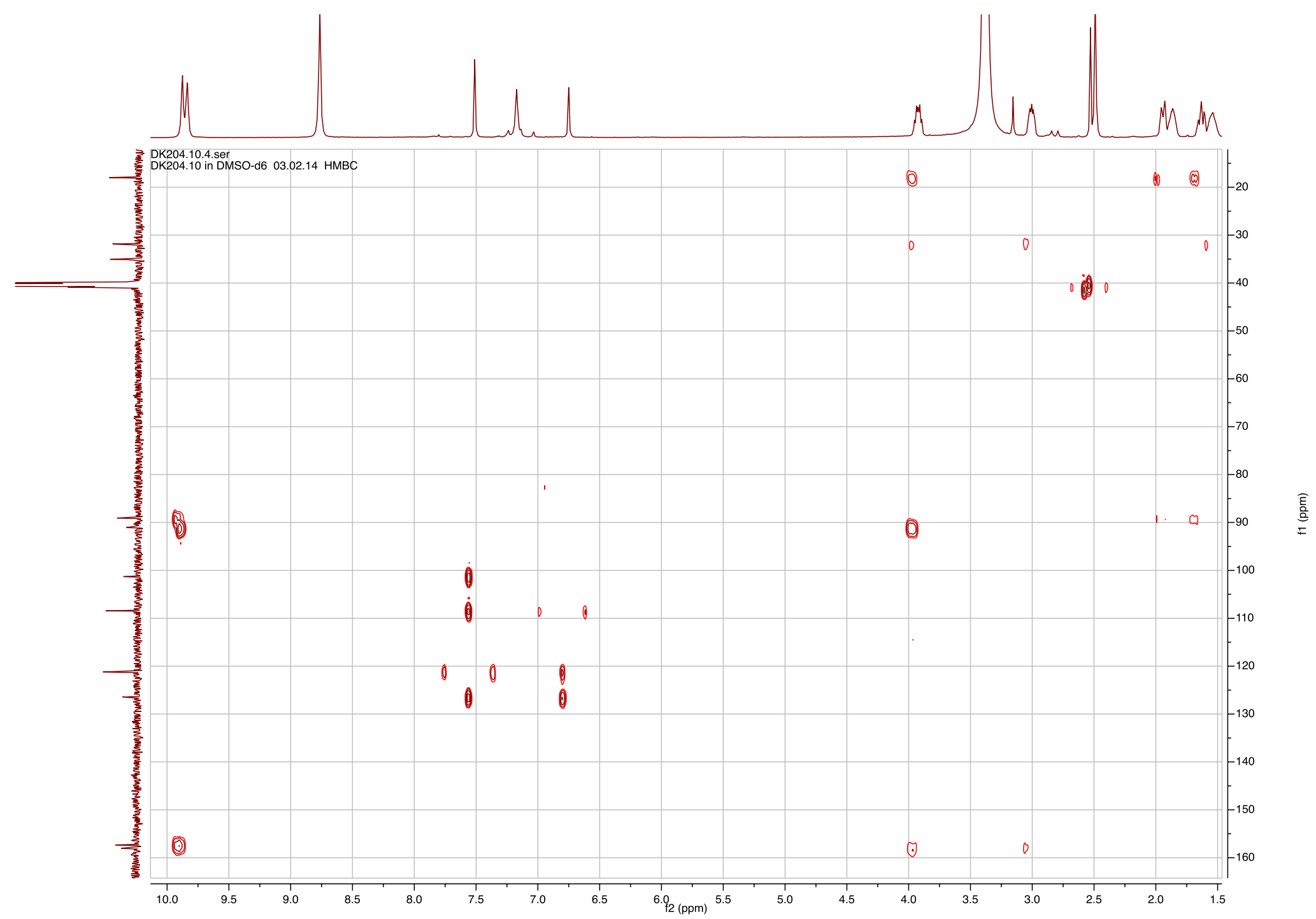


S32. COSY Spectrum of (-)-Monobromoagelaspongin (4) in DMSO- $d_{6}$

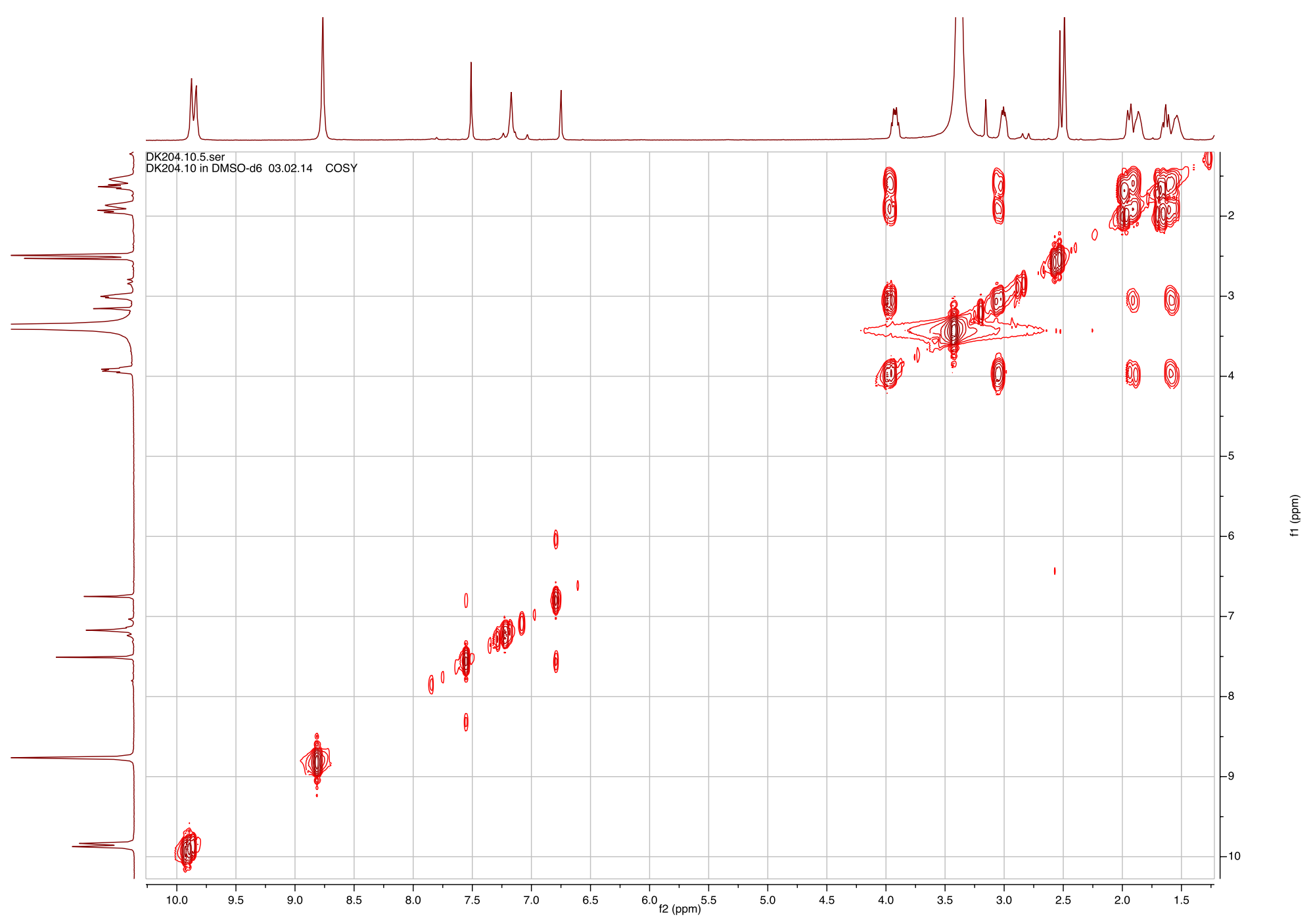


S33. ${ }^{1} \mathrm{H}$ NMR Spectrum of (-)-Monobromoagelaspongin (4) in DMF- $d_{7}$ at $233{ }^{\circ} \mathrm{K}$

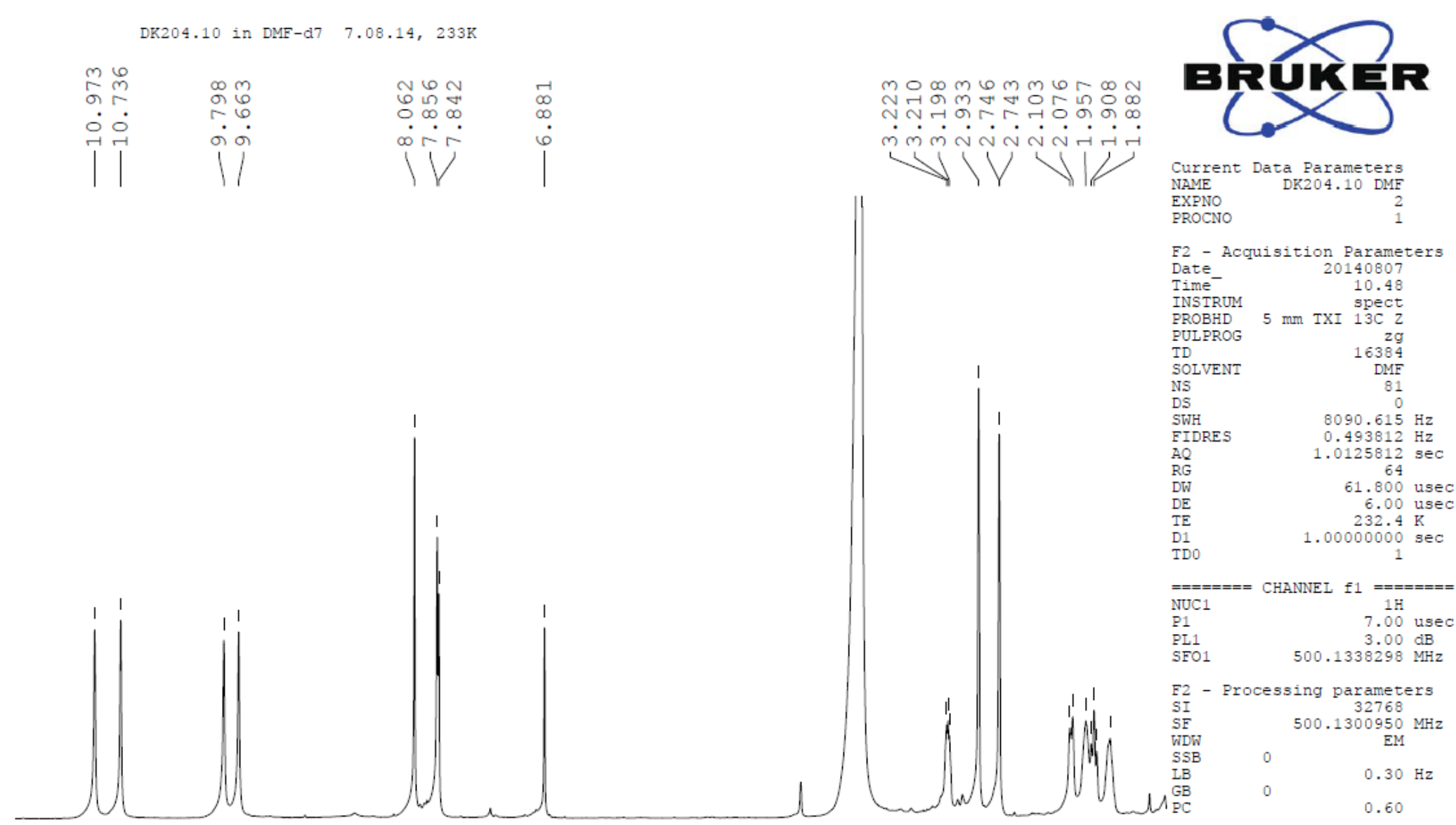

\begin{tabular}{|c|c|c|c|c|c|c|c|c|c|}
\hline 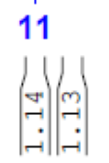 & 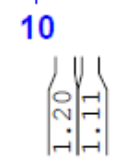 & 9 & 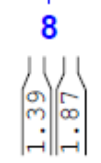 & $\begin{array}{l}7 \\
\left|\begin{array}{c}6 \\
0\end{array}\right|\end{array}$ & 6 & 5 & 4 & $\left|\begin{array}{l}3 \\
- \\
i\end{array}\right|$ & 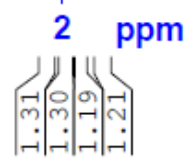 \\
\hline
\end{tabular}


S34. Difference-NOE Spectra of (-)-Monobromoagelaspongin (4) in DMF- $d_{7}$ at $233^{\circ} \mathrm{K}$

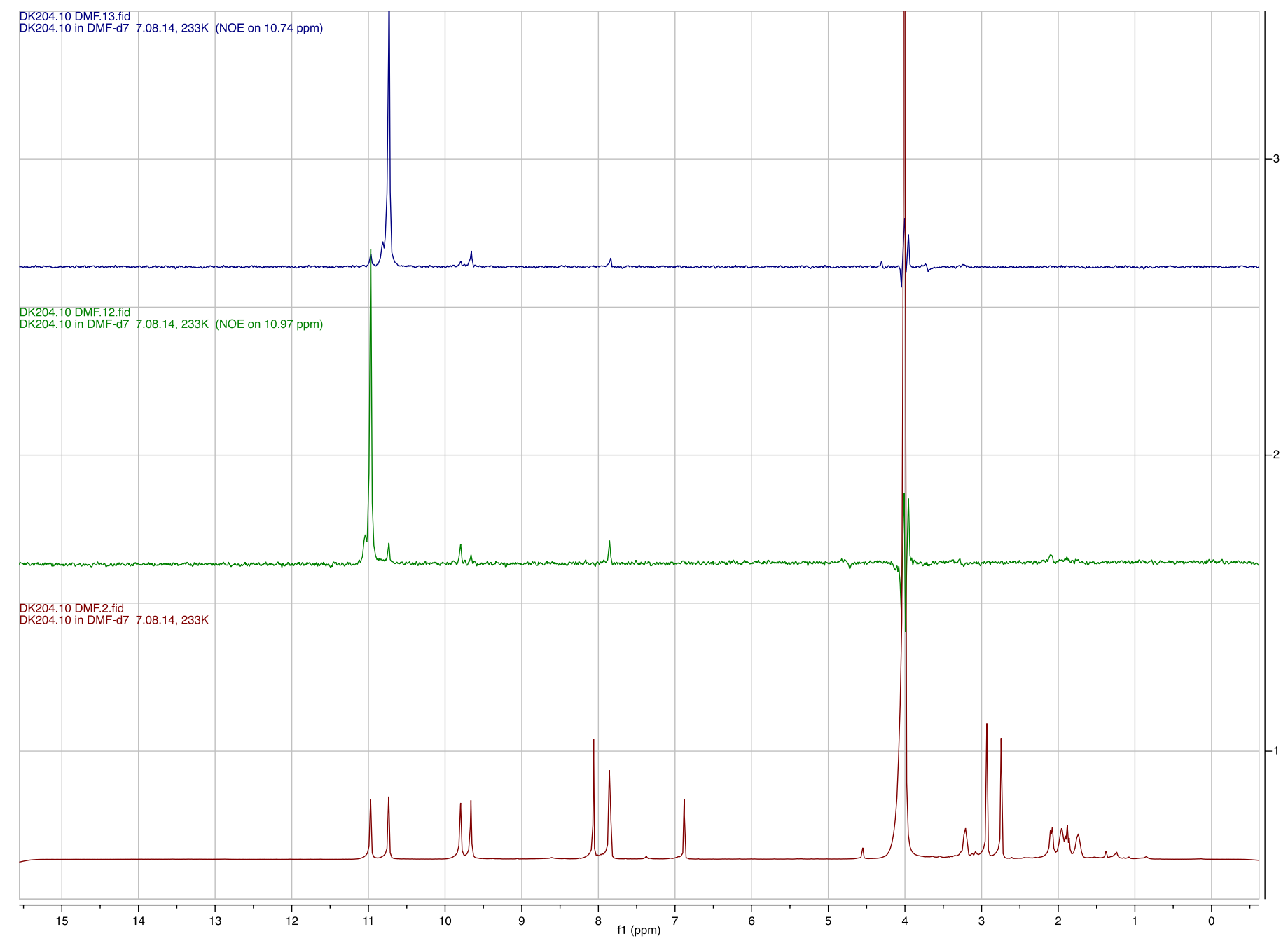


S35. Table S6. NMR Data of (-)-Monobromoagelaspongin (4) in DMSO- $d_{6}$ and in DMF- $d_{7}$ at $233{ }^{\circ} \mathrm{K}^{\mathrm{a}}$

\begin{tabular}{|c|c|c|c|c|c|}
\hline Position & $\delta_{\mathrm{C}}^{\mathrm{b}}$ & $\delta_{\mathrm{H}}$, mult., $J(\mathrm{~Hz})$ & $\delta_{\mathrm{H}}$, mult., $J(\mathrm{~Hz})^{\mathrm{c}}$ & HMBC correlations & NOE correlations ${ }^{d}$ \\
\hline 2 & $120.6, \mathrm{CH}$ & $7.51, \mathrm{~s}$ & $7.84, \mathrm{~s}$ & 4 & 4,14 \\
\hline 3 & 100.7, C & & & 2 & \\
\hline 4 & $107.8, \mathrm{CH}$ & $6.75, \mathrm{~s}$ & $6.88, \mathrm{~s}$ & 2 & 2 \\
\hline 5 & $125.8, \mathrm{C}$ & & & 2,4 & \\
\hline 6 & $157.8, \mathrm{C}$ & & & $8 \mathrm{a}, 8 \mathrm{~b}$ & \\
\hline $8 \mathrm{a}$ & $34.4, \mathrm{CH}_{2}$ & 3.92, ddd $(13.7,8.6,7.5)$ & $4.00, \mathrm{~m}$ & & $9 \mathrm{a}, 9 \mathrm{~b}, 10 \mathrm{~b}$ \\
\hline $\mathrm{b}$ & & 3.04, ddd $(13.7,7.6,5.0)$ & $3.21, \mathrm{dd}, 12.5,6.0$ & & $9 a, 9 b$ \\
\hline $9 \mathrm{a}$ & $17.3, \mathrm{CH}_{2}$ & $1.86, \mathrm{~m}$ & $1.96, \mathrm{~m}$ & $8 \mathrm{a}, 10 \mathrm{a}, 10 \mathrm{~b}$ & $8 \mathrm{a}, 8 \mathrm{~b}, 10 \mathrm{a}, 10 \mathrm{~b}$ \\
\hline $\mathrm{b}$ & & $1.54, \mathrm{~m}$ & $1.74, \mathrm{~m}$ & & $8 \mathrm{a}, 8 \mathrm{~b}, 10 \mathrm{a}, 10 \mathrm{~b}, 12$ \\
\hline $10 \mathrm{a}$ & $31.2, \mathrm{CH}_{2}$ & $1.94, \mathrm{dd}(13.0,3.2)$ & $2.09, \mathrm{~d}, 13.0$ & $8 \mathrm{a}, 8 \mathrm{~b}, 9 \mathrm{~b}, 11-\mathrm{OH}$ & $9 \mathrm{a}, 9 \mathrm{~b}, 11-\mathrm{OH}, 13-\mathrm{NH}_{2}$ \\
\hline $\mathrm{b}$ & & $1.63, \operatorname{td}(13.0,3.0)$ & $1.88, \mathrm{t}, 13.0$ & & $8 \mathrm{a}, 9 \mathrm{a}, 9 \mathrm{~b}, 11-\mathrm{OH}$ \\
\hline 11 & $88.5, \mathrm{C}$ & & & $10 \mathrm{~b}, 11-\mathrm{OH}, 12,14$ & \\
\hline $11-\mathrm{OH}$ & & $7.17, \mathrm{~s}$ & $7.86, \mathrm{~s}$ & & $10 \mathrm{a}, 10 \mathrm{~b}, 12$ \\
\hline 12 & & $9.88, \mathrm{~s}$ & 10.97, s & & $9 \mathrm{a}, 9 \mathrm{~b}, 11-\mathrm{OH}$ \\
\hline 13 & 156.7, C & & & $12,13-\mathrm{NH}_{2}, 14$ & \\
\hline $13-\mathrm{NH}_{2}$ & & $8.77, \mathrm{~s}$ & $9.80, \mathrm{~s}, 9.66, \mathrm{~s}$ & & $10 \mathrm{a}$ \\
\hline 14 & & $9.84, \mathrm{~s}$ & $10.74, \mathrm{~s}$ & & $2,13-\mathrm{NH}_{2}$ \\
\hline 15 & $90.4, \mathrm{C}$ & & & $8 \mathrm{a}, 11-\mathrm{OH}, 12,14$ & \\
\hline
\end{tabular}

${ }^{\mathrm{a}} 500.13 \mathrm{MHz}$ for ${ }^{1} \mathrm{H}$ and $125.76 \mathrm{MHz}$ for ${ }^{13} \mathrm{C}$. ${ }^{\mathrm{b}}$ Multiplicity and assignment from HSQC experiment.

${ }^{\mathrm{c}}$ Measured in DMF- $d_{7}$ at $233{ }^{\circ} \mathrm{K}$. d NOE's measured in DMF- $d_{7}$ at $233{ }^{\circ} \mathrm{K}$. 
S36. HR ESI MS data of (-)-Monobromoagelaspongin (4)

Elemental Composition Report

Page 1

Single Mass Analysis

Tolerance $=5.0 \mathrm{mDa}$ / DBE: $\min =-1.5, \max =50.0$

Number of isotope peaks used for $\mathrm{i}-\mathrm{FIT}=3$

Monoisotopic Mass, Even Electron lons

347 formula(e) evaluated with 6 results within limits (up to 4 best isotopic matches for each mass)

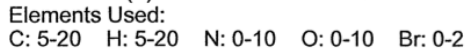

DK204.10

carmeligo1 71 (3.139) Cm (71:72)

Dimitry Kovalerchik

100

\%

312.0383

326.0256

328.0248

1: TOF MS ES+ 5 -

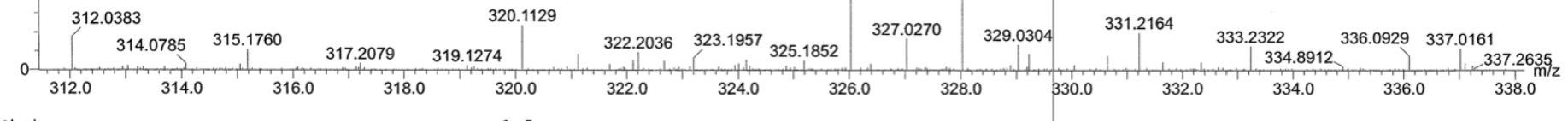

$\begin{array}{lll}5.0 & 10.0 & -1.5 \\ 50.0\end{array}$

Mass

Calc. Mass

$326.0256 \quad 326.0253$
326.0293
326.0239

326.0293
326.0239
326.0212

$\begin{array}{llll}0.3 & 0.9 & 7.5 & 101.6 \\ -3.7 & -11.3 & 11.5 & 102.8 \\ 1.7 & 5.2 & 2.5 & 103.6 \\ 4.4 & 13.5 & 3.5 & 106.4\end{array}$

i-FIT (Norm) Formula

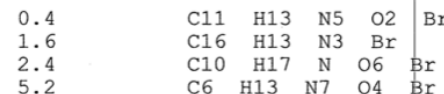


S37. ${ }^{1} \mathrm{H}$ NMR Spectrum of (-)-11-Deoxymonobromoagelaspongin (5) in DMSO- $d_{6}$

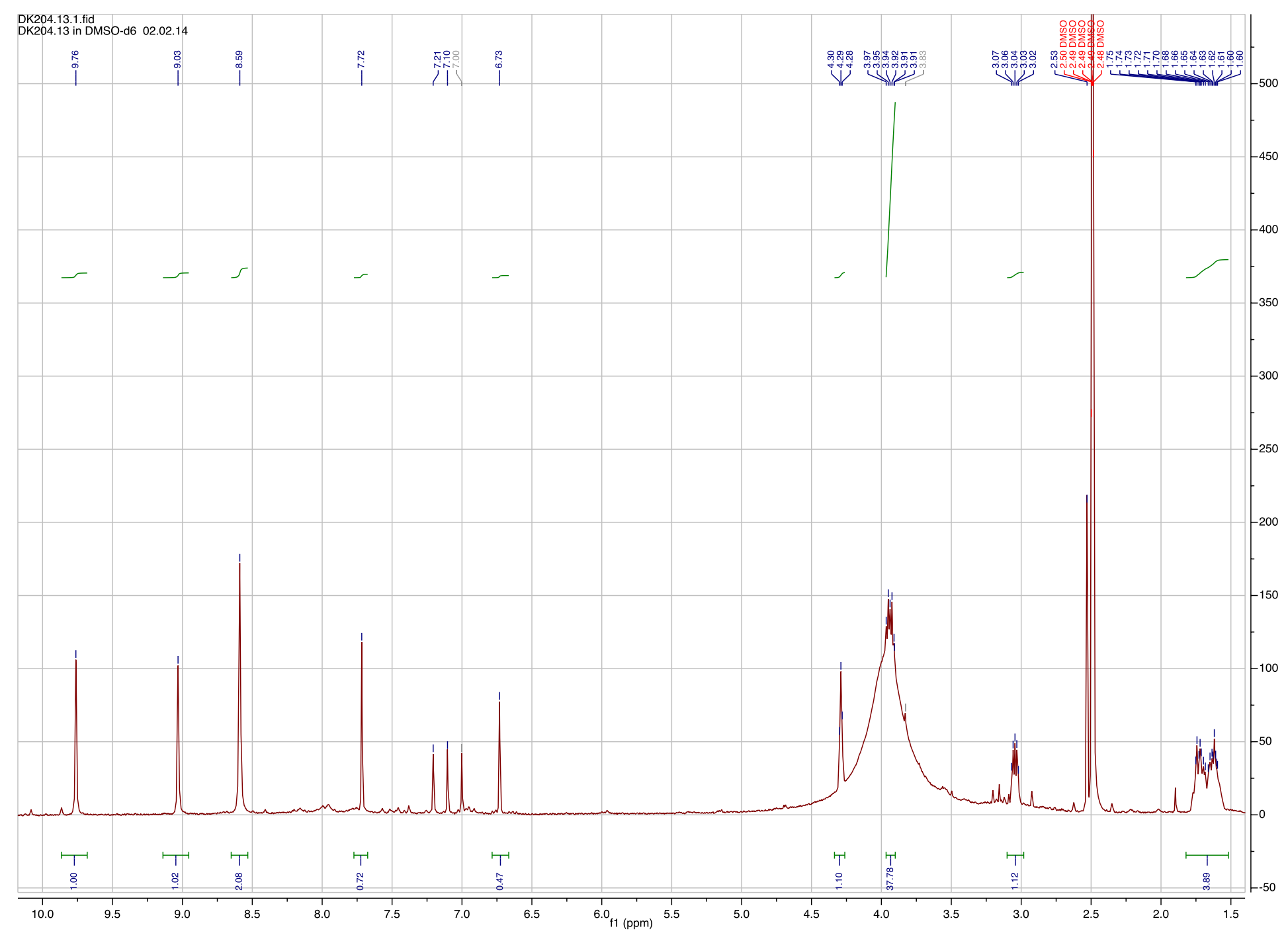


S38. ${ }^{13}$ C NMR Spectrum of (-)-11-Deoxymonobromoagelaspongin (5) in DMSO- $d_{6}$

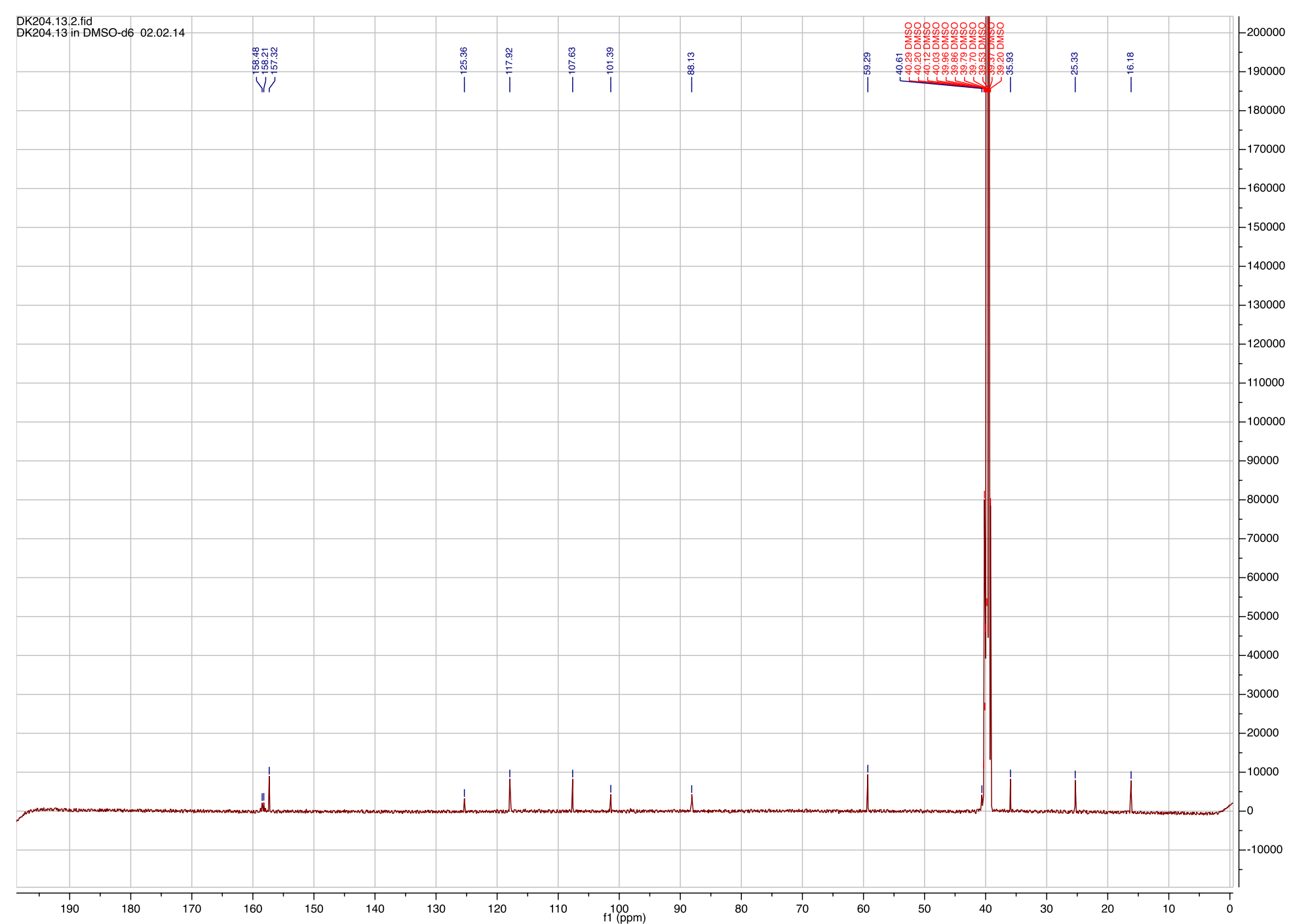


S39. HSQC Spectrum of (-)-11-Deoxymonobromoagelaspongin (5) in DMSO- $d_{6}$

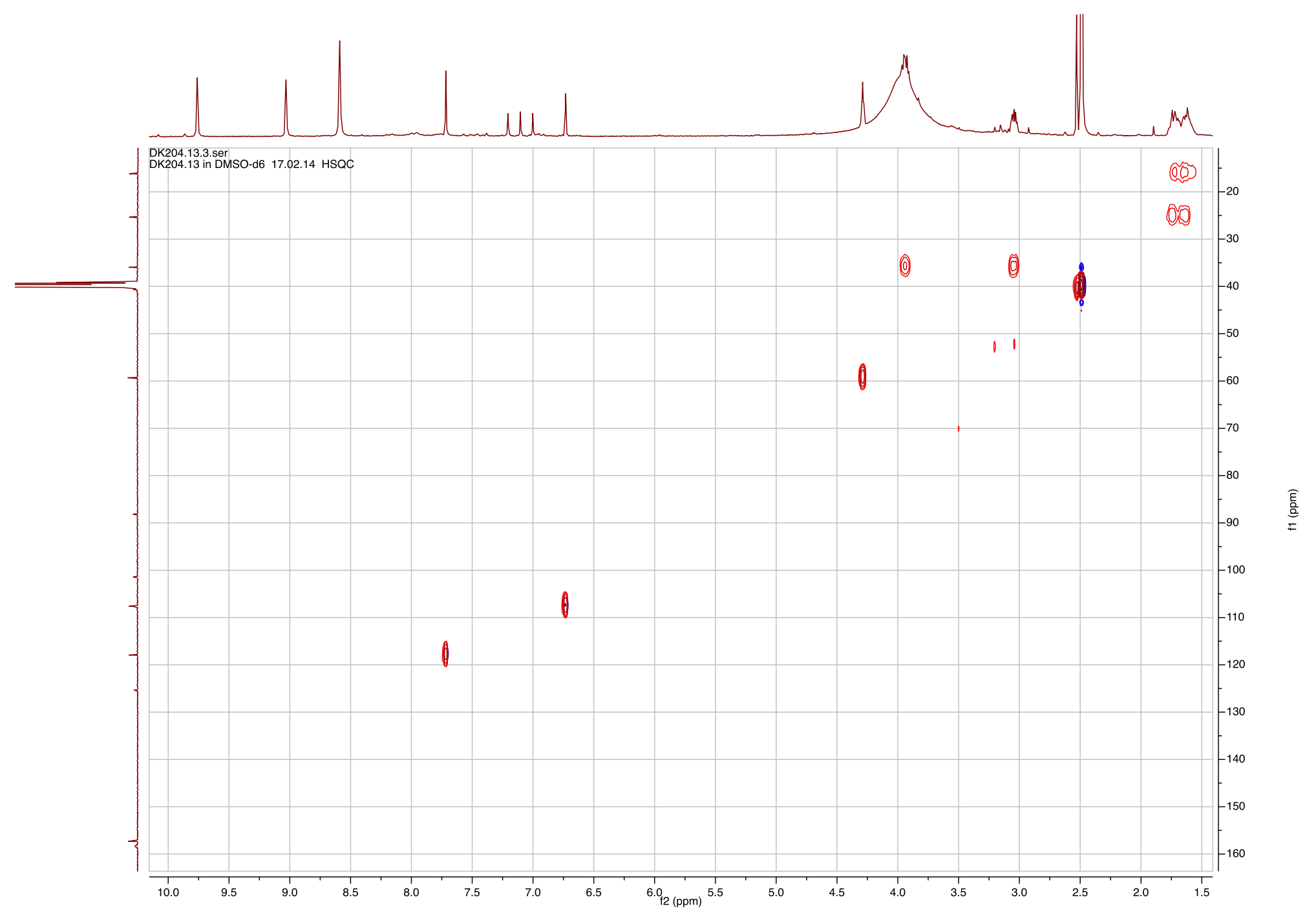


S40. HMBC Spectrum of (-)-11-Deoxymonobromoagelaspongin (5) in DMSO- $d_{6}$

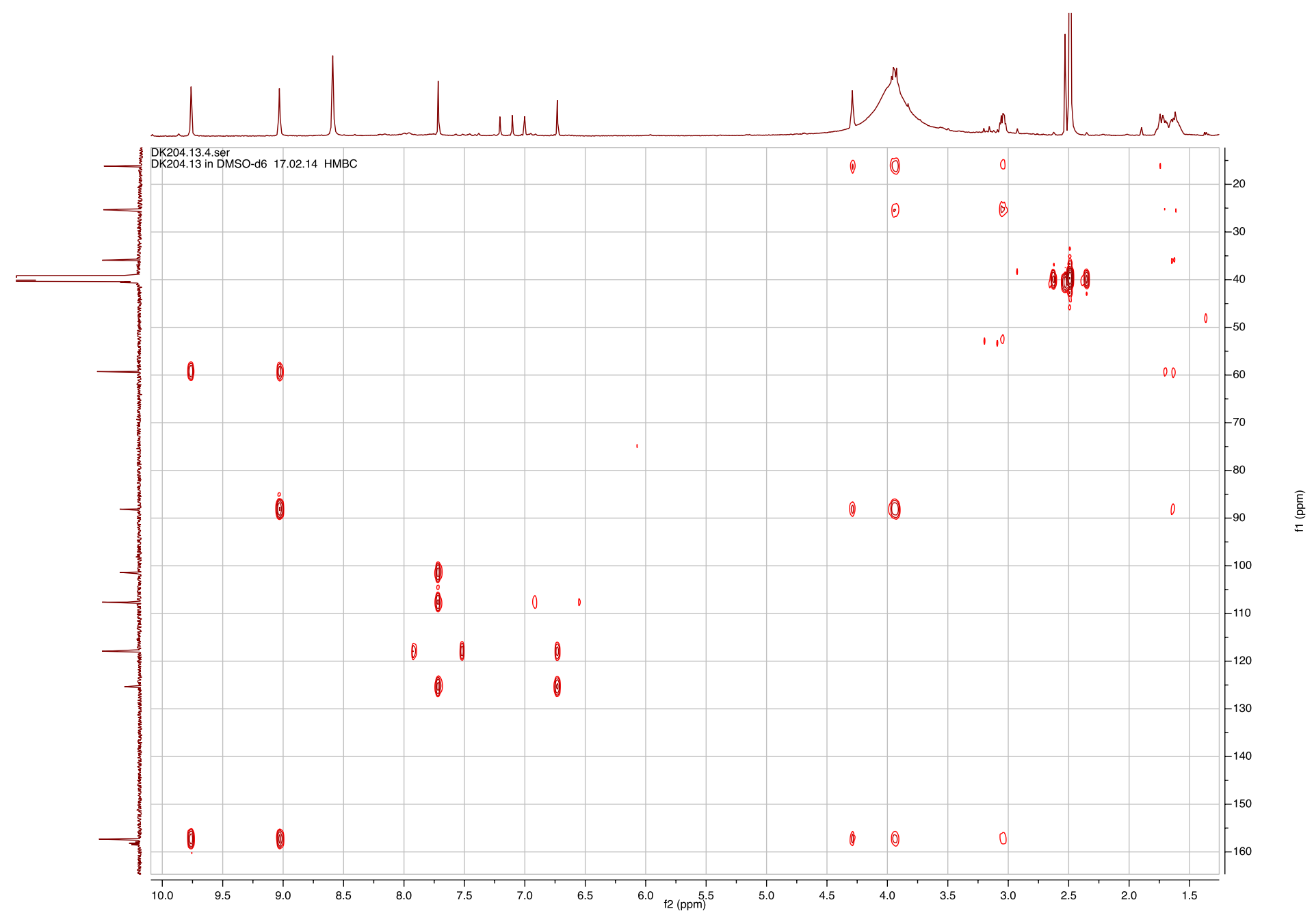


S41. COSY Spectrum of (-)-11-Deoxymonobromoagelaspongin (5) in DMSO- $d_{6}$

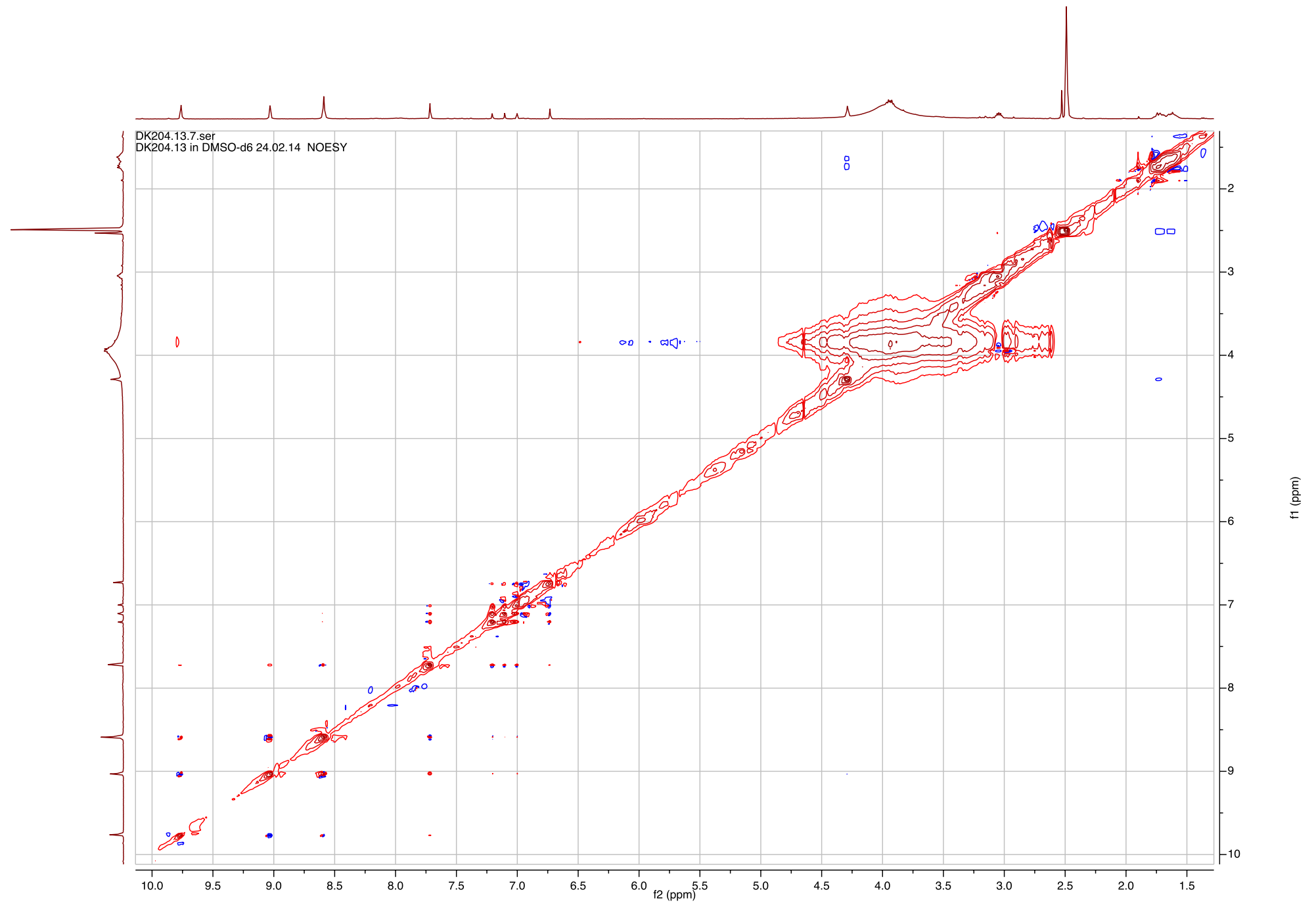


S42. Table S7. NMR Data of (-)-11-Deoxymonobromoagelaspongin (5) in DMSO- $d_{6}{ }^{\mathrm{a}}$

\begin{tabular}{lllll}
\hline Position & $\delta_{\mathrm{C}} \mathrm{b}^{2}$ & $\delta_{\mathrm{H}}$, mult., $J(\mathrm{~Hz})$ & HMBC correlations & NOE correlations ${ }^{\mathrm{d}}$ \\
\hline 2 & $118.6, \mathrm{CH}$ & $7.72, \mathrm{~d}(1.0)$ & 4 & \\
3 & $101.5, \mathrm{C}$ & & 2 & \\
4 & $107.7, \mathrm{CH}$ & $6.73, \mathrm{~d}(1.0)$ & 2 & \\
5 & $125.4, \mathrm{C}$ & & 2,4 & \\
6 & $157.4, \mathrm{C}$ & & $8 \mathrm{a}, 8 \mathrm{~b}$ \\
$8 \mathrm{a}$ & $36.0, \mathrm{CH}_{2}$ & $3.94, \mathrm{dt}(13.0,7.3)$ & & $9 \mathrm{~b}$ \\
$\mathrm{~b}$ & & $3.05, \mathrm{ddd}(13.0,6.0$, & \\
& & $4.5)$ & $8 \mathrm{a}$ \\
$9 \mathrm{a}$ & $16.2, \mathrm{CH}_{2}$ & $1.73, \mathrm{~m}$ & $8 \mathrm{~b}$ \\
$\mathrm{~b}$ & & $1.61, \mathrm{~m}$ & 12 \\
$10 \mathrm{a}$ & $25.4, \mathrm{CH} 2$ & $1.76, \mathrm{~m}$ & $10 \mathrm{a}, 12$ \\
$\mathrm{~b}$ & & $1.64, \mathrm{~m}$ & $8 \mathrm{a}, 8 \mathrm{~b}$ & $10 \mathrm{a}, 11$ \\
11 & $59.4, \mathrm{CH}$ & $4.29, \mathrm{td}(4.2,2.1)$ & 12,14 & \\
12 & & $9.03, \mathrm{~s}$ & & \\
13 & $157.4, \mathrm{C}$ & & $11,12,14$ & \\
$13-\mathrm{NH}_{2}$ & & $8.59, \mathrm{~s}$ & & \\
14 & & $9.76, \mathrm{~s}$ & & \\
15 & $88.2, \mathrm{C}$ & & $8 \mathrm{a}, 11,12$ & \\
\hline
\end{tabular}

${ }^{\mathrm{a}} 500.13 \mathrm{MHz}$ for ${ }^{1} \mathrm{H}$ and $125.76 \mathrm{MHz}$ for ${ }^{13} \mathrm{C} .{ }^{\mathrm{b}}$ Multiplicity and assignment from HSQC experiment. ${ }^{\mathrm{c}}$ Measured in DMF- $d_{7}$ at $233^{\circ} \mathrm{K}$. ${ }^{\mathrm{d}} \mathrm{NOE}$ 's measured in DMF- $d_{7}$ at $233^{\circ} \mathrm{K}$. 
S43. HR ESI MS data of (-)-11-Deoxymonobromoagelaspongin (5)

Elemental Composition Report

Page 1

Single Mass Analysis

Tolerance $=5.0 \mathrm{mDa}$ / DBE: $\min =-1.5, \max =50.0$

Number of isotope peaks used for i-FIT $=3$

Monoisotopic Mass, Even Electron lons

列

Elements Used:
C: $5-20 \quad$ H: $5-20 \quad$ N: 0-10 $\quad$ O: 0-10 Br: 0-2

DK204.13

Dimitry Kovalerchik

(6.592) Cm (150:155)

100

$310.0302 \quad 312.0292$

TOF MS ES+

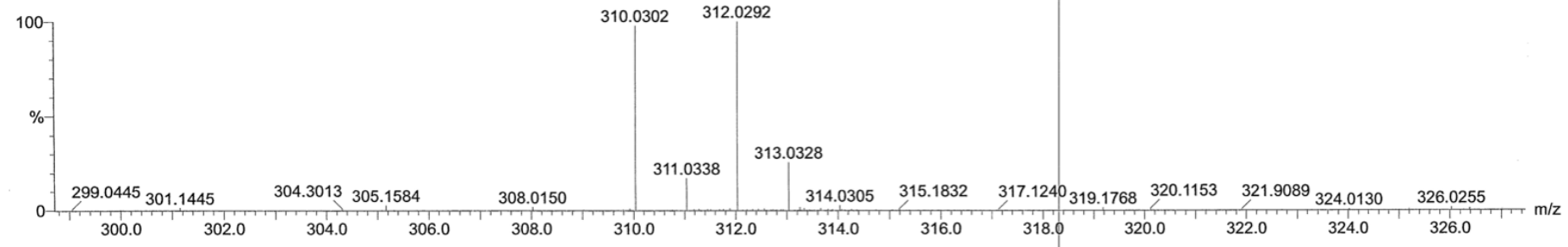

Minimum:

Mass Calc. Mass mDa PPM DBE

$\begin{array}{ll}310.0302 & 310.0303 \\ 310.029\end{array}$

310.0290
310.0263

$\begin{array}{llll}-0.1 & -0.3 & 7.5 & 410.6 \\ 1.2 & 3.9 & 2.5 & 412.6 \\ 3.9 & 12.6 & 3.5 & 416.8 \\ 3.1 & 10.0 & 5.5 & 428.6\end{array}$

i-FIT (Norm) Formul

$\begin{array}{llllll}0.1 & \text { C11 } & \text { H13 } & \text { N5 } & \text { O } & \text { Br } \\ 2.1 & \text { C10 } & \text { H17 } & \text { N } & \text { O5 } & \text { Br } \\ 6.3 & \text { C6 } & \text { H13 } & \text { N7 } & \text { O3 } & \text { Br } \\ 18.1 & \text { C6 } & \text { H8 } & \text { N5 } & \text { O10 } & \text { O10 }\end{array}$ 
S44. ${ }^{1} \mathrm{H}$ NMR Spectrum of (-)-11-O-Methylmonobromoagelaspongin (6) in DMSO- $d_{6}$

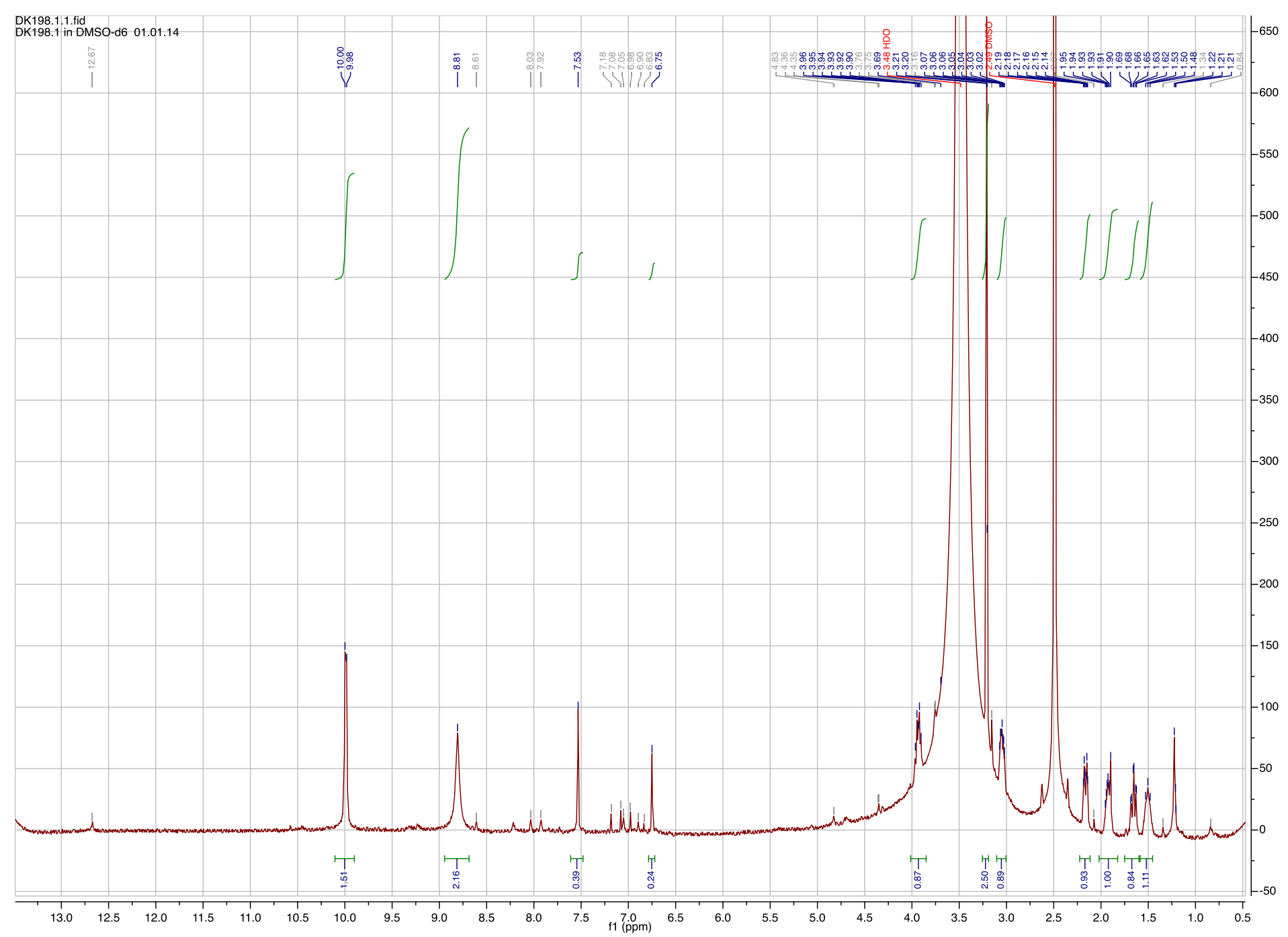


S45. ${ }^{13} \mathrm{C}$ NMR Spectrum of (-)-11-O-Methylmonobromoagelaspongin (6) in DMSO- $d_{6}$

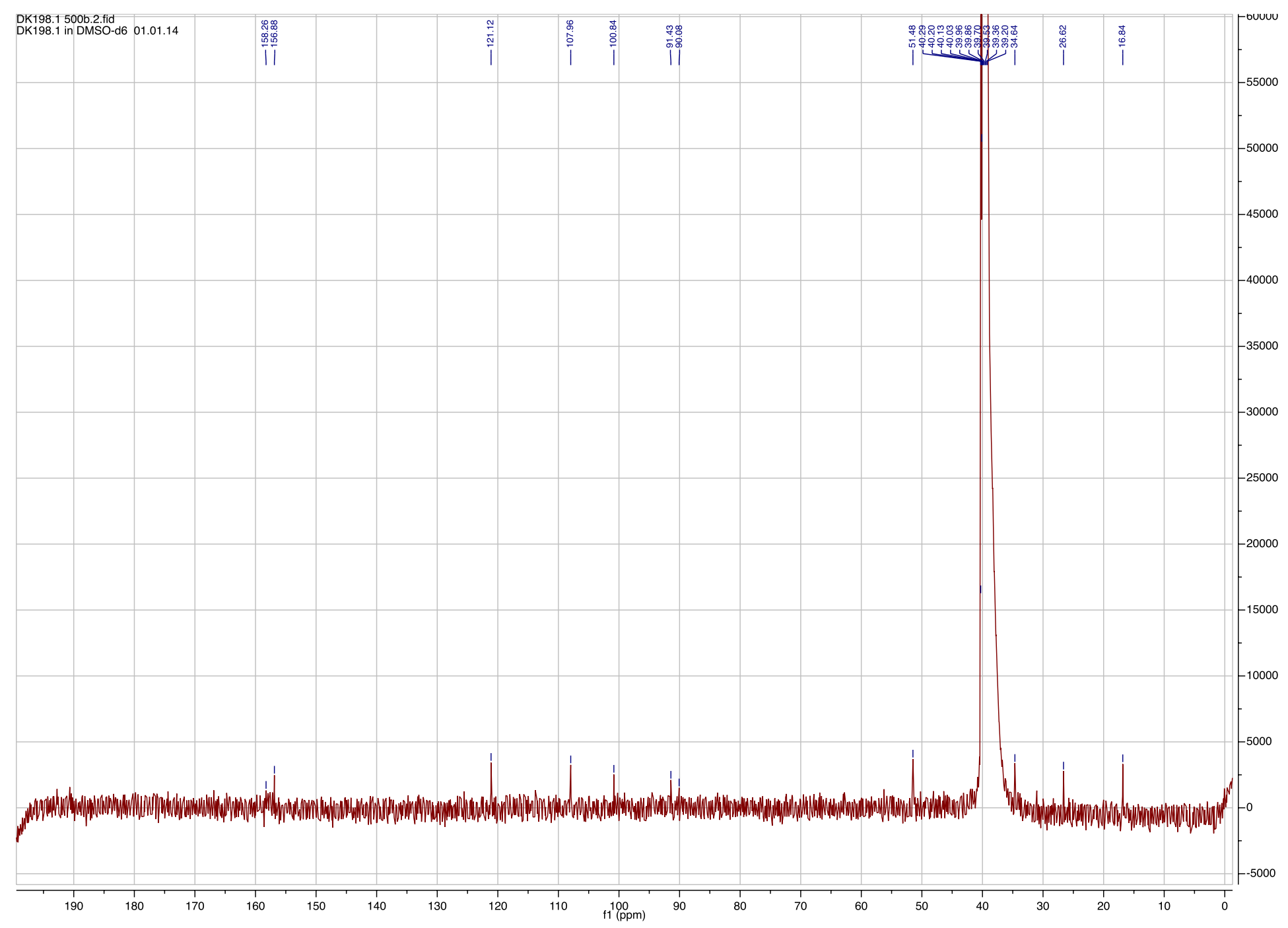


S46. HSQC Spectrum of (-)-11-O-Methylmonobromoagelaspongin (6) in DMSO- $d_{6}$

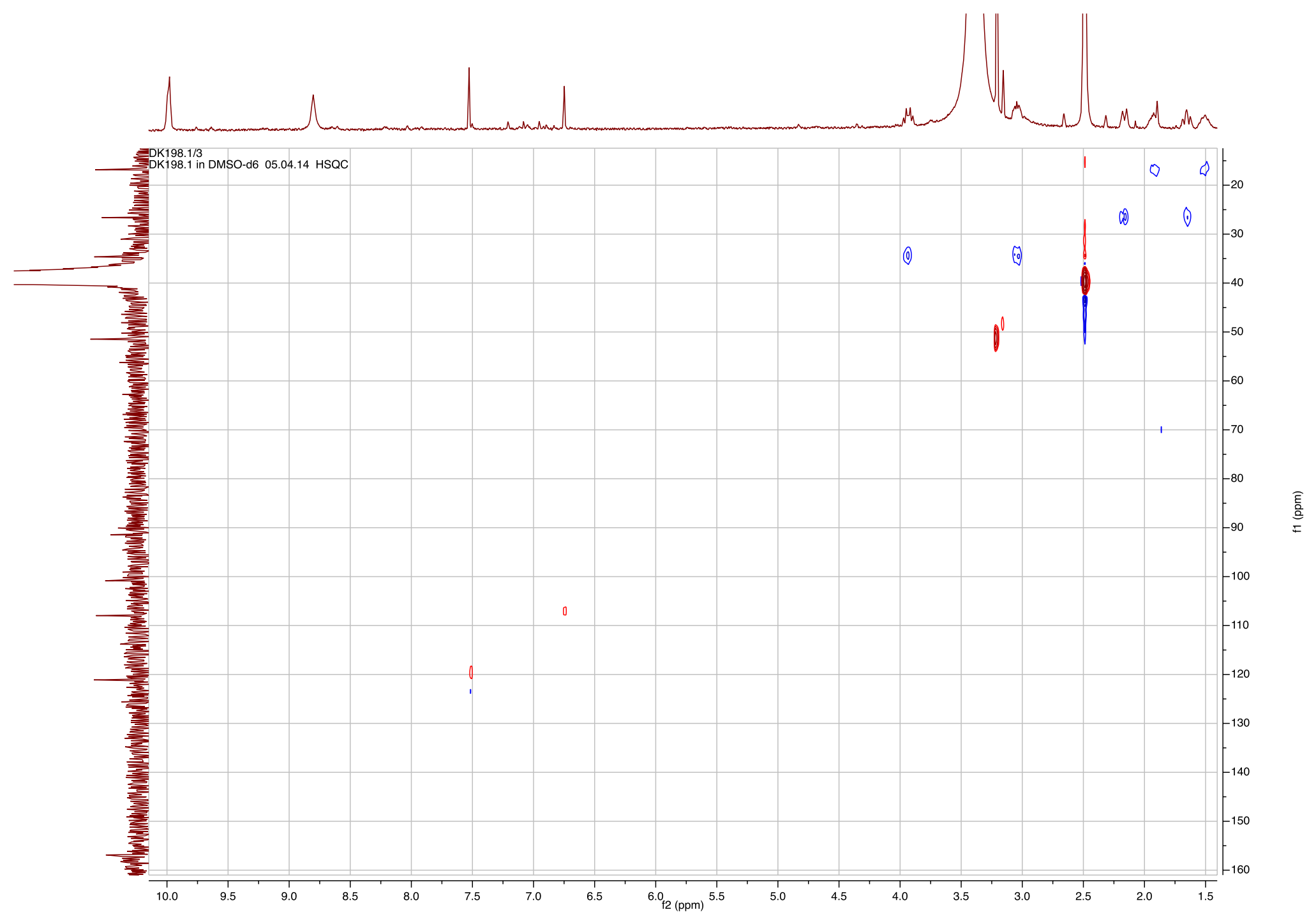


S47. HMBC Spectrum of (-)-11-O-Methylmonobromoagelaspongin (6) in DMSO- $d_{6}$

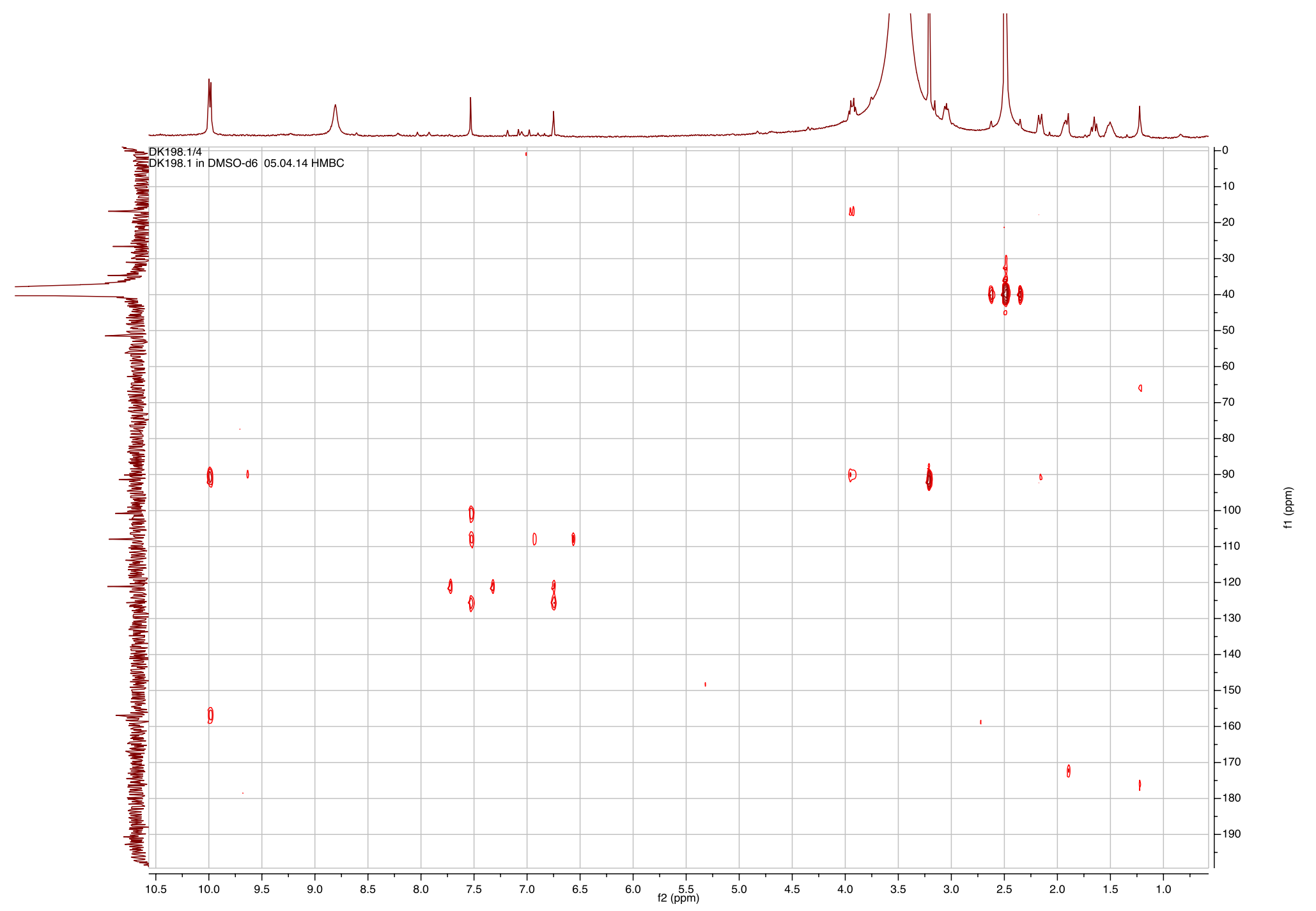


S48. COSY Spectrum of (-)-11-O-Methylmonobromoagelaspongin (6) in DMSO- $d_{6}$

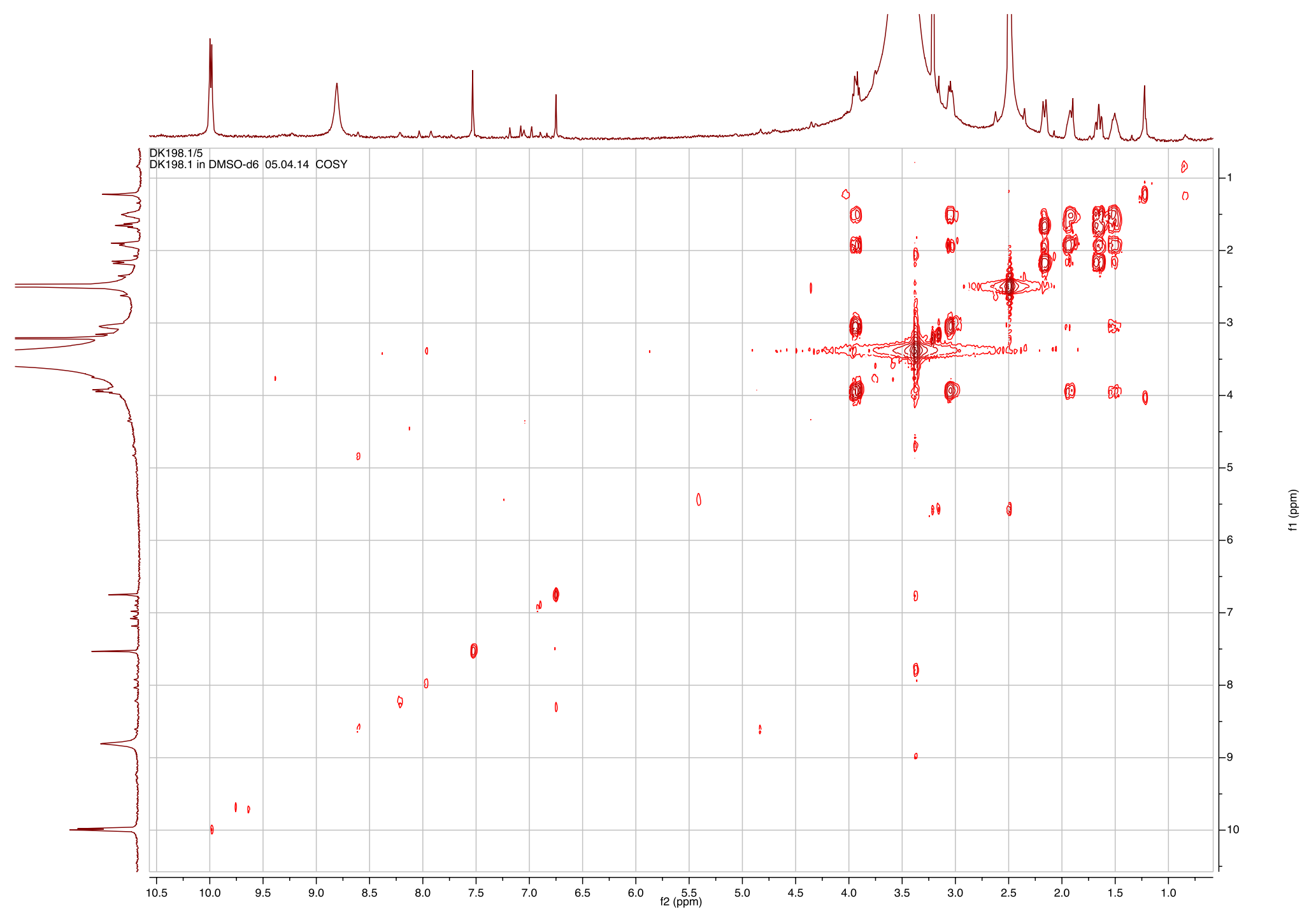


S49. Table S8. NMR Data (-)-11-O-Methylmonobromoagelaspongin (6) in DMSO- $d_{6}$

\begin{tabular}{|c|c|c|c|}
\hline Position & $\delta_{C}^{b}$ & $\delta_{\mathrm{H}}$, mult., $J(\mathrm{~Hz})$ & $\begin{array}{l}\text { HMBC } \\
\text { correlations }\end{array}$ \\
\hline 2 & $121.1, \mathrm{CH}$ & $7.53, \mathrm{~s}$ & 4 \\
\hline 3 & $100.9, \mathrm{C}$ & & 2 \\
\hline 4 & $108.0, \mathrm{CH}$ & $6.75, \mathrm{~s}$ & 2 \\
\hline 5 & $125.5, \mathrm{C}$ & & 2,4 \\
\hline 6 & $157.2, \mathrm{C}$ & & $8 \mathrm{a}$ \\
\hline $8 \mathrm{a}$ & $34.6, \mathrm{CH}_{2}$ & $3.93, \mathrm{dt}(12.5,8.5)$ & \\
\hline $\mathrm{b}$ & & $\begin{array}{l}\text { 3.04, ddd }(12.5,7.0 \text {, } \\
4.0)\end{array}$ & \\
\hline $9 \mathrm{a}$ & $16.8, \mathrm{CH}_{2}$ & $1.91, \mathrm{~m}$ & $8 \mathrm{a}, 10 \mathrm{a}$ \\
\hline $\mathrm{b}$ & & $1.50, \mathrm{~m}$ & \\
\hline 10a & 26.6, $\mathrm{CH}_{2}$ & $2.16, \mathrm{dt}(13.5,4.0)$ & \\
\hline $\mathrm{b}$ & & $1.64, \operatorname{td}(13.5,3.5)$ & \\
\hline 11 & $91.6, \mathrm{C}$ & & $11-\mathrm{OMe}, 12,14$ \\
\hline $\begin{array}{l}11- \\
\mathrm{OMe}\end{array}$ & $51.6, \mathrm{CH}_{3}$ & $3.16, \mathrm{~s}$ & \\
\hline 12 & & $9.99, \mathrm{~s}$ & \\
\hline 13 & 156.9, C & & 12,14 \\
\hline $13-\mathrm{NH}_{2}$ & & 8.80 , brs & \\
\hline 14 & & $9.98, \mathrm{~s}$ & \\
\hline 15 & $90.1, \mathrm{C}$ & & $8 \mathrm{a}, 12,14$ \\
\hline
\end{tabular}


S50. HR ESI MS data of (-)-11-O-Methylmonobromoagelaspongin (6)

Elemental Composition Report

Page 1

Single Mass Analysis

Tolerance $=5.0 \mathrm{mDa} /$ DBE: $\min =-1.5, \max =50.0$

iction: Off

作

Monoisotopic Mass, Even Electron lons

361 formula(e) evaluated with 8 results within limits (up to 4 best isotopic matches for each mass)

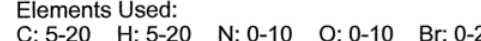

DK198.1 $13(0.596) \mathrm{Cm}(13: 14)$

Dimitry Kovalerchik

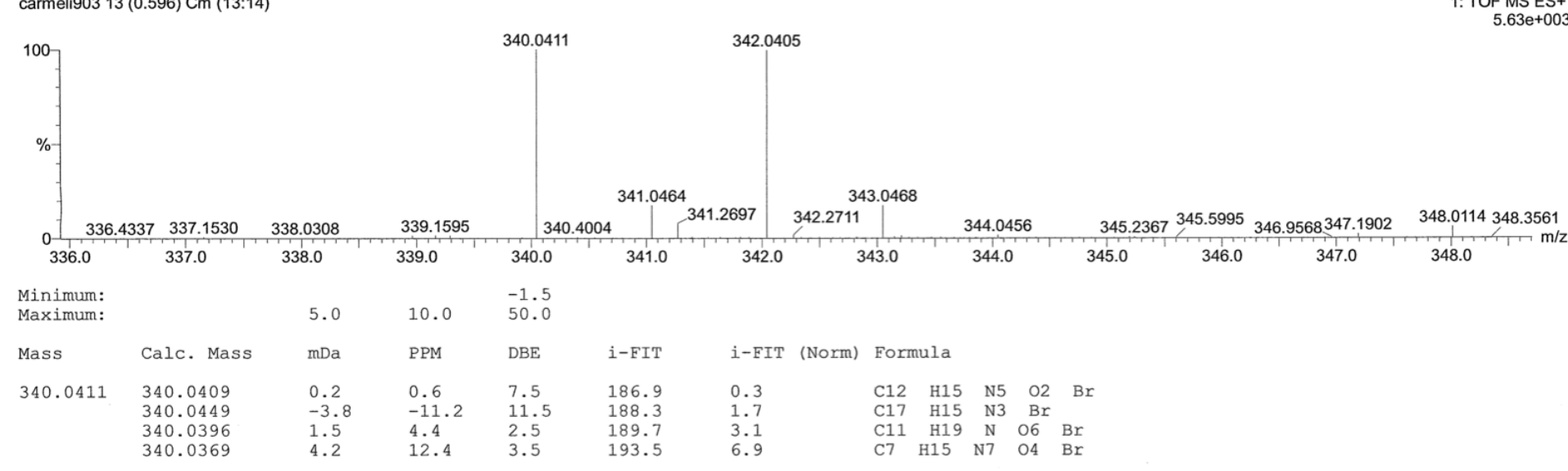

$5.63 \mathrm{e}+003$ 


$$
\frac{E n}{3}
$$


S52. ${ }^{13} \mathrm{C}$ NMR Spectrum of $E$-Dispacamide (7) in DMSO- $d_{6}$

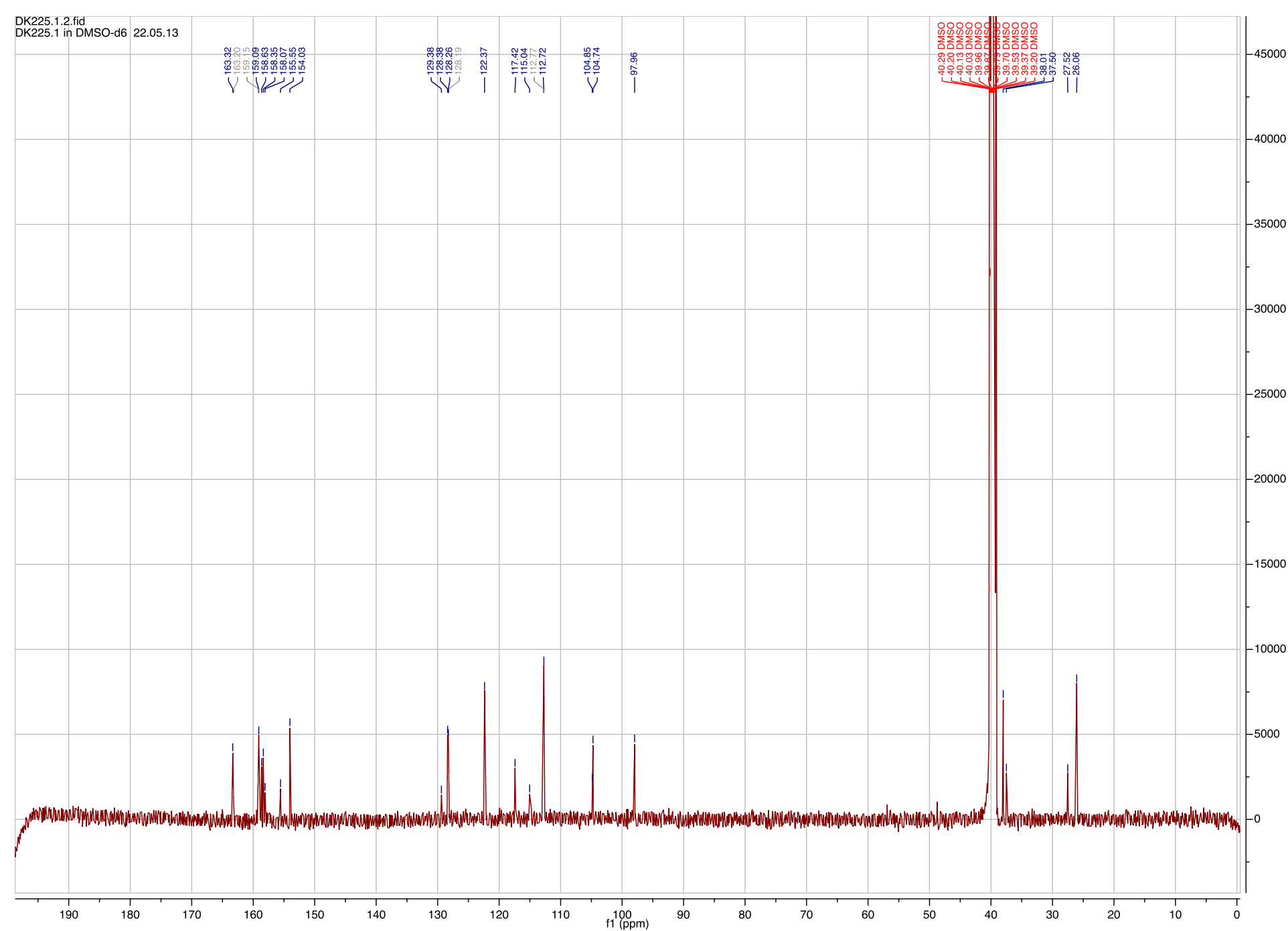


S53. HSQC Spectrum of $E$-Dispacamide (7) in DMSO- $d_{6}$

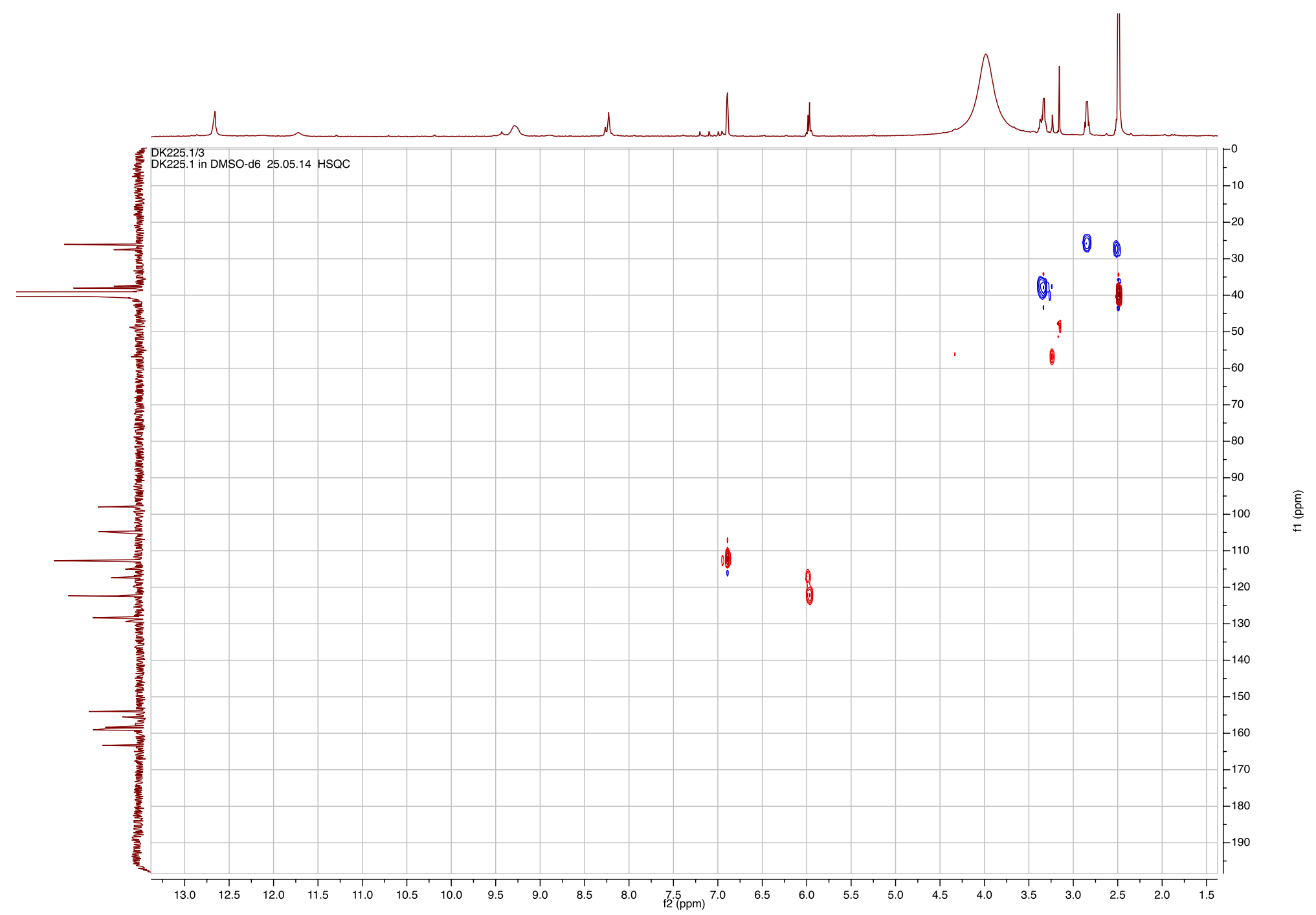


S54. HMBC Spectrum of $E$-Dispacamide (7) in DMSO- $d_{6}$

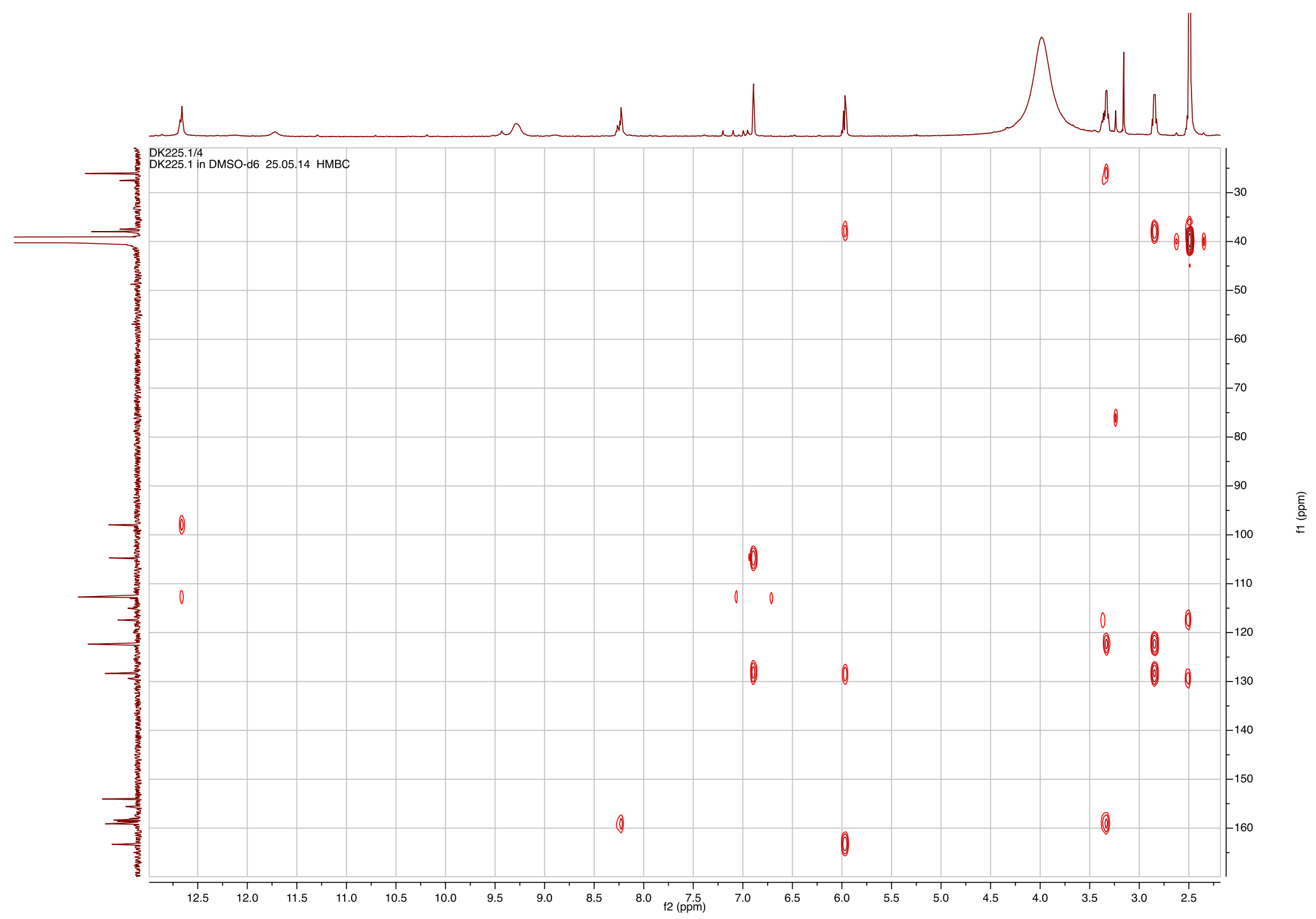


S55. COSY Spectrum of $E$-Dispacamide (7) in DMSO- $d_{6}$

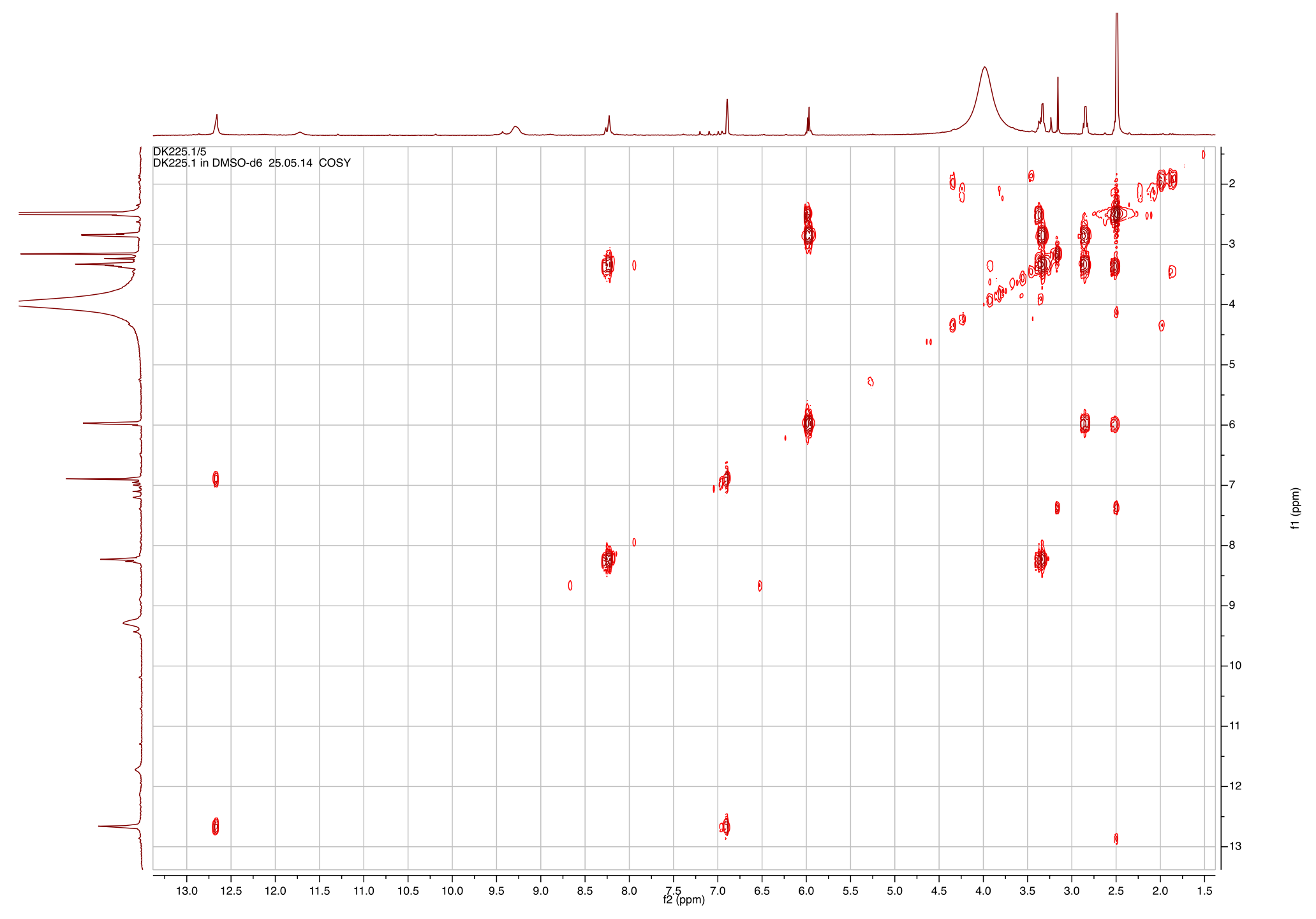


S56. ROESY Spectrum of $E$-Dispacamide (7) in DMSO- $d_{6}$

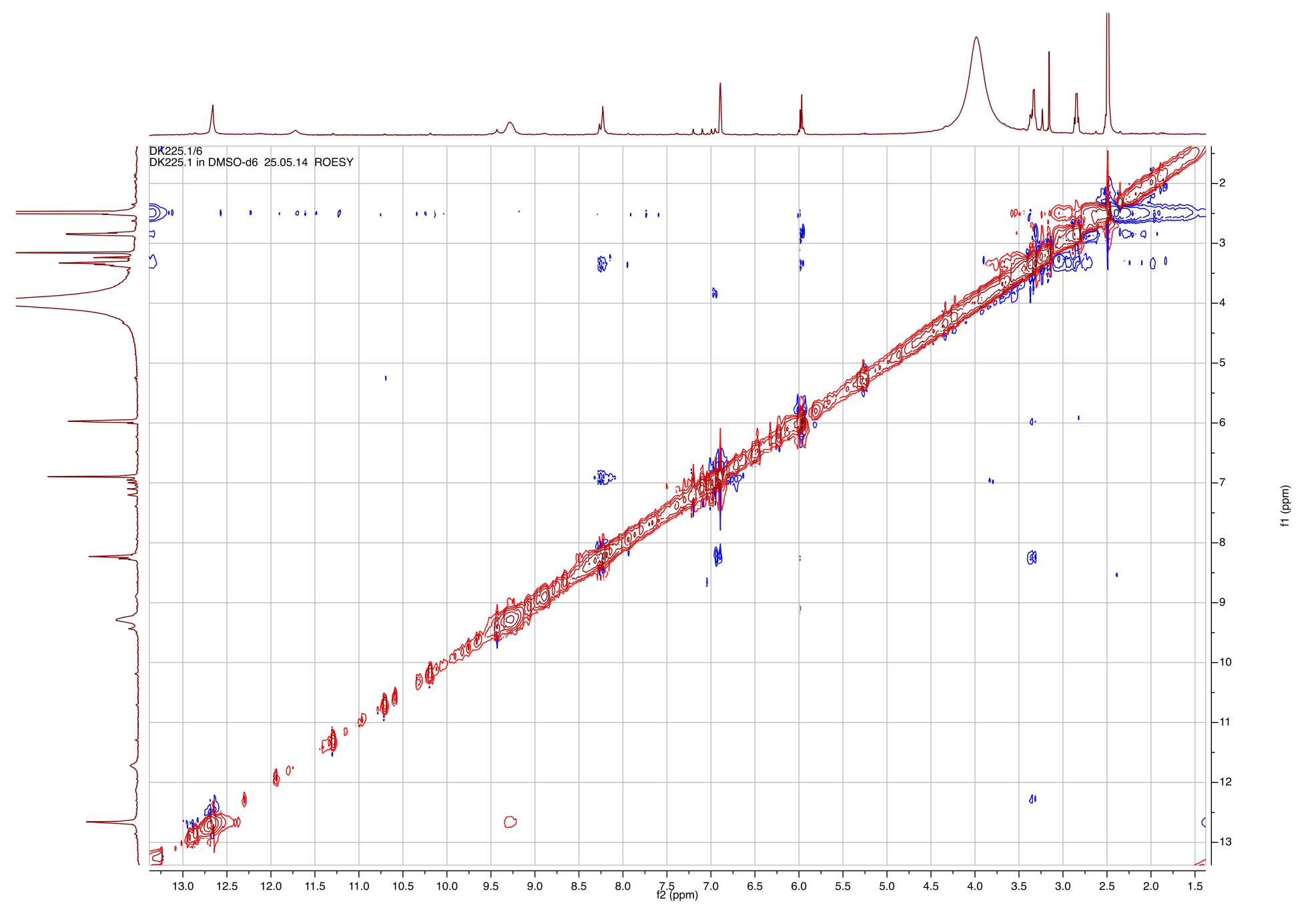


S57. ${ }^{1} \mathrm{H}$ NMR Spectrum of $E$-Dispacamide (7) in DMF- $d_{7}$ at $233{ }^{\circ} \mathrm{K}$

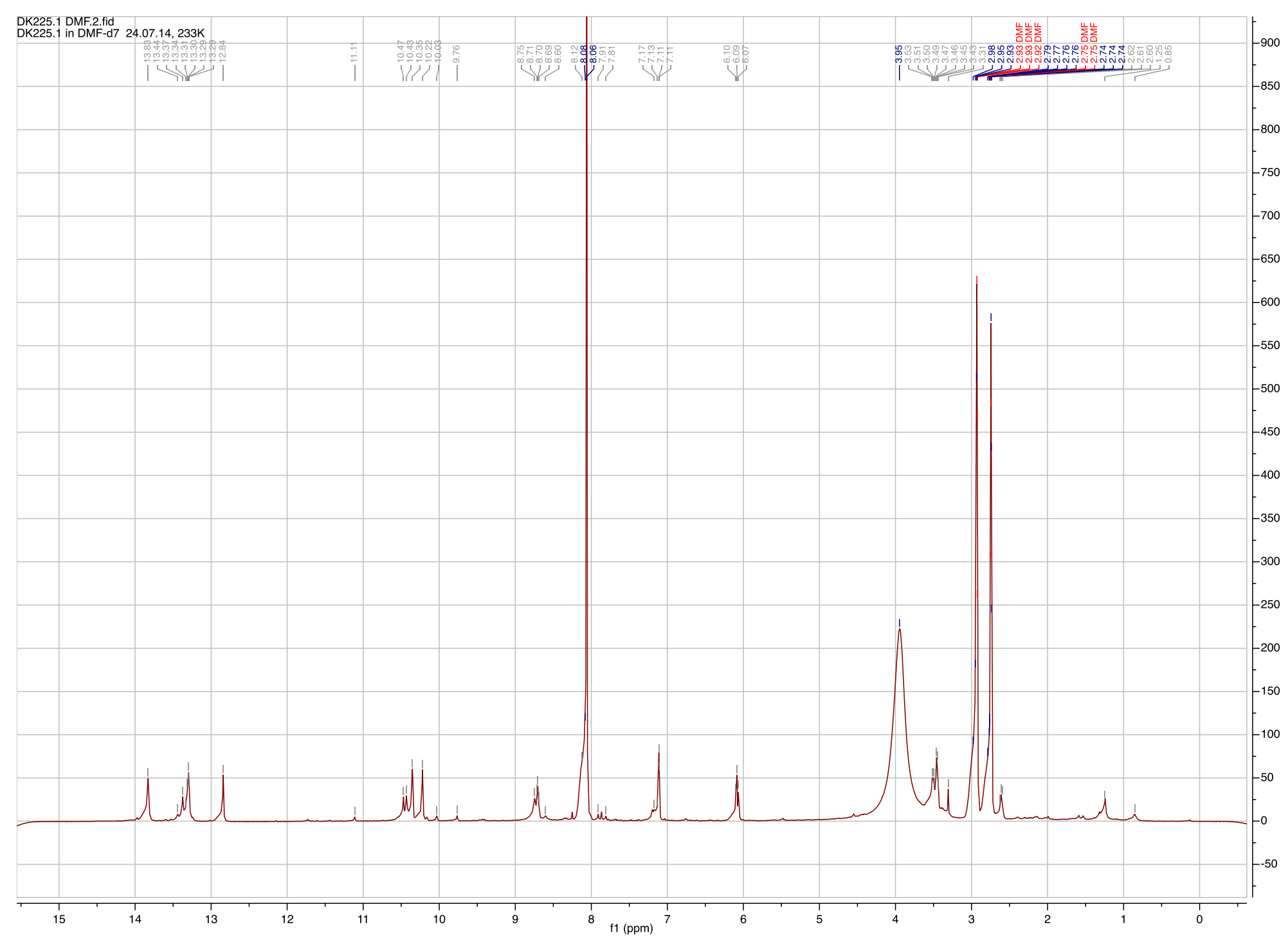


S58. Difference-NOE Spectra of $E$-Dispacamide (7) in DMF- $d_{7}$ at $233^{\circ} \mathrm{K}$

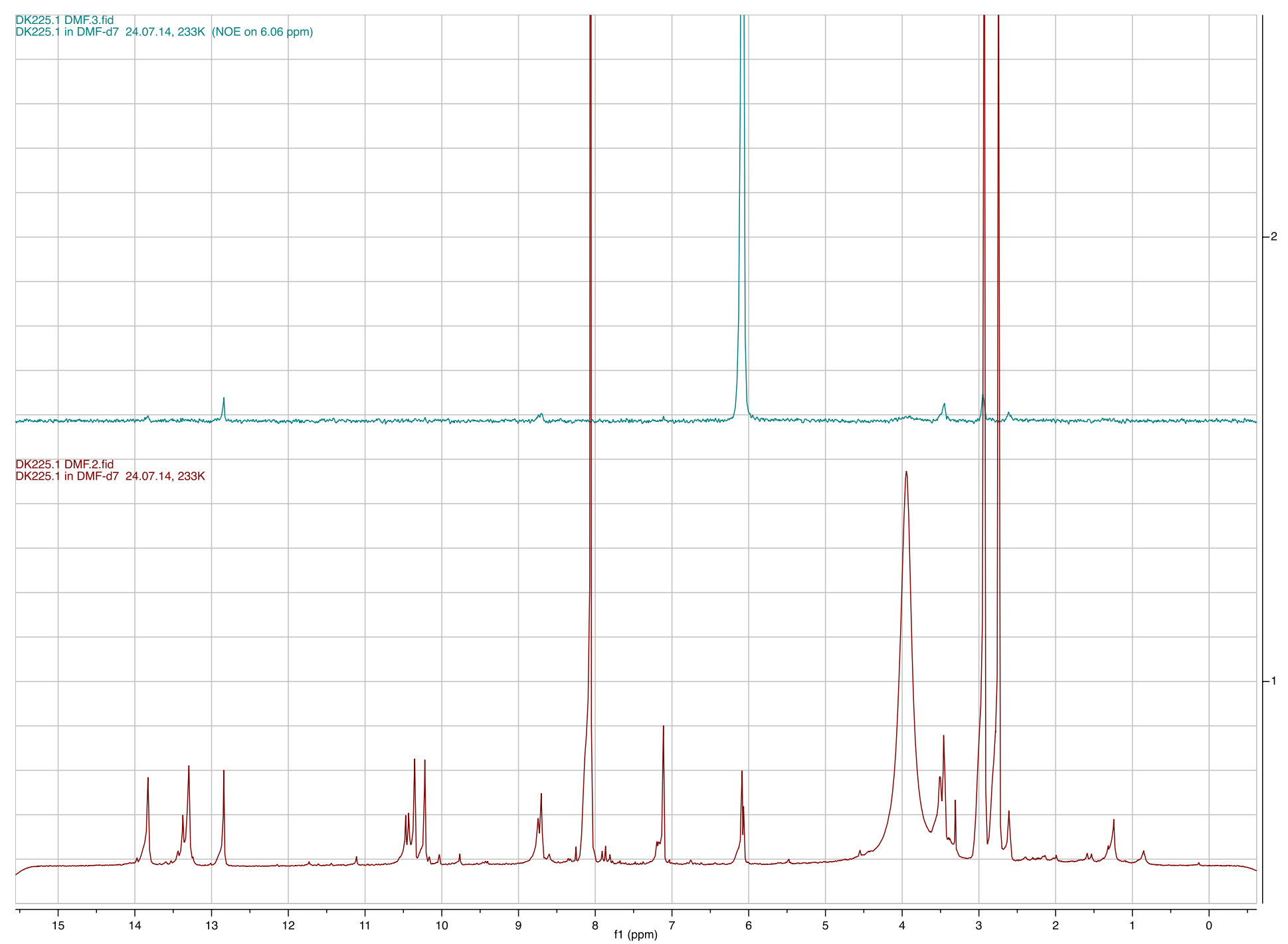


S59. Table S9. NMR Data of $E$-Dispacamide (7) in DMSO- $d_{6}$ and in DMF- $d_{7}$ at $233^{\circ} \mathrm{K}^{\mathrm{a}}$

\begin{tabular}{|c|c|c|c|c|c|}
\hline Position & $\delta_{\mathrm{C}^{\mathrm{b}}}$ & $\delta_{\mathrm{H}}$, mult., $J(\mathrm{~Hz})$ & $\delta_{\mathrm{H}}$, mult., $J(\mathrm{~Hz})^{\mathrm{c}}$ & HMBC correlations & NOE correlations \\
\hline 1 & & $12.65, \mathrm{~s}$ & $13.30, \mathrm{~s}$ & & \\
\hline 2 & 104.7, C & & & 4 & \\
\hline 3 & $98.0, \mathrm{C}$ & & & 1 & \\
\hline 4 & $112.7, \mathrm{CH}$ & $6.89, \mathrm{~d}(2.6)$ & $7.11, \mathrm{~s}$ & 1 & 7 \\
\hline 5 & 128.1, C & & & 1,4 & \\
\hline 6 & $159.1, \mathrm{C}$ & & & 7 & \\
\hline 7 & & $8.22, \mathrm{t}(6.5)$ & 8.72 , brs & & $4,8,9$ \\
\hline 8 & $38.0, \mathrm{CH}_{2}$ & $3.33, \mathrm{q}(6.5)$ & $3.47, \mathrm{q}(7.0)$ & 7,9 & 7,9 \\
\hline 9 & $26.1, \mathrm{CH}_{2}$ & $2.85, \mathrm{q}(6.5)$ & $2.95, \mathrm{q}(7.0)$ & 8 & 8 \\
\hline 10 & $122.4, \mathrm{CH}$ & $5.97, \mathrm{t}(6.5)$ & $6.08, \mathrm{t}(7.0)$ & 8,9 & $8,9,12^{\mathrm{d}}$ \\
\hline 11 & $128.2, \mathrm{C}$ & & & 9,10 & \\
\hline 12 & & 9.28, brs & $12.84, \mathrm{~s}$ & & $10^{\mathrm{d}}$ \\
\hline 13 & 154.0, C & & & & \\
\hline $13-\mathrm{NH}_{2}$ & & 9.28, brs & $10.43, \mathrm{~s}, 10.46, \mathrm{~s}$ & & \\
\hline 14 & & $9.28, \mathrm{brs}$ & $13.83, \mathrm{~s}$ & & \\
\hline 15 & 163.3, C & & & 10 & \\
\hline
\end{tabular}

a 500.13 MHz for ${ }^{1} \mathrm{H}$ and $125.76 \mathrm{MHz}$ for ${ }^{13} \mathrm{C}$. ${ }^{b}$ Multiplicity and assignment from HSQC experiment.

${ }^{\mathrm{c}}$ Measured in DMF- $d_{7}$ at $233^{\circ} \mathrm{K}$. ${ }^{\mathrm{d}} \mathrm{NOE}$ 's measured in DMF- $d_{7}$ at $233^{\circ} \mathrm{K}$. 
S60. Table S10. Comparison of the NMR Data (500 MHz, DMSO- $d_{6}$ ) for $E$-Dispacamide (7) and Dispacamide

\begin{tabular}{|c|c|c|c|c|}
\hline position & $E$-dispacan & & dispacamid & \\
\hline & $\delta_{\mathrm{C}}$, type & $\delta_{\mathrm{H}}(J$ in $\mathrm{Hz})$ & $\delta_{\mathrm{C}}$, type & $\delta_{\mathrm{H}}(J$ in $\mathrm{Hz})$ \\
\hline 1 & & $12.65, \mathrm{~s}$ & & $12.69, \mathrm{~s}$ \\
\hline 2 & 104.7, C & & 104.9, C & \\
\hline 3 & 98.0, C & & 98.0, C & \\
\hline 4 & $112.7, \mathrm{CH}$ & $6.89, \mathrm{~d}(2.6)$ & $112.8, \mathrm{CH}$ & $6.89, \mathrm{~d}(2.0)$ \\
\hline 5 & 128.1, C & & $128.2, \mathrm{C}$ & \\
\hline 6 & 159.1, C & & $159.2, \mathrm{C}$ & \\
\hline 7 & & $8.22, \mathrm{t}(6.5)$ & & $8.27, \mathrm{t}(5.5)$ \\
\hline 8 & $38.0, \mathrm{CH}_{2}$ & $3.33, q(6.5)$ & $37.5, \mathrm{CH}_{2}$ & $3.37, \mathrm{q}(6.0)$ \\
\hline 9 & 26.1, $\mathrm{CH}_{2}$ & $2.85, q(6.5)$ & $27.5, \mathrm{CH}_{2}$ & $2.52, q(6.5)$ \\
\hline 10 & 122.4, $\mathrm{CH}$ & $5.97, \mathrm{t}(6.5)$ & 117.3, СН & $5.98, t(7.5)$ \\
\hline 11 & 128.2, C & & $129.5, \mathrm{C}$ & \\
\hline 12 & & $9.28, \mathrm{brs}$ & & $9.29 \mathrm{brs}$ \\
\hline 13 & $154.0, \mathrm{C}$ & & 155.7, C & \\
\hline $13-\mathrm{NH}_{2}$ & & 9.28 , brs & & $9.29 \mathrm{brs}$ \\
\hline 14 & & 9.28 , brs & & $9.29 \mathrm{brs}$ \\
\hline 15 & $163.3, \mathrm{C}$ & & $163.4, \mathrm{C}$ & \\
\hline
\end{tabular}


S61. HR ESI MS data of $E$-Dispacamide (7) in DMSO- $d_{6}$

\section{Elemental Composition Report}

Page 1

Single Mass Analysis

Tolerance $=5.0 \mathrm{mDa} / \mathrm{DBE}: \min =-1.5, \max =50$.

Number of isotope peaks used for $\mathrm{i}-\mathrm{FIT}=3$

Monoisotopic Mass, Even Electron lons

268 formula(e) evaluated with 9 results within limits (up to 4 closest results for each mass)

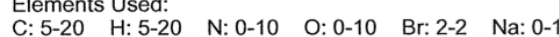

DK225.1

carmeli920 84 (3.699) $\mathrm{Cm}$ (75:88)

Dimitry Kovalerchik

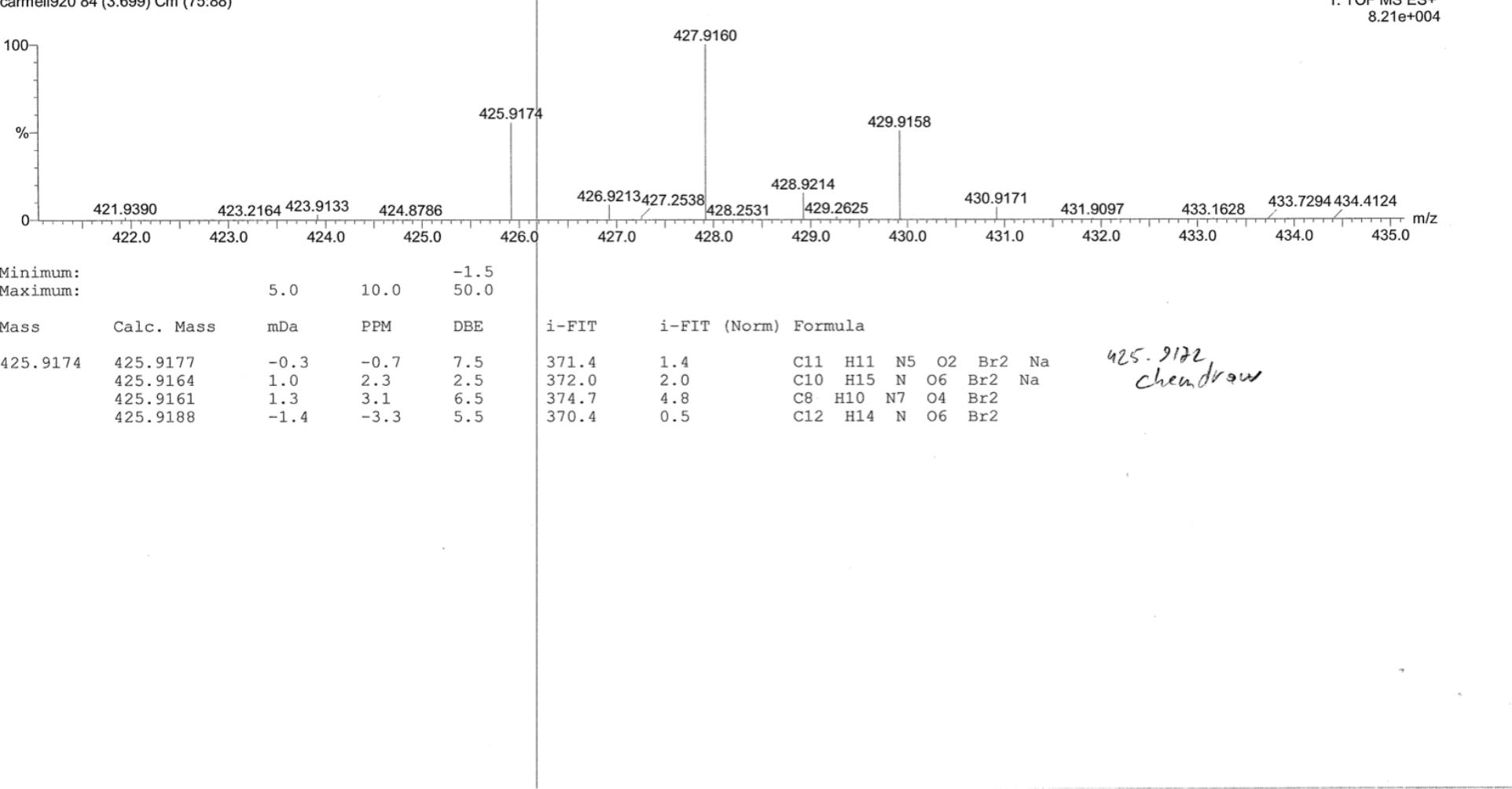


S62. ${ }^{1} \mathrm{H}$ NMR Spectrum of Pyrrolosine (8) in DMSO- $d_{6}$

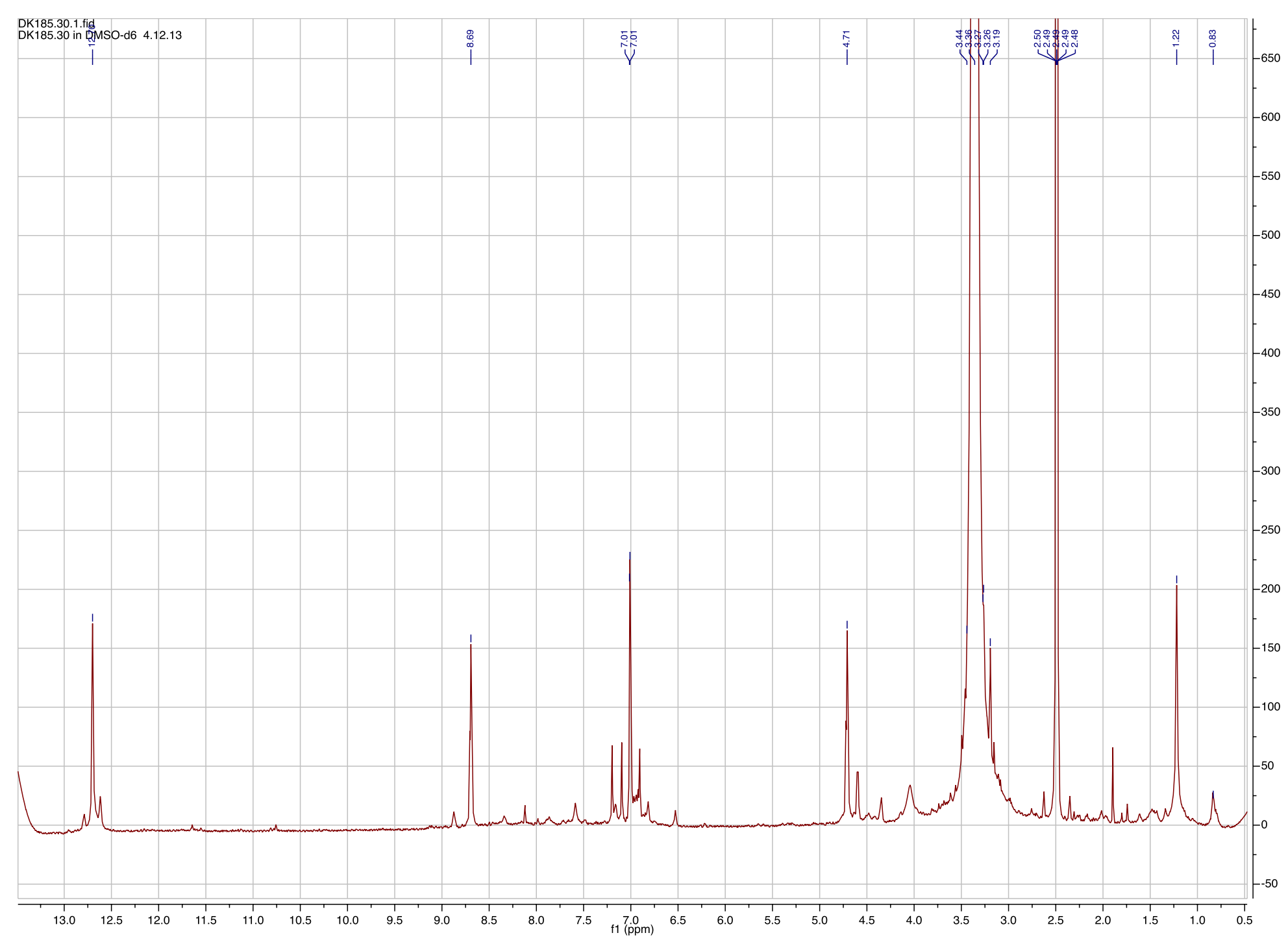


S63. ${ }^{13}$ C NMR Spectrum of Pyrrolosine (8) in DMSO- $d_{6}$

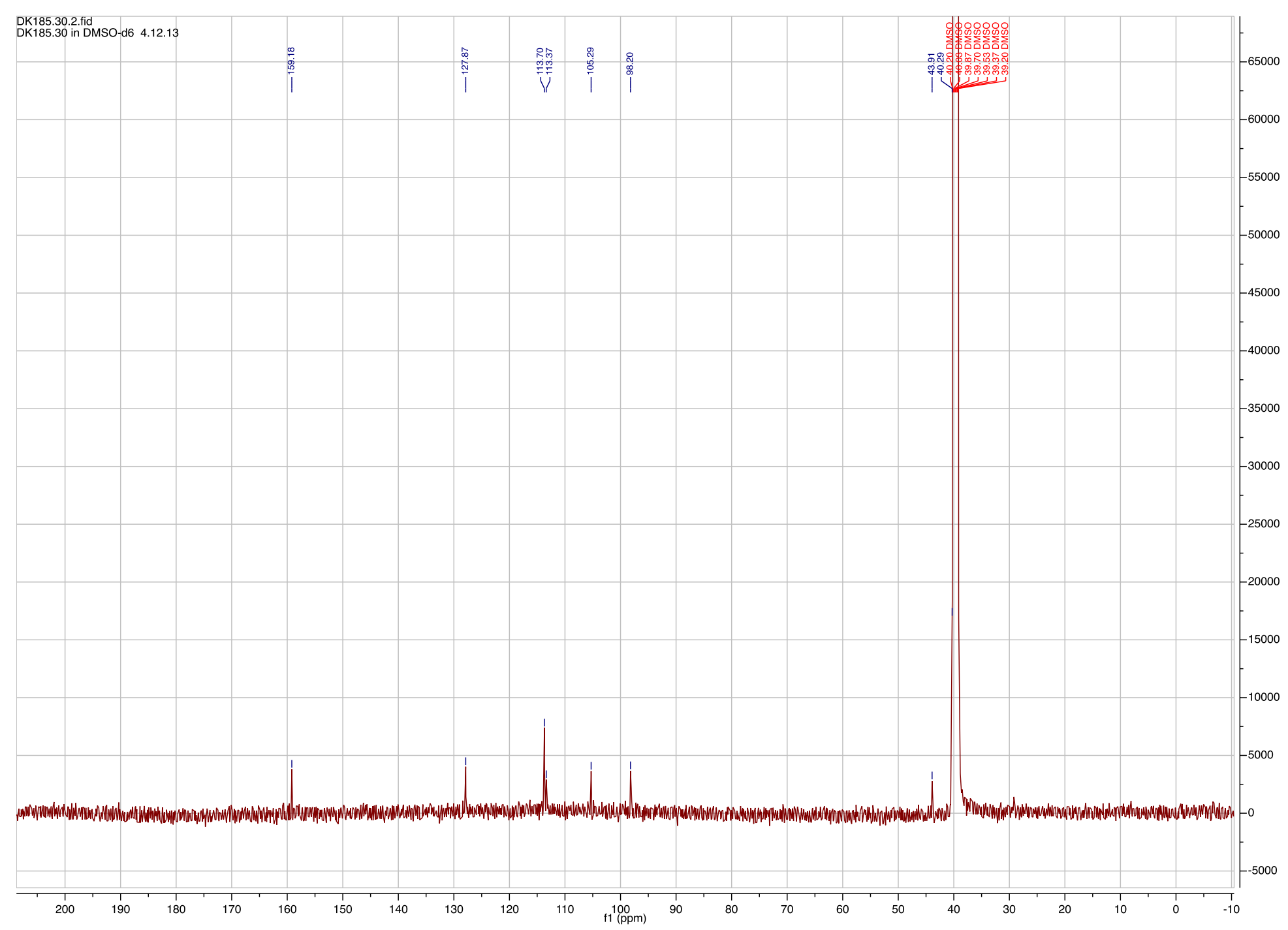


S64. HSQC Spectrum of Pyrrolosine (8) in DMSO- $d_{6}$

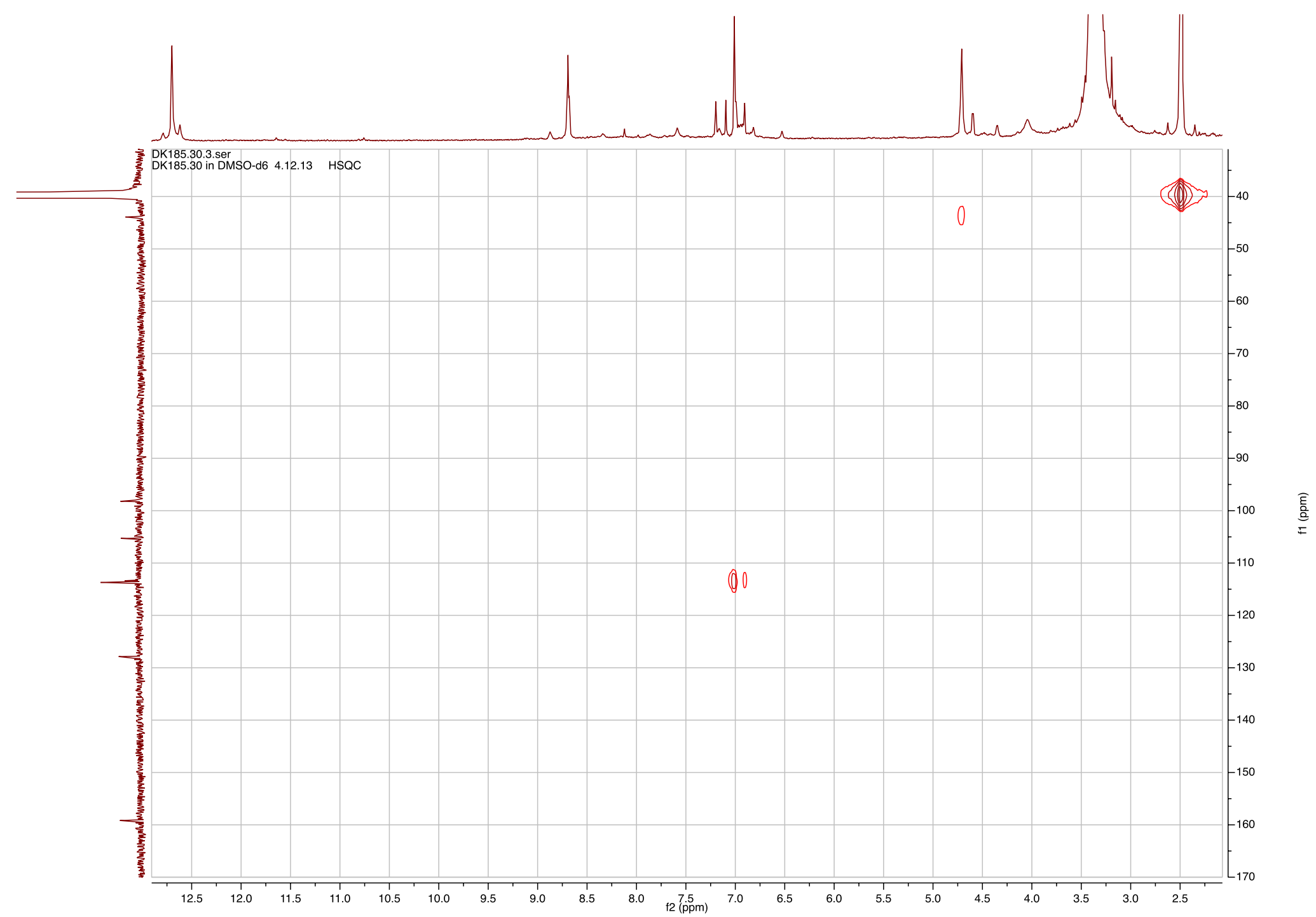


S65. HMBC Spectrum of Pyrrolosine (8) in DMSO- $d_{6}$

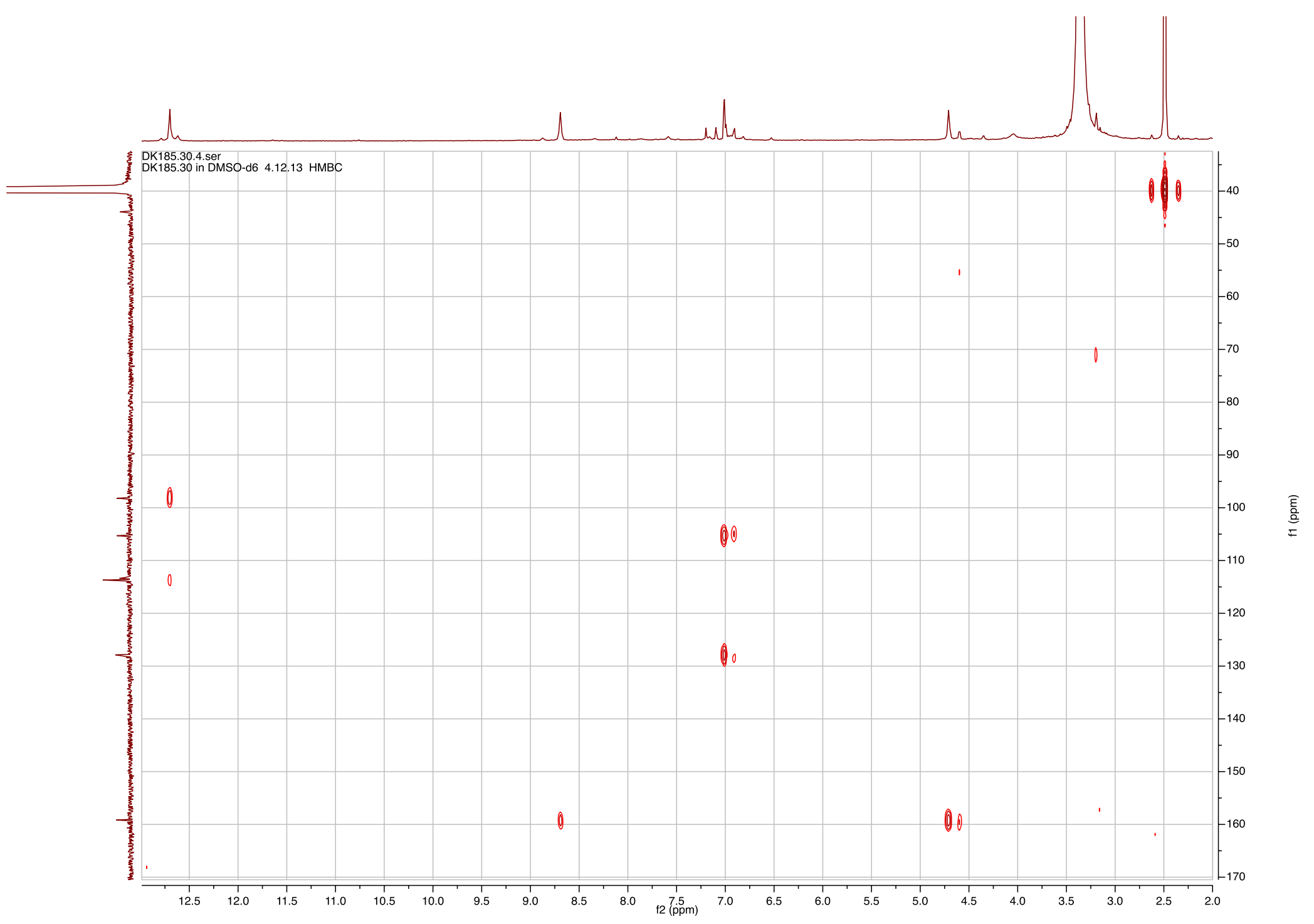


S66. COSY Spectrum of Pyrrolosine (8) in DMSO- $d_{6}$

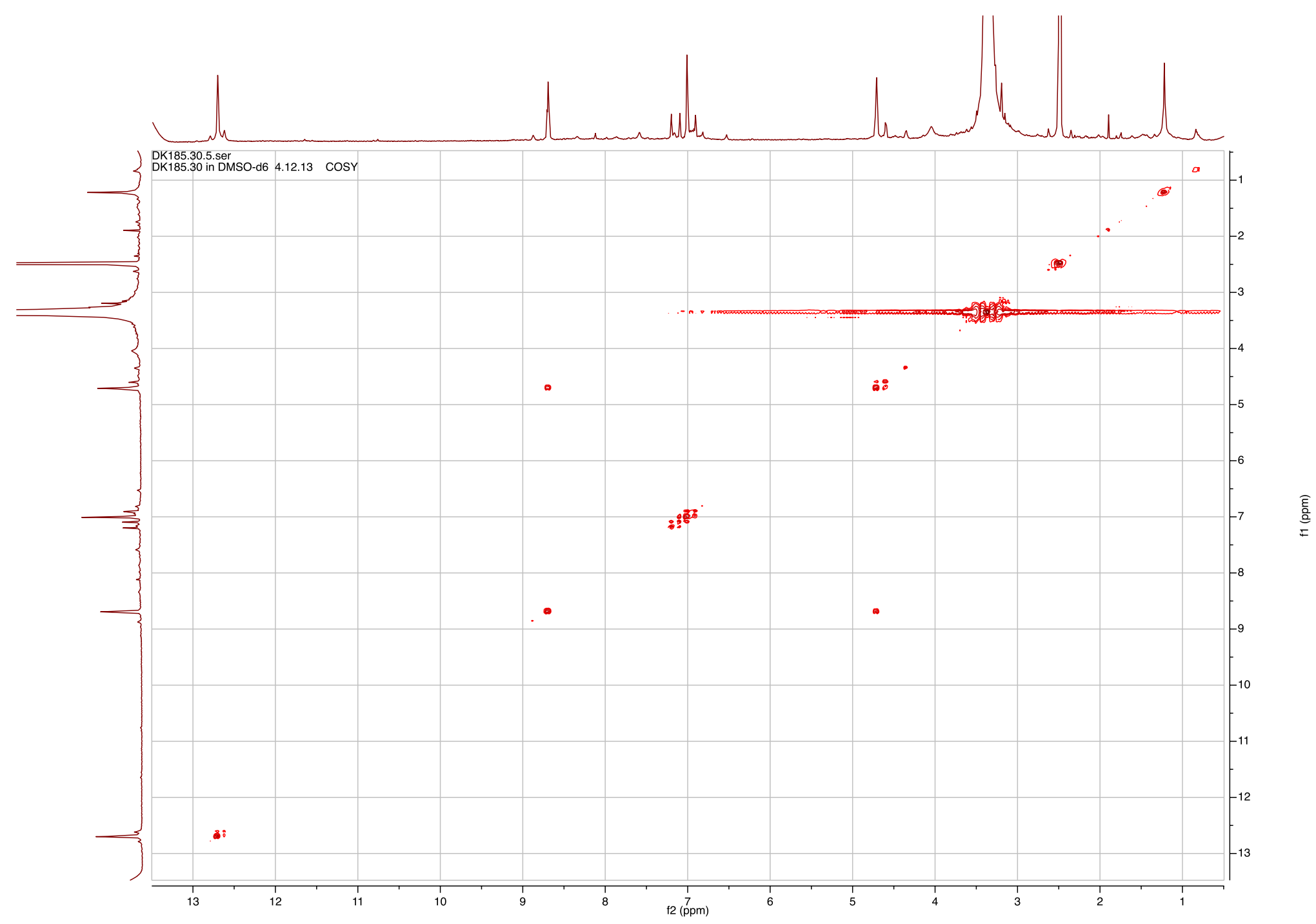


S67. Table S11. NMR Data of Pyrrolosine (8) in DMSO- $d_{6}{ }^{\mathrm{a}}$

\begin{tabular}{llll}
\hline Position & $\delta_{\mathrm{C}} \mathrm{b}$ & $\delta_{\mathrm{H}}$, mult., $J(\mathrm{~Hz})$ & HMBC correlations \\
\hline 1 & & $12.70, \mathrm{~s}$ \\
2 & $105.3, \mathrm{qC} \times 2$ & 4 \\
3 & $98.2, \mathrm{qC} \times 22$ & 1 \\
4 & $113.7, \mathrm{CH} \times 2$ & $7.00, \mathrm{~d}, 2.0$ & 1 \\
5 & $127.9, \mathrm{qC} \times 2$ & 4 \\
6 & $159.2, \mathrm{qC} \times 2$ & 7,8 \\
7 & & $8.69, \mathrm{t}, 5.5$ & \\
8 & $43.9, \mathrm{CH}_{2}$ & $4.71, \mathrm{t}, 5.5$ & \\
\hline
\end{tabular}

${ }^{\mathrm{a}} 500.13 \mathrm{MHz}$ for ${ }^{1} \mathrm{H}$ and $125.76 \mathrm{MHz}$ for ${ }^{13} \mathrm{C}$. ${ }^{\mathrm{b}}$ Multiplicity and assignment from HSQC experiment. 
S68. HR ESI MS data of Pyrrolosine (8)

Elemental Composition Report

Page 1

Single Mass Analysis
Tolerance $=5.0 \mathrm{mDa} / \mathrm{DBE}: \min =-1.5, \max =50.0$

Number of isotope peaks used for i-FIT $=3$

Monoisotopic Mass, Even Electron Ions

1193 formula(e) evaluated with 6 results within limits (up to 4 closest results for each mass)

$\begin{array}{lllll}\text { Elements Used: } & \text { N: } \\ \text { C: } 5-15 & \text { H: } 5-20 & \text { O: 0-10 Na: 0-1 } & \text { Br: 0-4 }\end{array}$

DK185.30

Dimitry Kovalerchik

carmeli907 138 (6.066) Cm (137:138)

1: TOF MS ES+
$8.26 \mathrm{e}+002$

100

570.7228

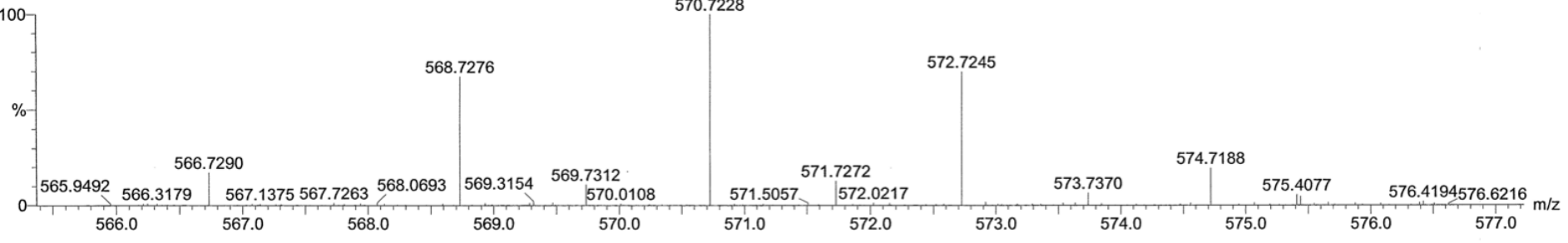

$5057, \quad 572.0217$

$573.0 \quad 574.0$

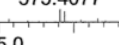

Minimum:

$\begin{array}{lll}5.0 & 10.0 & -1.5 \\ & 50.0\end{array}$

Mass Calc. Mass $\mathrm{mDa}$ PPM

$\begin{array}{llllllllllll}566.7290 & 566.7289 & 0.1 & 0.2 & 5.5 & 59.0 & 1.2 & \text { C12 } & \text { H11 } & \text { O6 } & \text { Br4 } \\ & 566.7302 & -1.2 & -2.1 & 10.5 & 60.2 & 2.3 & \text { C13 } & \text { H7 } & \text { N4 } & \text { O2 } & \text { B } x 4\end{array}$

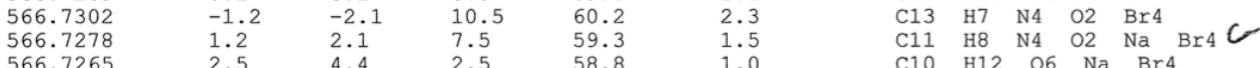


S69. Physiochemical data of Agesamide A

Agesamide A: Amorphous white solid; $[\alpha]^{25} \mathrm{D}-6.2$ (c 0.58 DMSO); UV (MeOH) $\lambda_{\max }(\log \varepsilon) 204 \mathrm{~nm}(4.26), 235 \mathrm{~nm}(3.96), 283 \mathrm{~nm}(3.97)$; ECD (c $0.124 \mathrm{mM}, \mathrm{MeCN}) \lambda_{\max }(\Delta \varepsilon) 262$ (0.80), 235 (-0.57), 228 (-0.55) and 214 (-0.03) cm²/mol; IR (ATR Diamond) $v_{\max } 3177,3041,2360$, 2342, 1767, 1709, $1649 \mathrm{~cm}^{-1} ;{ }^{1} \mathrm{H}$ and ${ }^{13} \mathrm{C}$ NMR, Table S12; HRESIMS $m / z 426.9015$ [M+Na] ${ }^{+}$(calcd for $\mathrm{C}_{11} \mathrm{H}_{10} \mathrm{Br}_{2} \mathrm{~N}_{4} \mathrm{NaO}_{3}, 426.9017$ ). 
S70. ${ }^{1} \mathrm{H}$ NMR Spectrum of Agesamide A in DMSO- $d_{6}$

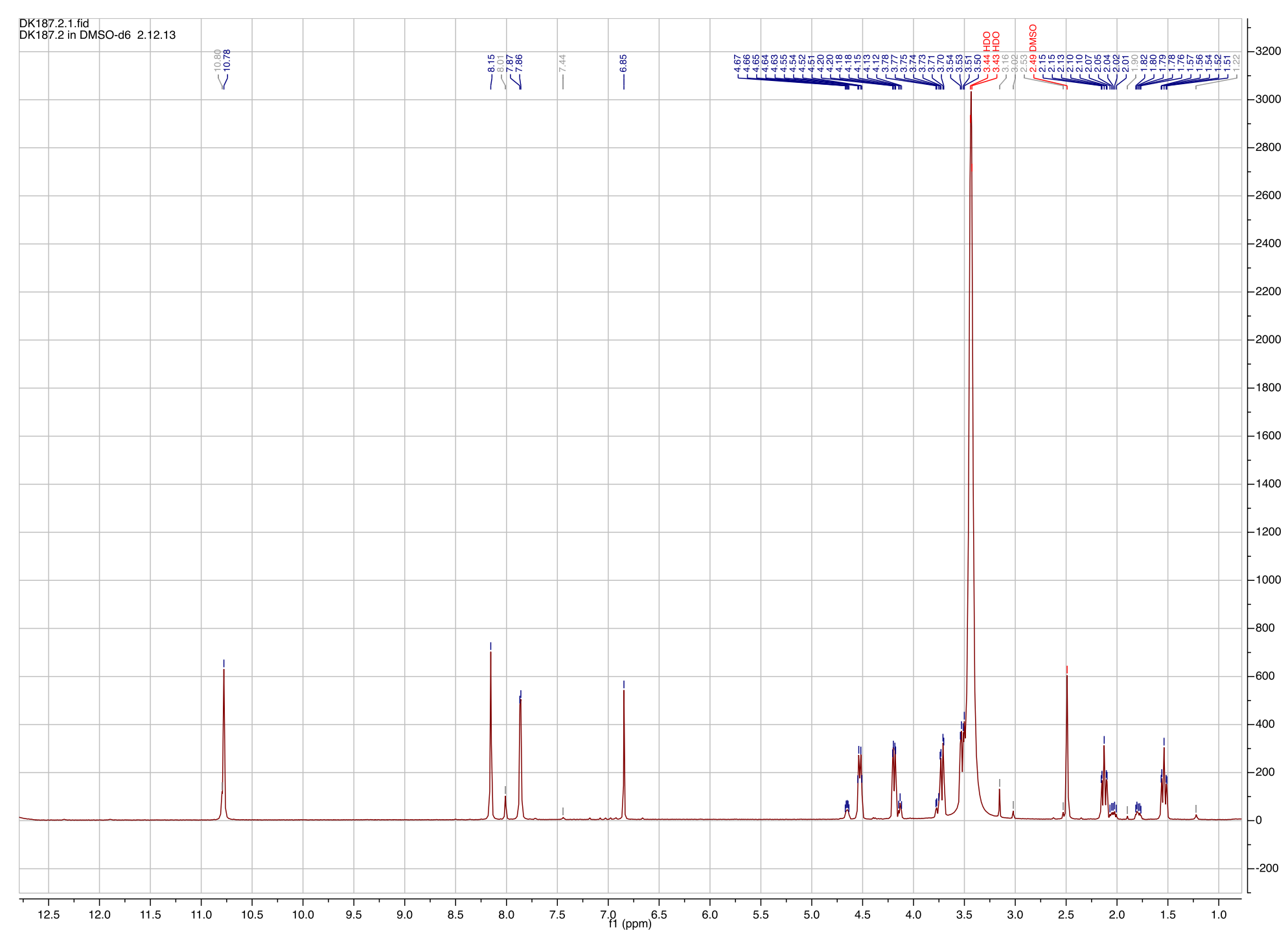


S71. ${ }^{13}$ C NMR Spectrum of Agesamide A in DMSO- $d_{6}$

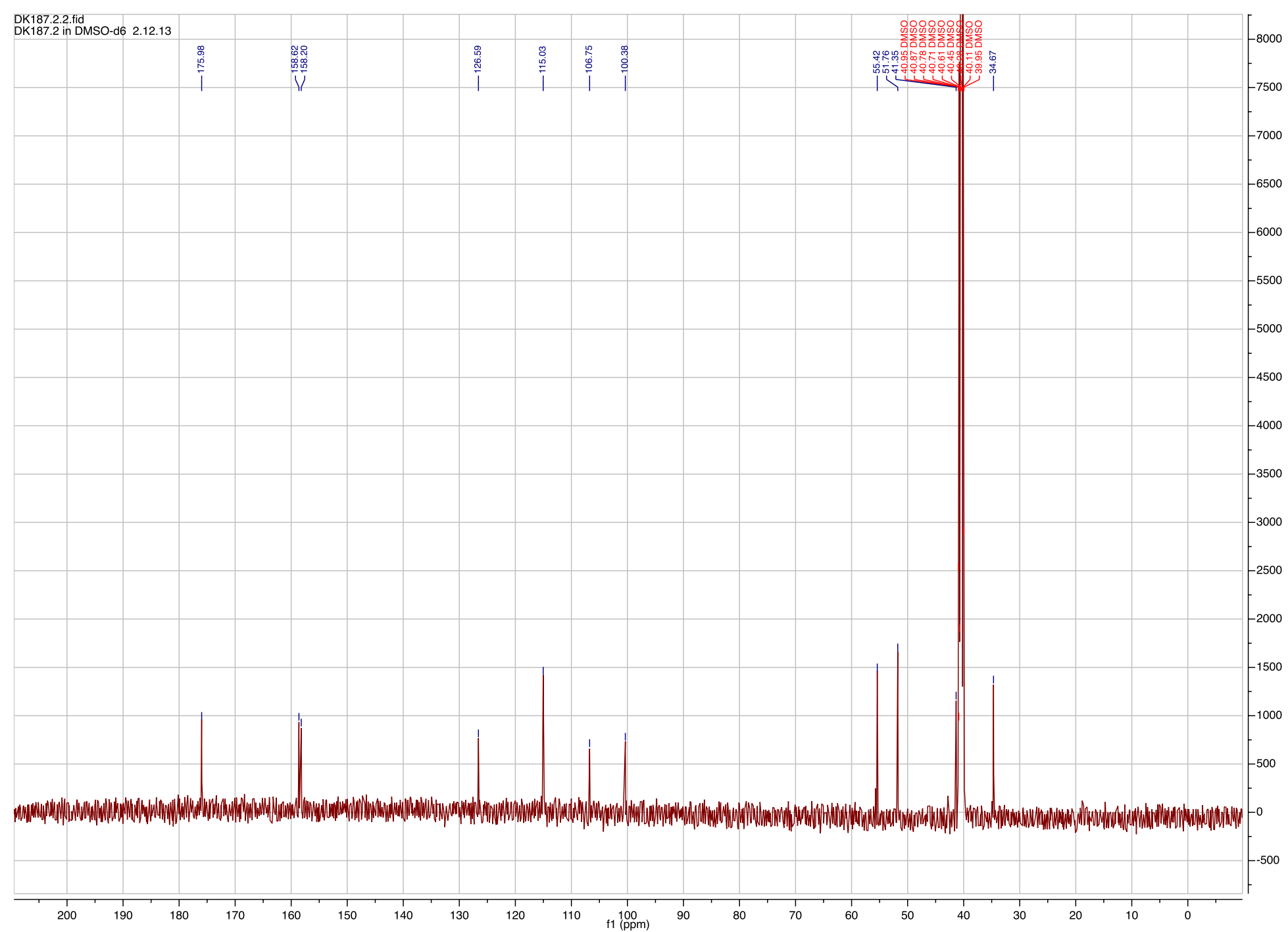


S72. HSQC Spectrum of Agesamide A in DMSO- $d_{6}$

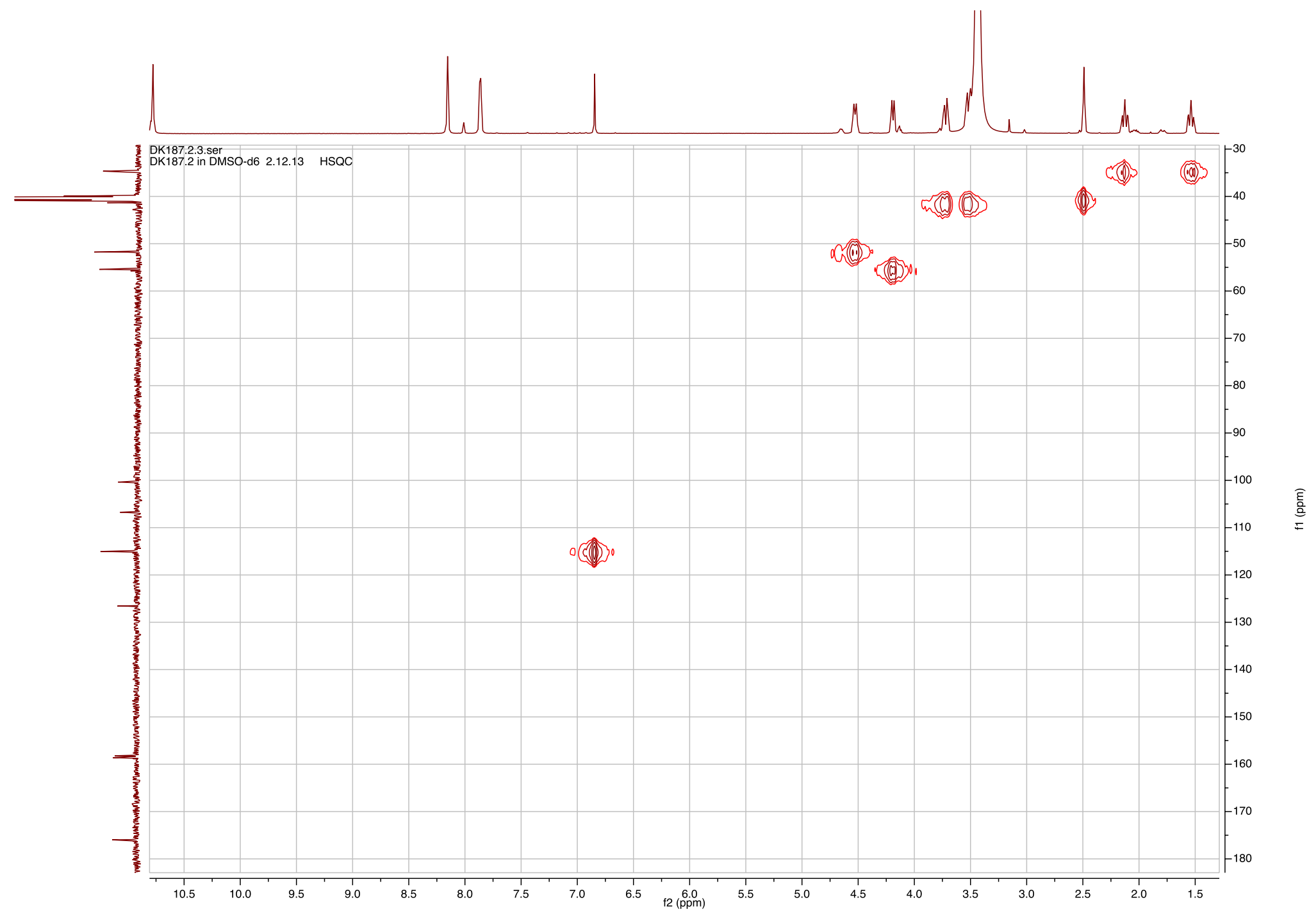


S73. HMBC Spectrum of Agesamide A in DMSO- $d_{6}$

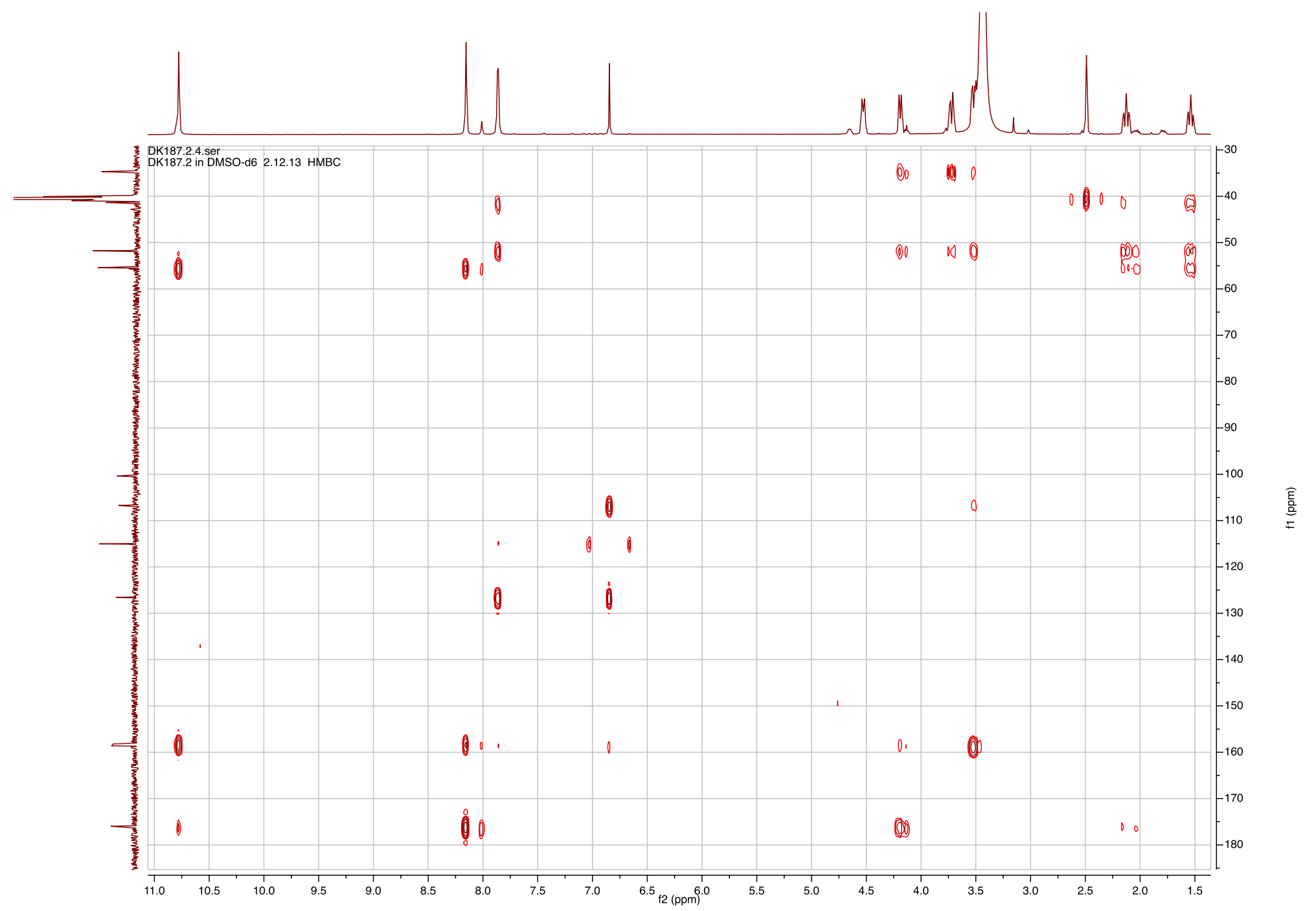


S74. COSY Spectrum of Agesamide A in DMSO- $d_{6}$

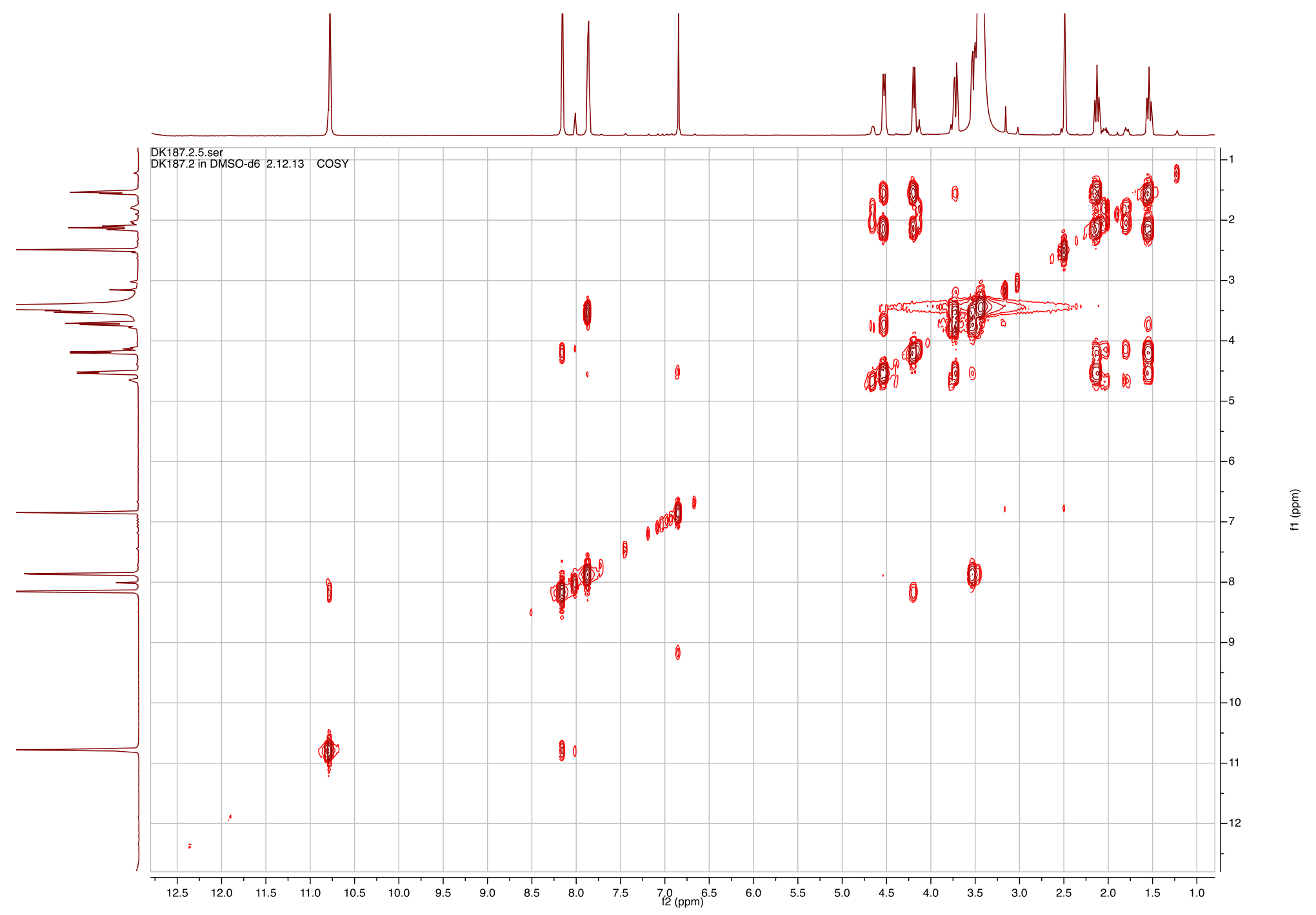


S75. ROESY Spectrum of Agesamide A in DMSO- $d_{6}$

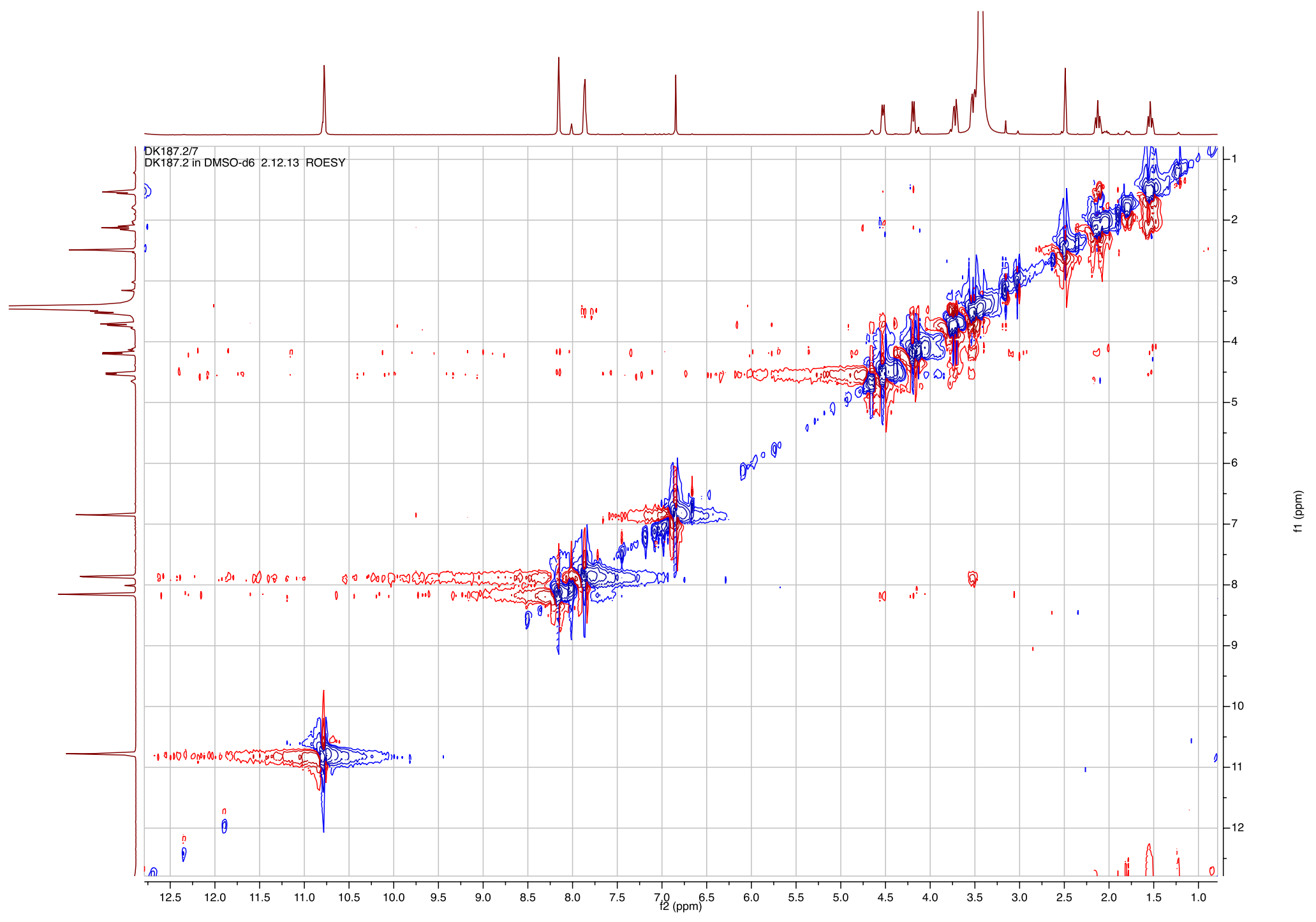


S76. Table S12. NMR data of Agesamide A in DMSO- $d_{6}{ }^{\mathrm{a}}$

\begin{tabular}{cllll}
\hline Position & $\delta_{\mathrm{C}} \mathrm{b}$ & $\delta_{\mathrm{H}}$, mult., $J(\mathrm{~Hz})$ & HMBC correlations & NOE correlations \\
\hline 2 & $106.0, \mathrm{C}$ & & $4,8 \mathrm{~b}$ & \\
3 & $99.6, \mathrm{C}$ & & 7 & \\
4 & $114.3, \mathrm{CH}$ & $6.85, \mathrm{~s}$ & 4,7 & \\
5 & $125.8, \mathrm{C}$ & & $4,8 \mathrm{~b}$ & $8 \mathrm{a}, 8 \mathrm{~b}$ \\
6 & $157.5, \mathrm{C}$ & & & $7,8 \mathrm{~b}, 9$ \\
7 & & $7.86, \mathrm{~d}(4.5)$ & $7,10 \mathrm{a}, 10 \mathrm{~b}$ & $7,8 \mathrm{a}, 9,10 \mathrm{a}$ \\
$8 \mathrm{a}$ & $40.6, \mathrm{CH} 2$ & $3.72, \mathrm{dd}(13.7,4.1)$ & $8 \mathrm{a}, 8 \mathrm{~b}, 10 \mathrm{a}, 10 \mathrm{~b}, 11,12$ \\
$\mathrm{~b}$ & & $3.52, \mathrm{ddd}(13.7,5.0,1.0)$ & & $8 \mathrm{~b}, 9,10 \mathrm{~b}, 11$ \\
9 & $51.0, \mathrm{CH}$ & $4.52, \mathrm{ddd}(11.2,4.1,3.4)$ & $7,8 \mathrm{a}, 8 \mathrm{~b}, 10 \mathrm{a}, 10 \mathrm{~b}, 11$ & $9,10 \mathrm{a}, 11,12$ \\
$10 \mathrm{a}$ & $33.9, \mathrm{CH} 2$ & $2.13, \mathrm{ddd}(14.0,11.2,3.7)$ & & $8 \mathrm{~b}, 9,10 \mathrm{a}, 10 \mathrm{~b}, 12$ \\
$\mathrm{~b}$ & & $1.54, \mathrm{ddd}(14.0,10.8,3.4)$ & & $9,10 \mathrm{~b}, 11$ \\
11 & $54.7, \mathrm{CH}$ & $4.19, \mathrm{ddd}(10.8,3.7,1.5)$ & $10 \mathrm{a}, 10 \mathrm{~b}, 12,14$ & \\
12 & & $8.15, \mathrm{~s}$ & & \\
13 & $157.9, \mathrm{C}$ & & $11,12,14$ & \\
14 & & $10.79, \mathrm{~s}$ & & $10 \mathrm{a}, 10 \mathrm{~b}, 11,12,14$ \\
15 & $175.2, \mathrm{C}$ & & & \\
\hline
\end{tabular}

${ }^{\text {a } 500.13 ~ M H z ~ f o r ~}{ }^{1} \mathrm{H}$ and $125.76 \mathrm{MHz}$ for ${ }^{13} \mathrm{C}$. ${ }^{b}$ Multiplicity and assignment from HSQC experiment. 
S77. HR ESI MS data of Agesamide A

\section{Elemental Composition Report}

Page 1

Single Mass Analysis

Tolerance $=2.0$ PPM / DBE: $\min =-1.5, \max =50.0$

Number of isotope peaks used for i-FIT $=3$

Monoisotopic Mass, Even Electron lons
1653 formula(e) evaluated with 2 results within limits (all results (up to 1000) for each mass)

$\begin{array}{llll}\text { Elements Used: } & \text { N: } 1-20 \quad \text { H: } 1-40 \quad \text { N:10 } & \text { O: 0-10 Na: 0-1 } \quad 798 r: 0-2\end{array}$

DK187.2

(6)

100

1: TOF MS ES+
$4.25 \mathrm{e}+004$

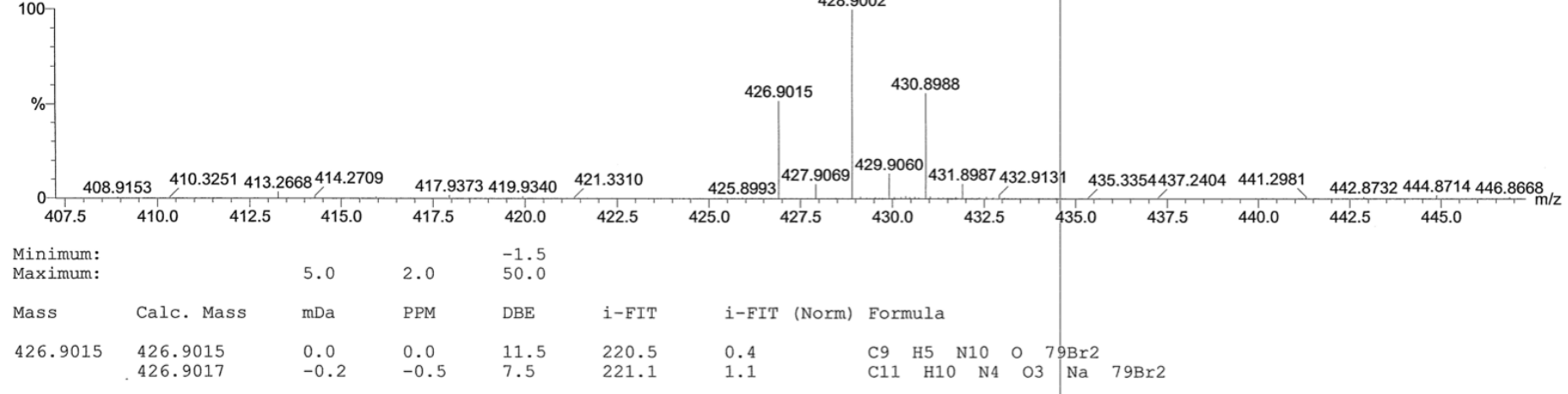


S78. Physiochemical data of Agesamide B

Agesamide B: Amorphous white solid; $[\alpha]^{25} \mathrm{D}^{-17.8}$ (c 0.55 DMSO); UV (MeOH) $\lambda_{\max }$ (log $\varepsilon$ ) $203 \mathrm{~nm}(4.16), 235 \mathrm{~nm}(3.85), 282 \mathrm{~nm}(3.87)$; ECD (c $0.124 \mathrm{mM}, \mathrm{MeCN}) \lambda_{\max }(\Delta \varepsilon) 260$ 2.00), 233 (0.39), and 217 (0.95) cm²/mol; IR (ATR Diamond) $v_{\max }$ 3049, 2361, 2342, 1769, 1718, $1649,1553 \mathrm{~cm}^{-1}$; ${ }^{1} \mathrm{H}$ and ${ }^{13} \mathrm{C}$ NMR, Table S13; HRESIMS $\mathrm{m} / z$, $426.9016[\mathrm{M}+\mathrm{Na}]^{+}$(calcd for $\mathrm{C}_{11} \mathrm{H}_{10} \mathrm{Br}_{2} \mathrm{~N}_{4} \mathrm{NaO}_{3}, 426.9017$ ). 
S79. ${ }^{1} \mathrm{H}$ NMR Spectrum of Agesamide B in DMSO- $d_{6}$

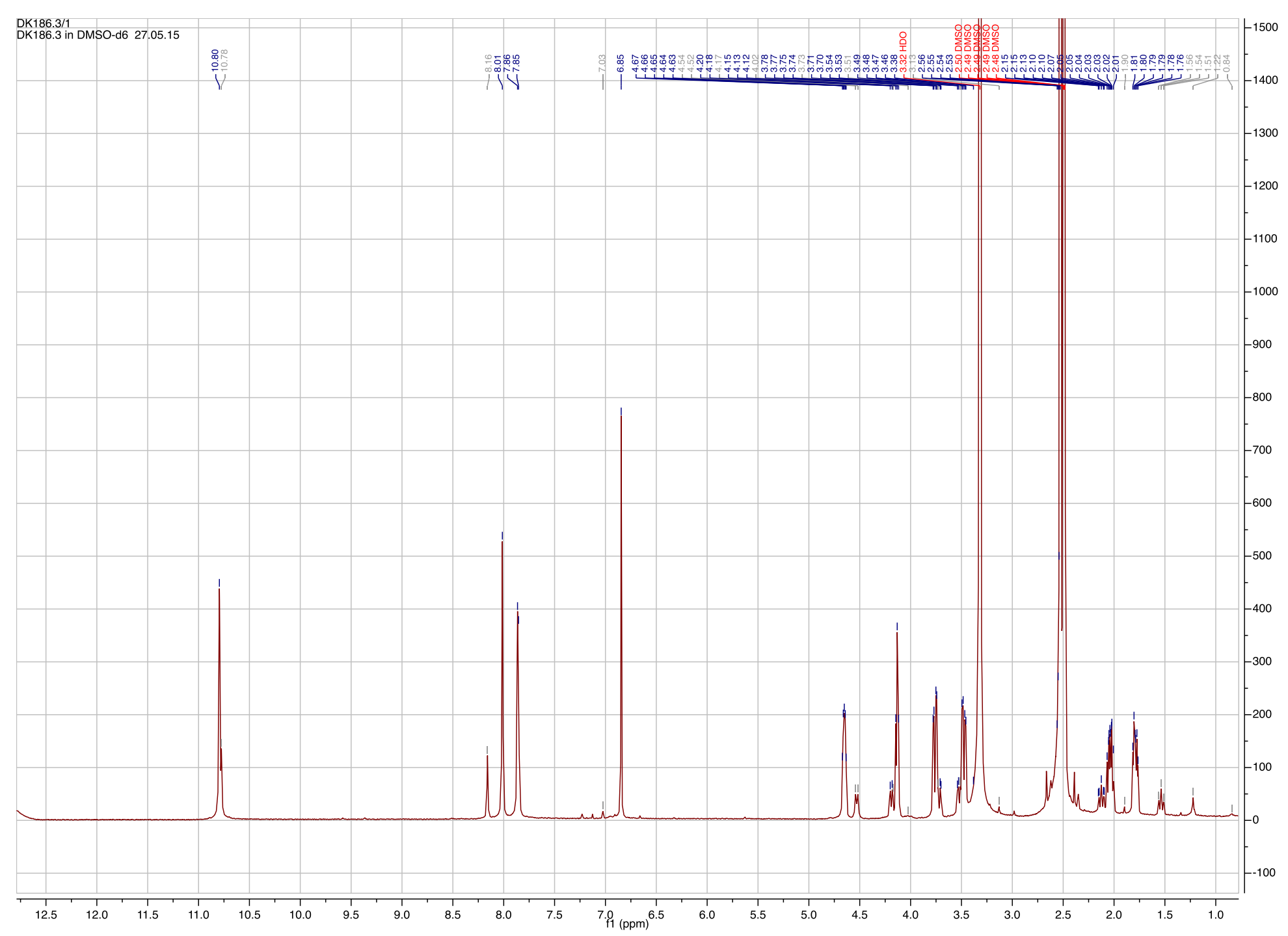


S80. ${ }^{13}$ C NMR Spectrum of Agesamide B in DMSO- $d_{6}$

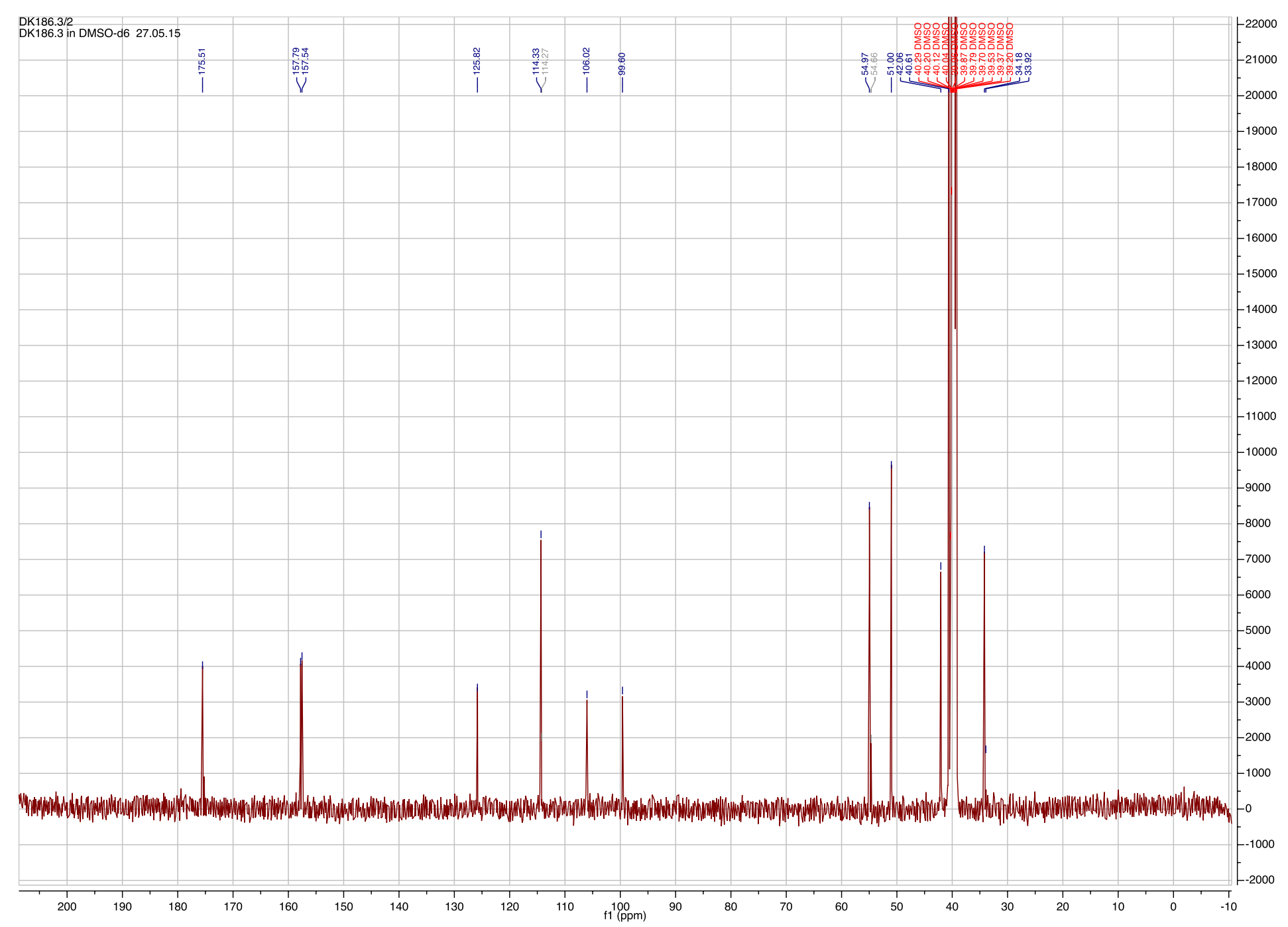


S81. HSQC Spectrum of Agesamide B in DMSO- $d_{6}$

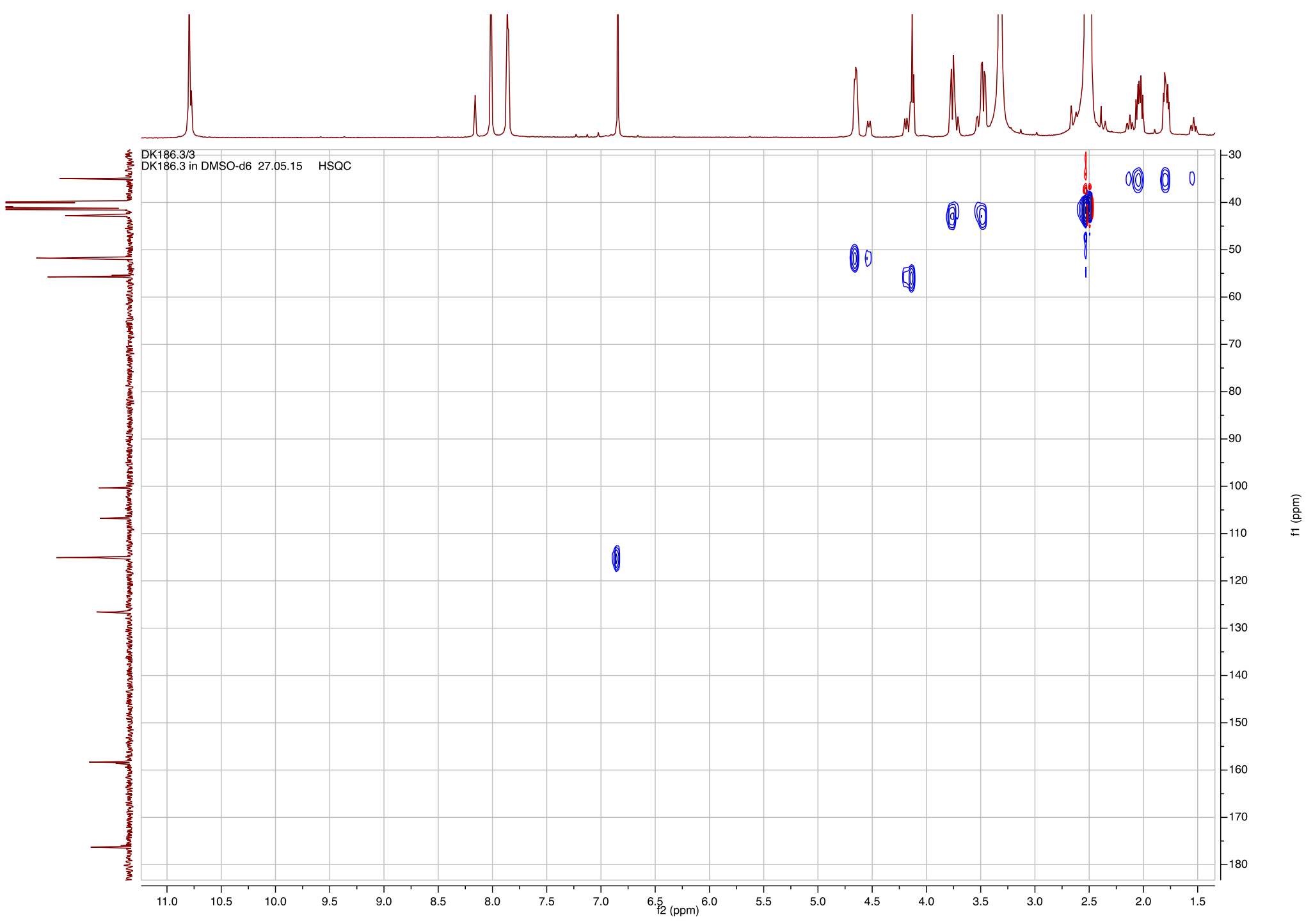


S82. HMBC Spectrum of Agesamide B in DMSO- $d_{6}$

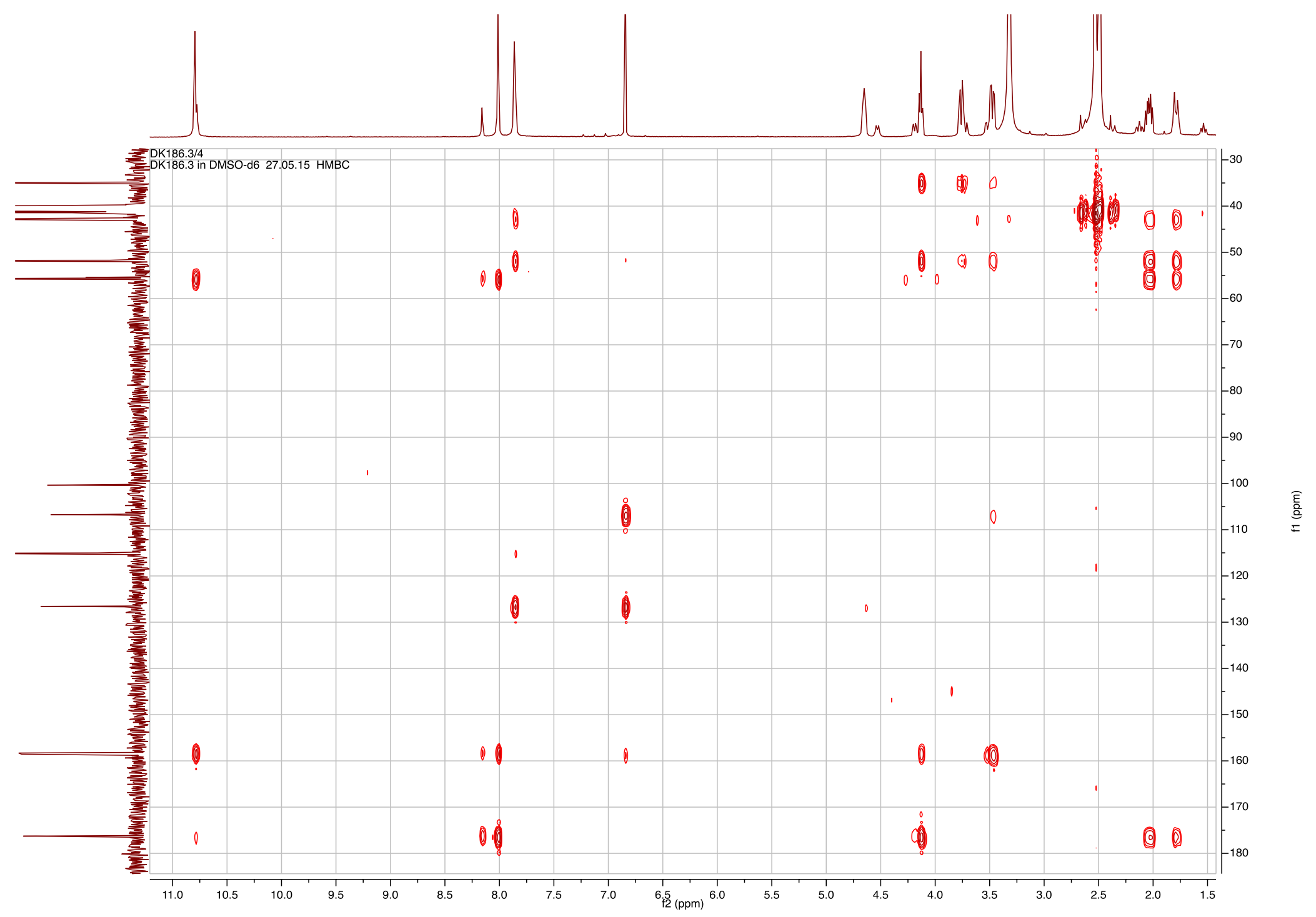


S83. COSY Spectrum of Agesamide B in DMSO- $d_{6}$

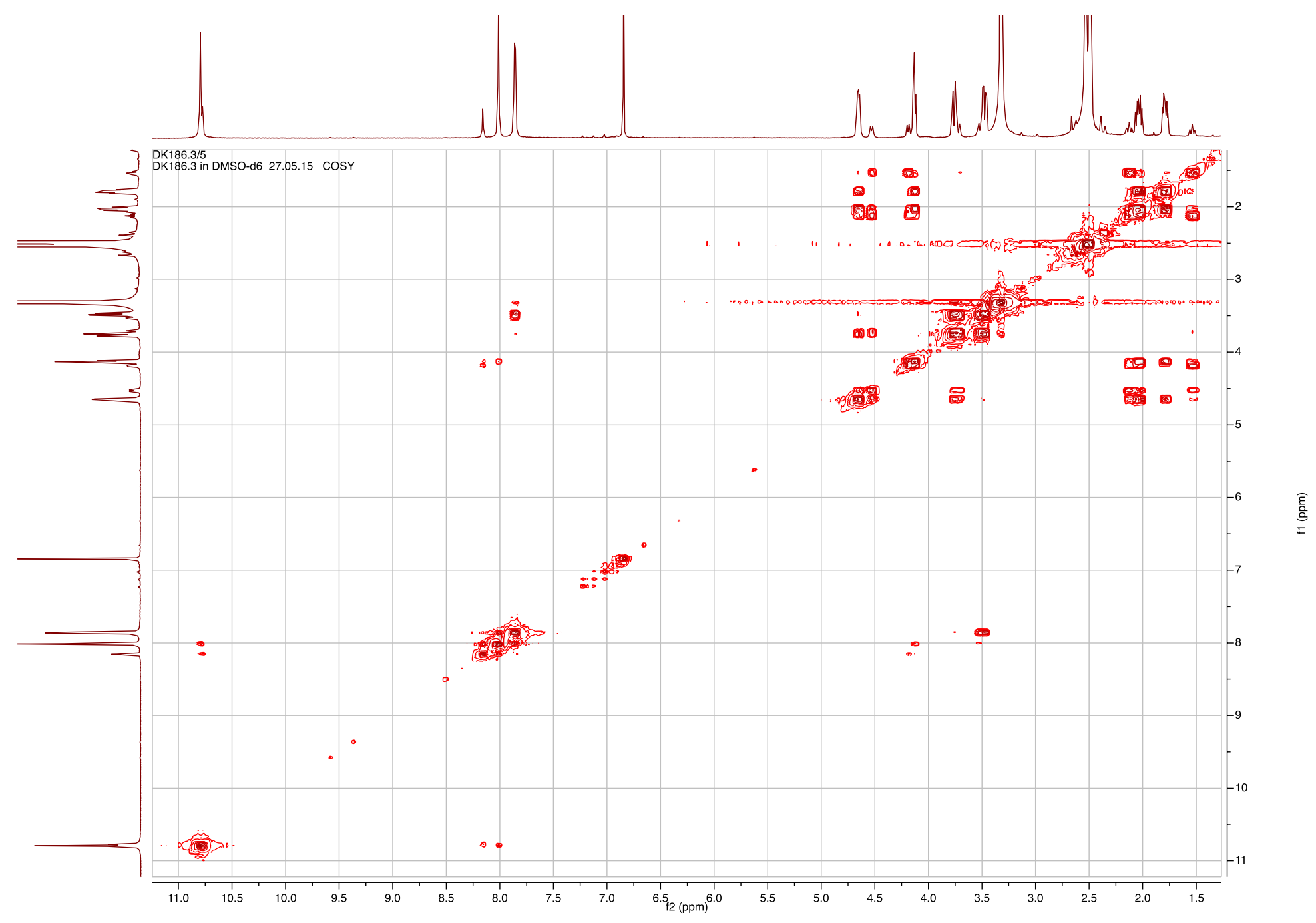


S84. ROESY Spectrum of Agesamide B in DMSO- $d_{6}$

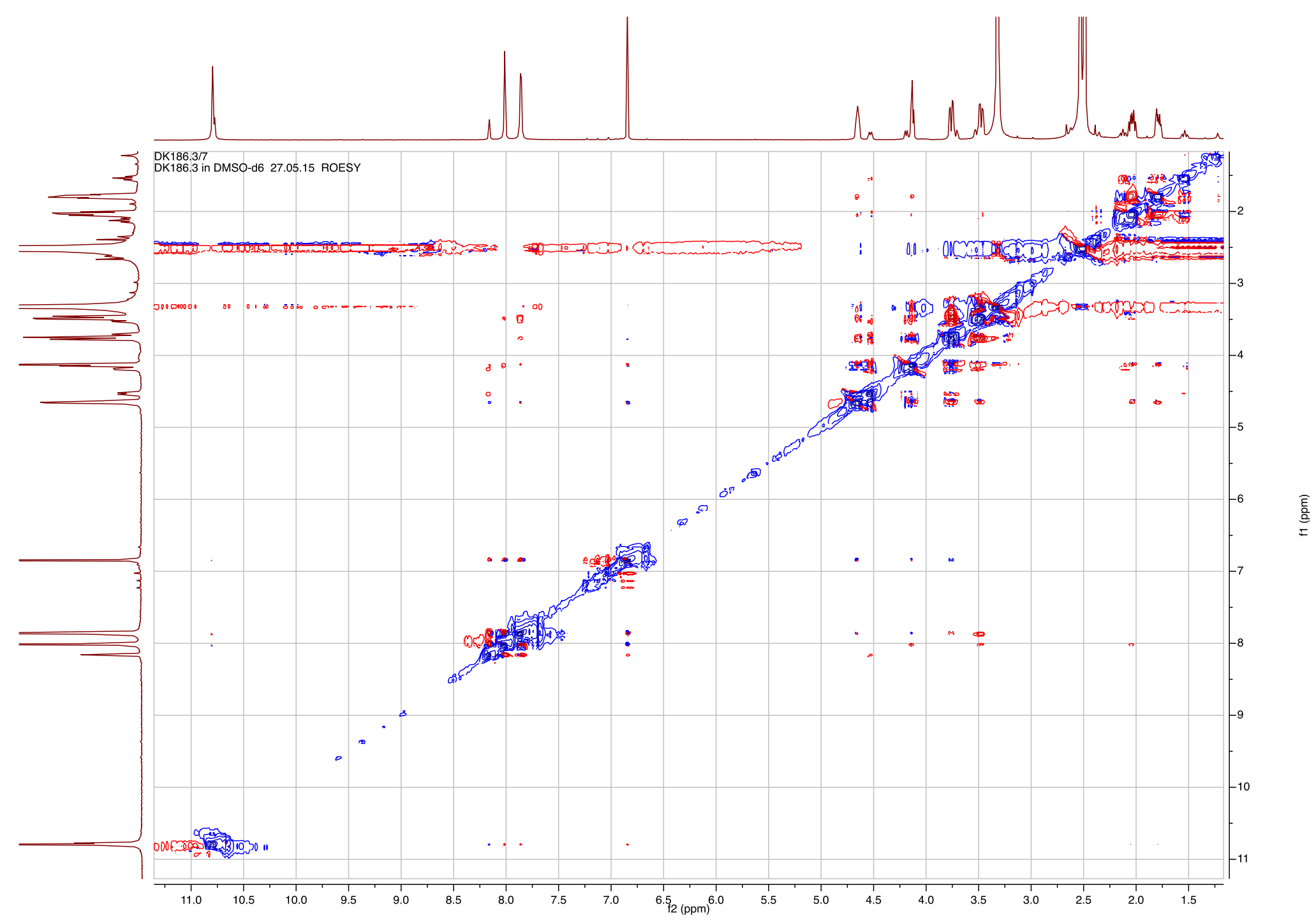


S85. Table S13. NMR data of Agesamide B in DMSO- $d_{6}{ }^{\mathrm{a}}$

\begin{tabular}{|c|c|c|c|c|}
\hline Position & $\delta_{\mathrm{cb}}$ & $\delta_{\mathrm{H}}$, mult., $J(\mathrm{~Hz})$ & HMBC correlations & NOE correlations \\
\hline 2 & $106.0, \mathrm{C}$ & & 4,9 & \\
\hline 3 & $99.6, \mathrm{C}$ & & & \\
\hline 4 & $114.3, \mathrm{CH}$ & $6.85, \mathrm{~s}$ & & \\
\hline 5 & $125.8, \mathrm{C}$ & & $4,7,9$ & \\
\hline 6 & $157.5, \mathrm{C}$ & & $4,8 b$ & \\
\hline 7 & & $7.85, \mathrm{~d}(4.4)$ & & $8 \mathrm{a}, 8 \mathrm{~b}$ \\
\hline $\begin{array}{r}8 \mathrm{a} \\
\mathrm{b}\end{array}$ & $42.1, \mathrm{CH}_{2}$ & $\begin{array}{l}3.76, \text { dd }(14.1,4.6) \\
3.47, \text { dd }(14.1,5.0)\end{array}$ & $7,10 \mathrm{a}, 10 \mathrm{~b}$ & $\begin{array}{l}7,8 \mathrm{~b}, 9 \\
7,8 \mathrm{a}, 9,10 \mathrm{a}, 11,12\end{array}$ \\
\hline 9 & $51.0, \mathrm{CH}$ & 4.65, ddd $(9.4,4.9,4.0)$ & $7,8 \mathrm{a}, 8 \mathrm{~b}, 10 \mathrm{a}, 10 \mathrm{~b}, 11$ & $8 \mathrm{a}, 8 \mathrm{~b}, 10 \mathrm{a}, 10 \mathrm{~b}, 11,12$ \\
\hline $\begin{array}{r}10 \mathrm{a} \\
\mathrm{b}\end{array}$ & $34.1, \mathrm{CH}_{2}$ & $\begin{array}{l}2.04, \operatorname{ddd}(14.0,9.4,6.8) \\
1.80, \operatorname{ddd}(14.0,6.8,4.0)\end{array}$ & $8 \mathrm{a}, 8 \mathrm{~b}, 11$ & $\begin{array}{l}8 \mathrm{~b}, 9,10 \mathrm{~b}, 11,12 \\
9,10 \mathrm{a}, 11\end{array}$ \\
\hline 11 & $55.0, \mathrm{CH}$ & $4.14, \mathrm{t}(6.8)$ & $10 \mathrm{a}, 10 \mathrm{~b}, 12,14$ & $8 \mathrm{~b}, 9,10 \mathrm{a}, 10 \mathrm{~b}, 12$ \\
\hline 12 & & $8.00, \mathrm{~s}$ & & $8 \mathrm{~b}, 9,10 \mathrm{a}, 11$ \\
\hline 13 & $157.8, \mathrm{C}$ & & $10 \mathrm{a}, 10 \mathrm{~b}, 11,12,14$ & \\
\hline 14 & & $10.78, \mathrm{~s}$ & & \\
\hline 15 & $175.5, \mathrm{C}$ & & $10 \mathrm{a}, 10 \mathrm{~b}, 12,14$ & \\
\hline
\end{tabular}

$\mathrm{a}_{500.13 \mathrm{MHz} \text { for }}{ }^{1} \mathrm{H}$ and $125.76 \mathrm{MHz}$ for ${ }^{13} \mathrm{C}$. ${ }^{\mathrm{b}}$ Multiplicity and assignment from HSQC experiment. 
S86. HR ESI MS data of Agesamide B

\section{Elemental Composition Report}

Page 1

Single Mass Analysis

Tolerance $=2.0$ PPM $/$ DBE: $\min =-1.5, \max =50.0$

Element prediction: Off
Number of isotope peaks used for i-FIT $=3$

Monoisotopic Mass, Even Electron lons

2 results within limits (all results (up to 1000) for each mass)

$\begin{array}{lllll}\text { Elements Us: } & \text { H: } 1-40 \quad \mathrm{~N}: 0-10 & \text { O: 0-10 Na: 0-1 } & \text { 79Br: 0-2 }\end{array}$

DK186.3

(92:101)

Dmitri Kovalerchik

100

428.9017

1: TOF MS ES+

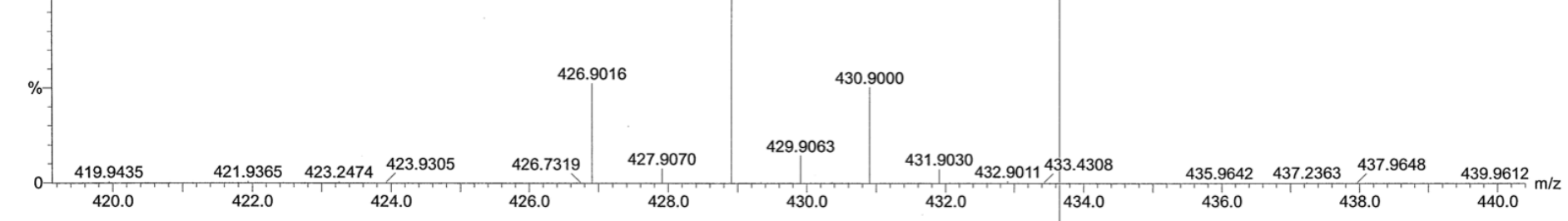

Minimum:

4232474423.9305

434.0

436.0

440.

$5.0-2.0-50.5$

0.1

-1.5
50.0

$426.9016 \quad 426.901$
426.901

$\begin{array}{llll}0.1 & 0.2 & 11.5 & 413.0 \\ -0.1 & -0.2 & 7.5 & 413.5\end{array}$

i-FIT (Norm) Formula
0.5
1.0

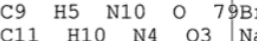
$\mathrm{Na} 79 \mathrm{Br} 2$ 
S87. Physiochemical data of Agesamine A

Agesamine A: Amorphous white solid; $[\alpha]^{25} \mathrm{D}^{-0.7}(c 0.84 \mathrm{MeOH})$; UV (MeOH) $\lambda_{\max }(\log \varepsilon) 203 \mathrm{~nm}(4.02), 227 \mathrm{~nm}(3.79), 282 \mathrm{~nm}(3.55)$; ECD (c $0.124 \mathrm{mM}, \mathrm{MeCN}) \lambda_{\max }(\Delta \varepsilon) 259$ (0.05), 243 (-0.25), 232 (-0.22), and $214(0.34) \mathrm{cm}^{2} / \mathrm{mol}$; IR (ATR Diamond) $v_{\max } 3222,2360,2341$, 1716, 1668, $1551 \mathrm{~cm}^{-1} ;{ }^{1} \mathrm{H}$ and ${ }^{13} \mathrm{C}$ NMR, Table S14; HRESIMS $\mathrm{m} / z, 403.9360[\mathrm{M}+\mathrm{H}]^{+}\left(\right.$calcd for $\mathrm{C}_{11} \mathrm{H}_{12} \mathrm{Br}_{2} \mathrm{~N}_{5} \mathrm{O}_{2}, 403.9358$ ). 
S88. ${ }^{1} \mathrm{H}$ NMR Spectrum of Agesamine A in DMSO- $d_{6}$

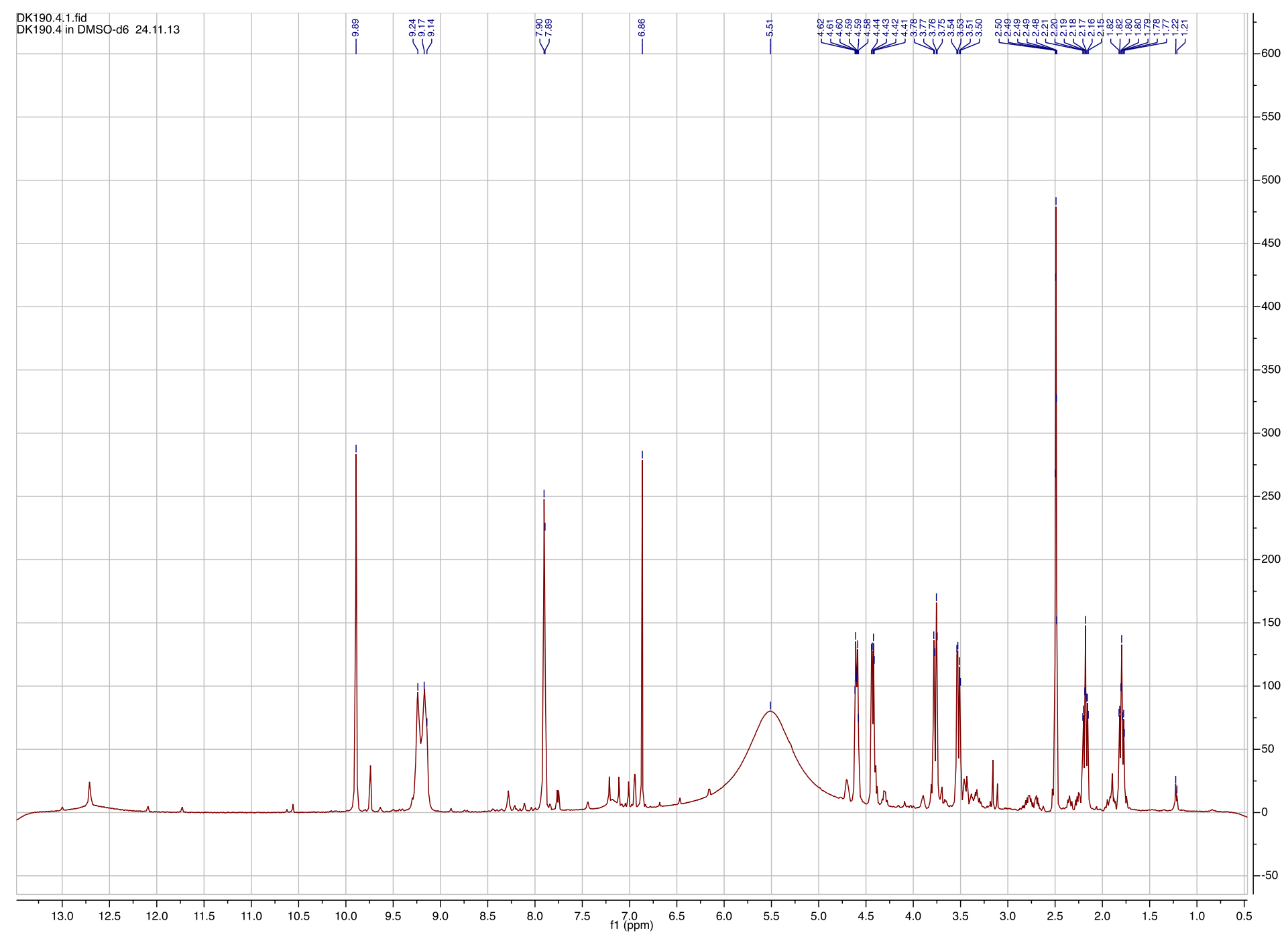


S89. ${ }^{13} \mathrm{C}$ NMR Spectrum of Agesamine A in DMSO- $d_{6}$

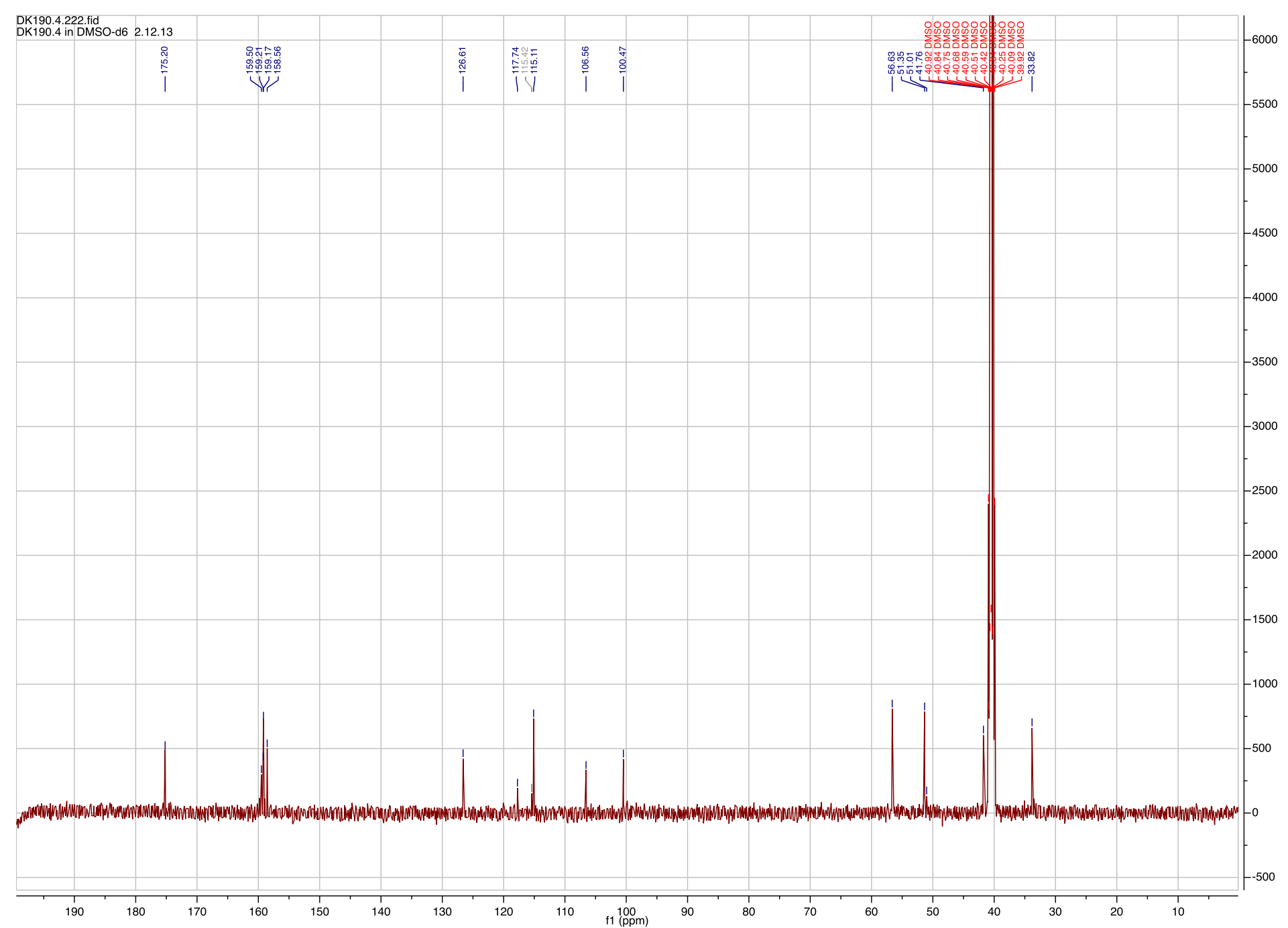


S90. HSQC Spectrum of Agesamine A in DMSO- $d_{6}$

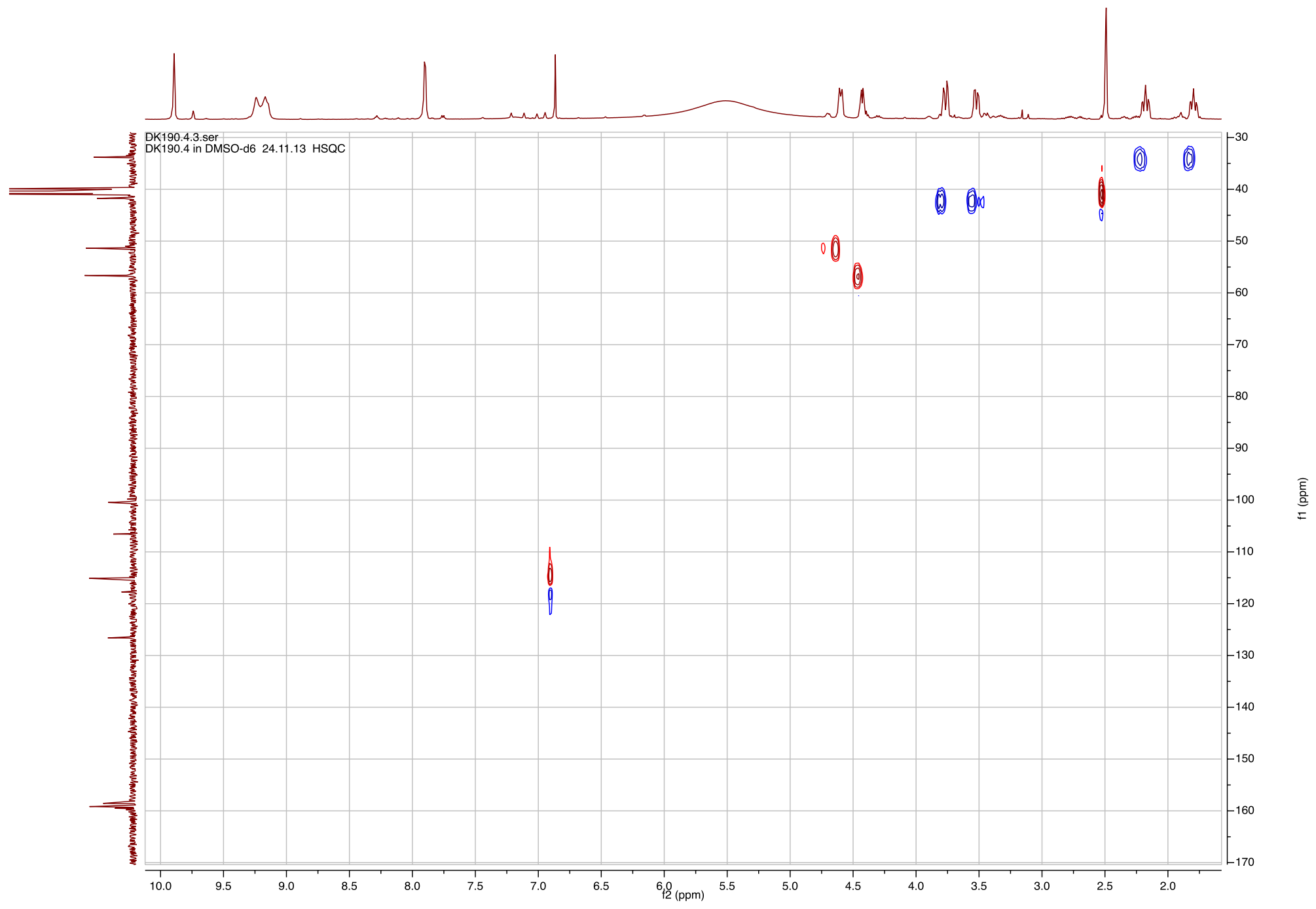


S91. HMBC Spectrum of Agesamine A in DMSO- $d_{6}$

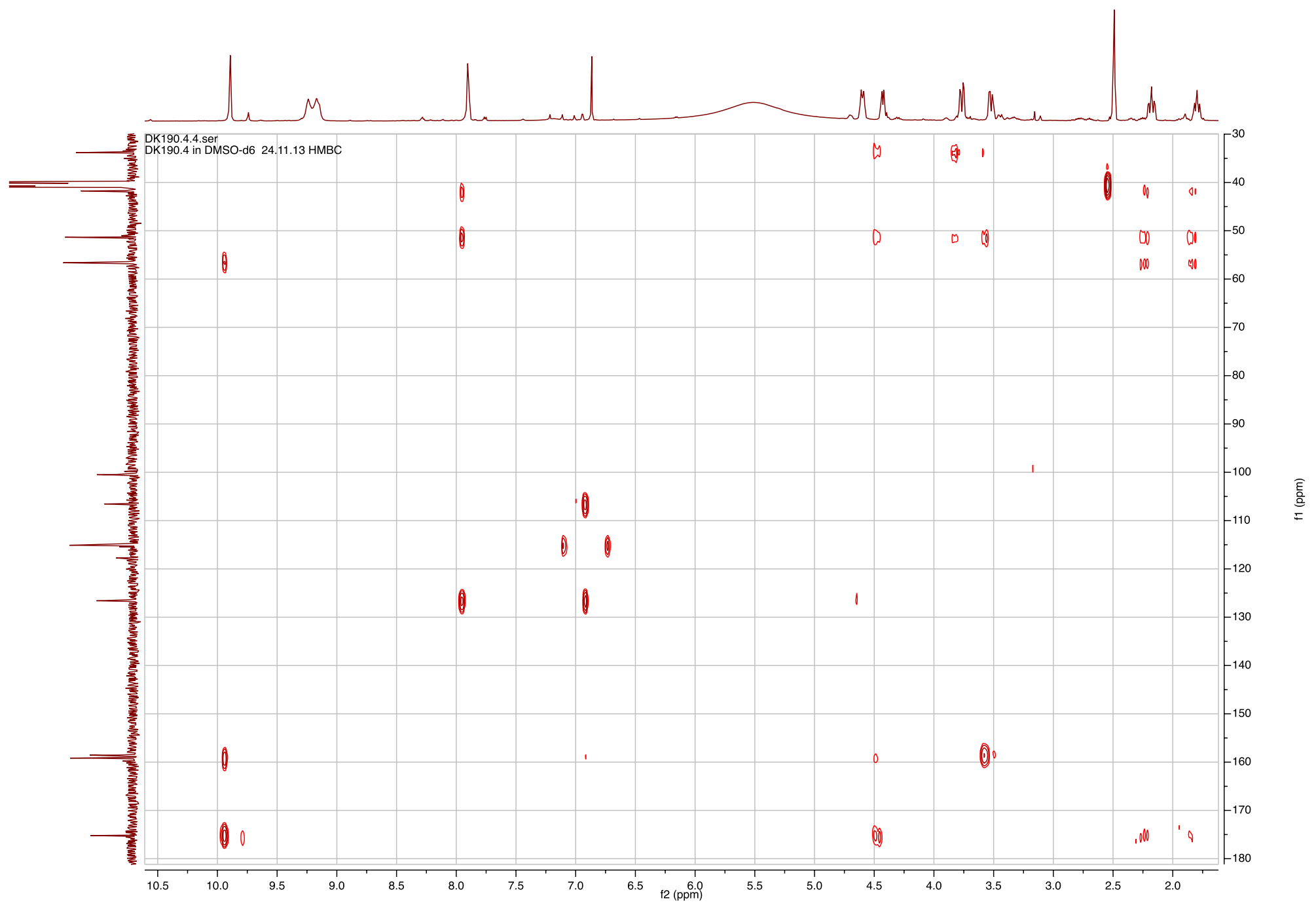


S92. COSY Spectrum of Agesamine A in DMSO- $d_{6}$

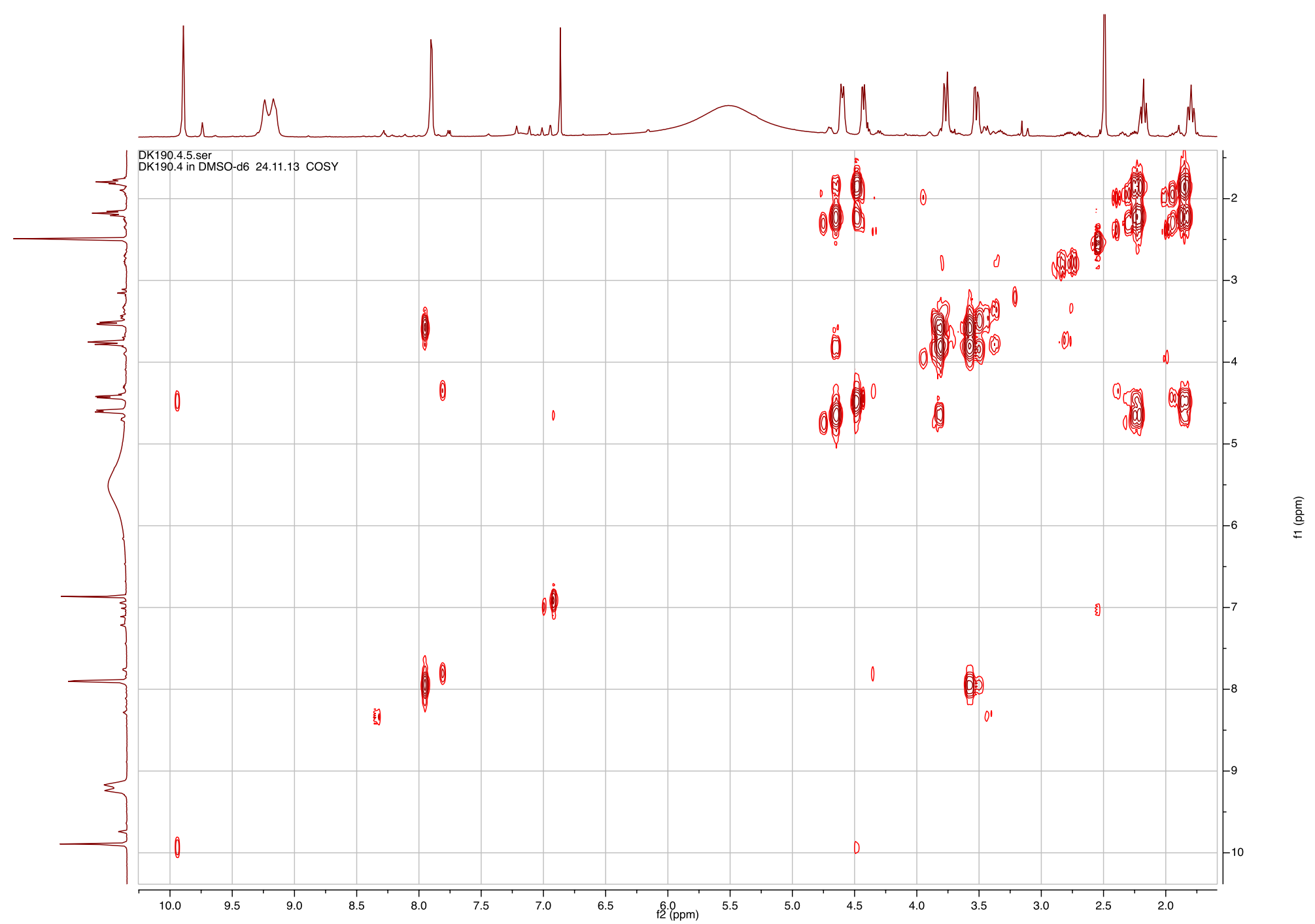


S93. Difference-NOE Spectra of Agesamine A in DMSO- $d_{6}$

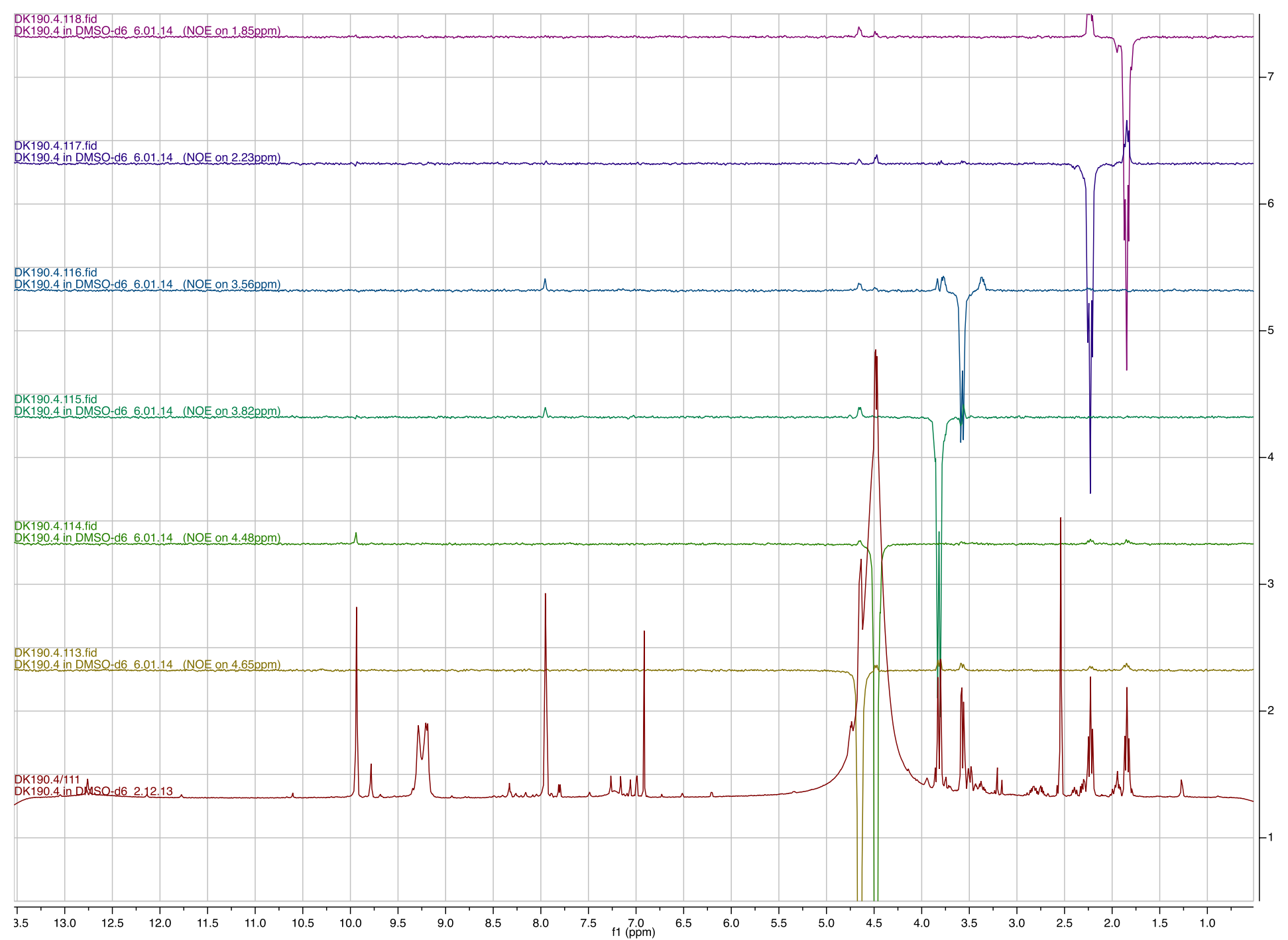


S94. Table S14. NMR data of Agesamine A in DMSO- $d_{6}{ }^{\mathrm{a}}$

\begin{tabular}{|c|c|c|c|c|}
\hline Position & $\delta_{\mathrm{C}}^{\mathrm{b}}$ & $\delta_{\mathrm{H}}$, mult., $J(\mathrm{~Hz})$ & HMBC correlations & NOE correlations \\
\hline 2 & $105.8, \mathrm{C}$ & & 4,9 & \\
\hline 3 & $99.7, \mathrm{C}$ & & & \\
\hline 4 & $114.4, \mathrm{CH}$ & $6.86, \mathrm{~s}$ & & \\
\hline 5 & $125.9, \mathrm{C}$ & & $4,7,9$ & \\
\hline 6 & $157.8, \mathrm{C}$ & & $4,8 b$ & \\
\hline 7 & & $7.90, \mathrm{~d}(5.0)$ & & $8 \mathrm{a}, 8 \mathrm{~b}$ \\
\hline $8 \mathrm{a}$ & $41.0, \mathrm{CH}_{2}$ & 3.76 , dd $(13.5,3.5)$ & $7,10 \mathrm{a}, 10 \mathrm{~b}$ & $7,8 b, 9$ \\
\hline $\mathrm{b}$ & & $3.51, \mathrm{dd}(13.5,5.0)$ & & $7,8 \mathrm{a}, 9$ \\
\hline 9 & $50.6, \mathrm{CH}$ & 4.59, ddd $(10.7,3.7,3.5)$ & $7,8 \mathrm{a}, 8 \mathrm{~b}, 10 \mathrm{a}, 10 \mathrm{~b}, 11$ & $8 \mathrm{a}, 8 \mathrm{~b}, 10 \mathrm{a}, 10 \mathrm{~b}, 11$ \\
\hline $10 \mathrm{a}$ & $33.1, \mathrm{CH}_{2}$ & 2.18, ddd $(14.1,10.7,4.0)$ & $8 \mathrm{a}, 8 \mathrm{~b}, 11$ & $9,10 b, 11$ \\
\hline $\mathrm{b}$ & & 1.80, ddd $(14.1,10.4,3.7)$ & & $9,10 \mathrm{a}, 11$ \\
\hline 11 & $55.9, \mathrm{CH}$ & 4.42 , ddd $(10.4,4.0,1.0)$ & $10 \mathrm{a}, 10 \mathrm{~b}, 12$ & $9,10 \mathrm{a}, 10 \mathrm{~b}, 12$ \\
\hline 12 & & $9.89, \mathrm{~s}$ & & $11,13-\mathrm{NH}$ \\
\hline 13 & $158.5, \mathrm{C}$ & & 11,12 & \\
\hline $13-\mathrm{NH}$ & & 9.16, brs & & 12,14 \\
\hline 14 & & 9.24, brs & & $13-\mathrm{NH}$ \\
\hline 15 & $174.5, \mathrm{C}$ & & $10 \mathrm{a}, 10 \mathrm{~b}, 11,12$ & \\
\hline
\end{tabular}

${ }^{\mathrm{a}} 500.13 \mathrm{MHz}$ for ${ }^{1} \mathrm{H}$ and $125.76 \mathrm{MHz}$ for ${ }^{13} \mathrm{C} .{ }^{\mathrm{b}}$ Multiplicity and assignment from HSQC experiment. 
S95. HR ESI MS data of Agesamine A

Elemental Composition Report

Page 1

\section{Single Mass Analysis}

Tolerance $=5.0 \mathrm{mDa} /$ DBE: $\min =-1.5, \max =50.0$

Element prediction: Off
Number of isotope peaks used for $\mathrm{i}-\mathrm{FIT}=3$

Monoisotopic Mass, Even Electron lons

233 formula(e) evaluated with 6 results within limits (up to 4 closest results for each mass)

$\begin{array}{lllll}\text { Elements Used: } & \text { N: } 0-10 & \text { O: 0-10 Na: 0-1 } & \text { Br: } 2-2\end{array}$

DK190.4
carmeli906 78 (3.436) $\mathrm{Cm}$ (78)

Dimitry Kovalerchik

1: TOF MS ES+

100

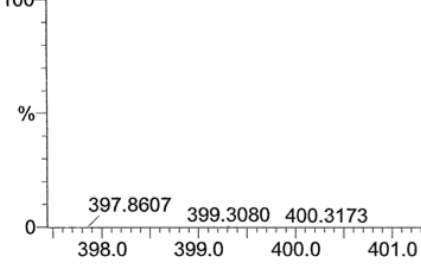

405.9342

Minimum:

Mass

$\begin{array}{lll}5.0 & 10.0 & -1.5 \\ 50.0\end{array}$

403.9360

$\begin{array}{lll} & & \\ & & \end{array}$

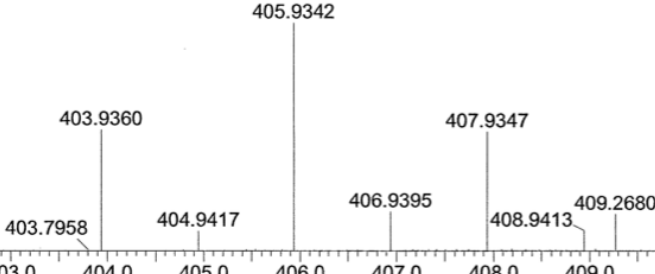

$410.2722 \quad 413.2711$ 414.2719 415.2684

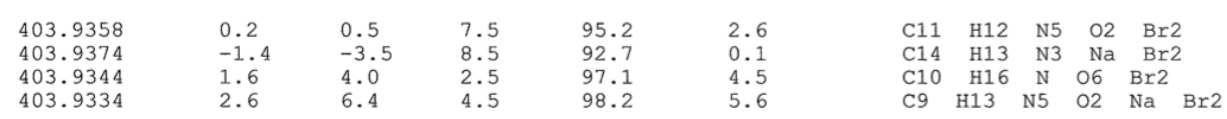


S96. Physiochemical data of Agesamine B

Agesamine B: Amorphous white solid; $[\alpha]^{25} \mathrm{D}-11.4(c 0.49, \mathrm{MeOH}) ; \mathrm{UV}(\mathrm{MeOH}) \lambda_{\max }(\log \varepsilon) 203 \mathrm{~nm}(4.21), 228 \mathrm{~nm}(3.98), 282 \mathrm{~nm}(3.72)$; ECD (c $0.124 \mathrm{mM}, \mathrm{MeCN}) \lambda_{\max }(\Delta \varepsilon) 255$ (1.20), 233 (0.52), and 217 (1.05) $\mathrm{cm}^{2} / \mathrm{mol}$; IR (ATR Diamond) $v_{\max } 3219,2360,2341,1776,1715$, 1652, $1550 \mathrm{~cm}^{-1} ;{ }^{1} \mathrm{H}$ and ${ }^{13} \mathrm{C}$ NMR, Table S15; HRESIMS $m / z$ 425.9174 [M+Na] ${ }^{+}$(calcd for $\mathrm{C}_{11} \mathrm{H}_{12} \mathrm{Br}_{2} \mathrm{~N}_{5} \mathrm{NaO}_{2}, 425.9172$ ). 
S97. ${ }^{1} \mathrm{H}$ NMR Spectrum of Agesamine B in DMSO- $d_{6}$

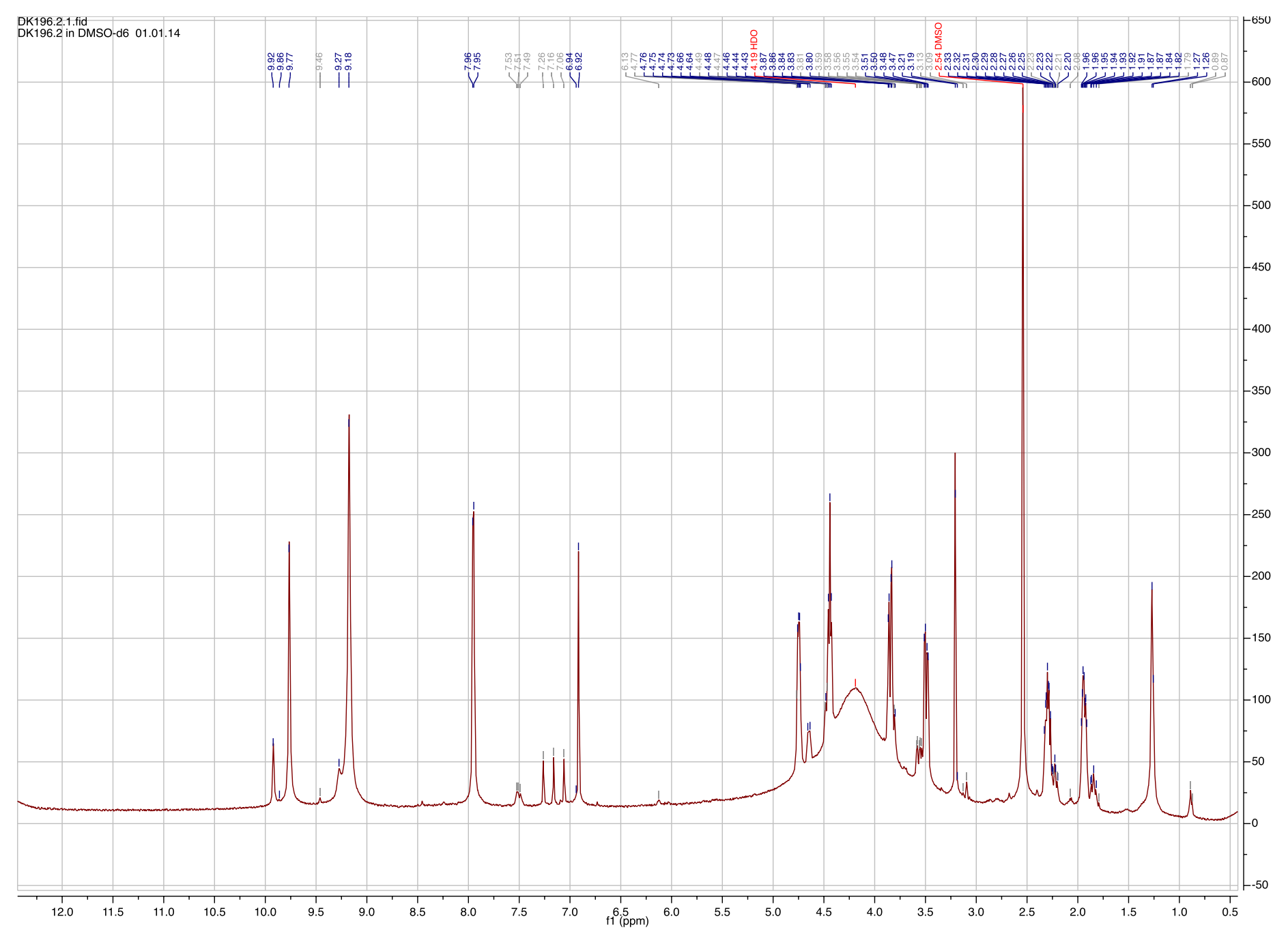


S98. ${ }^{13} \mathrm{C}$ NMR Spectrum of Agesamine B in DMSO- $d_{6}$

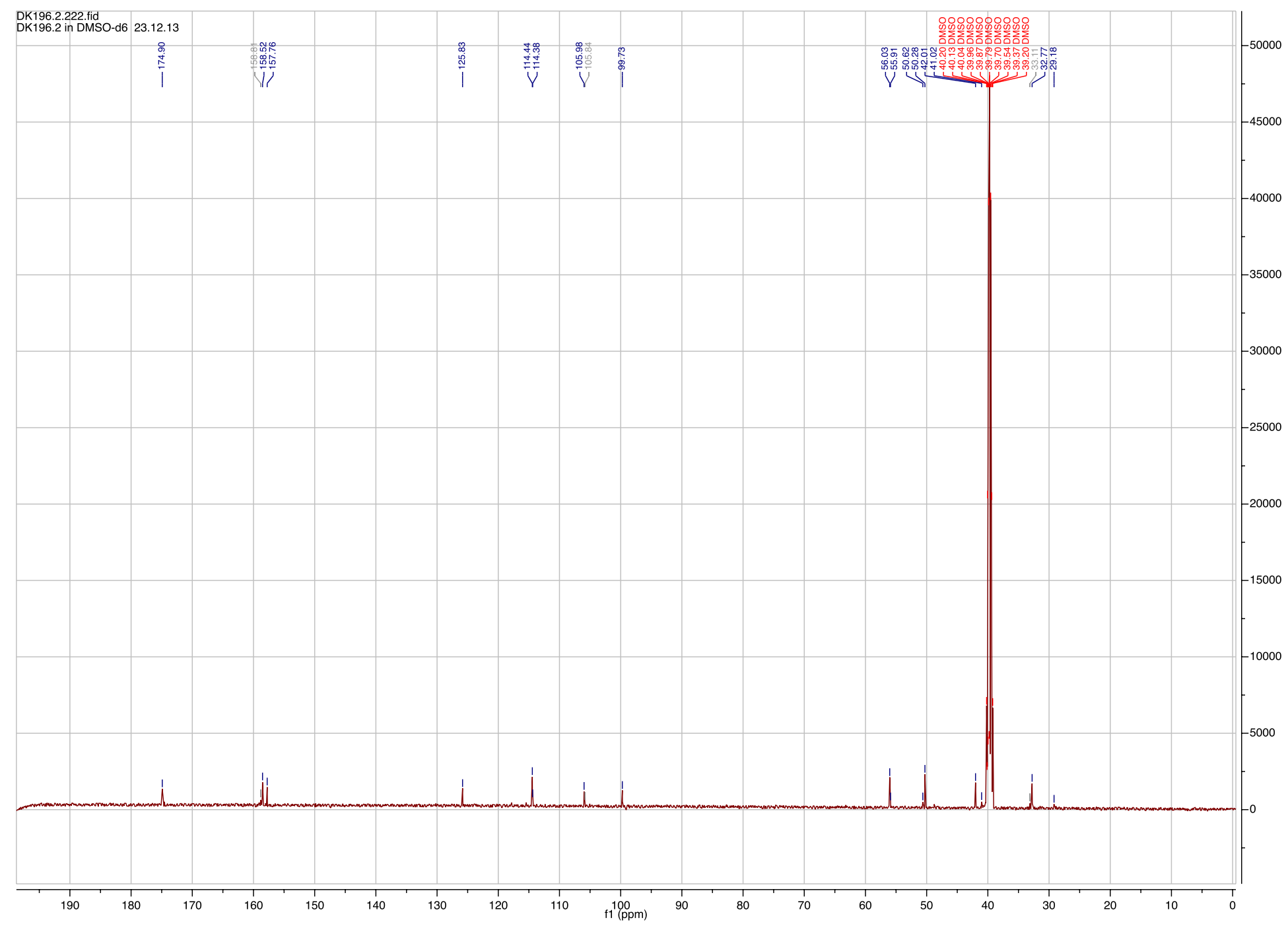


S99. HSQC Spectrum of Agesamine B in DMSO- $d_{6}$

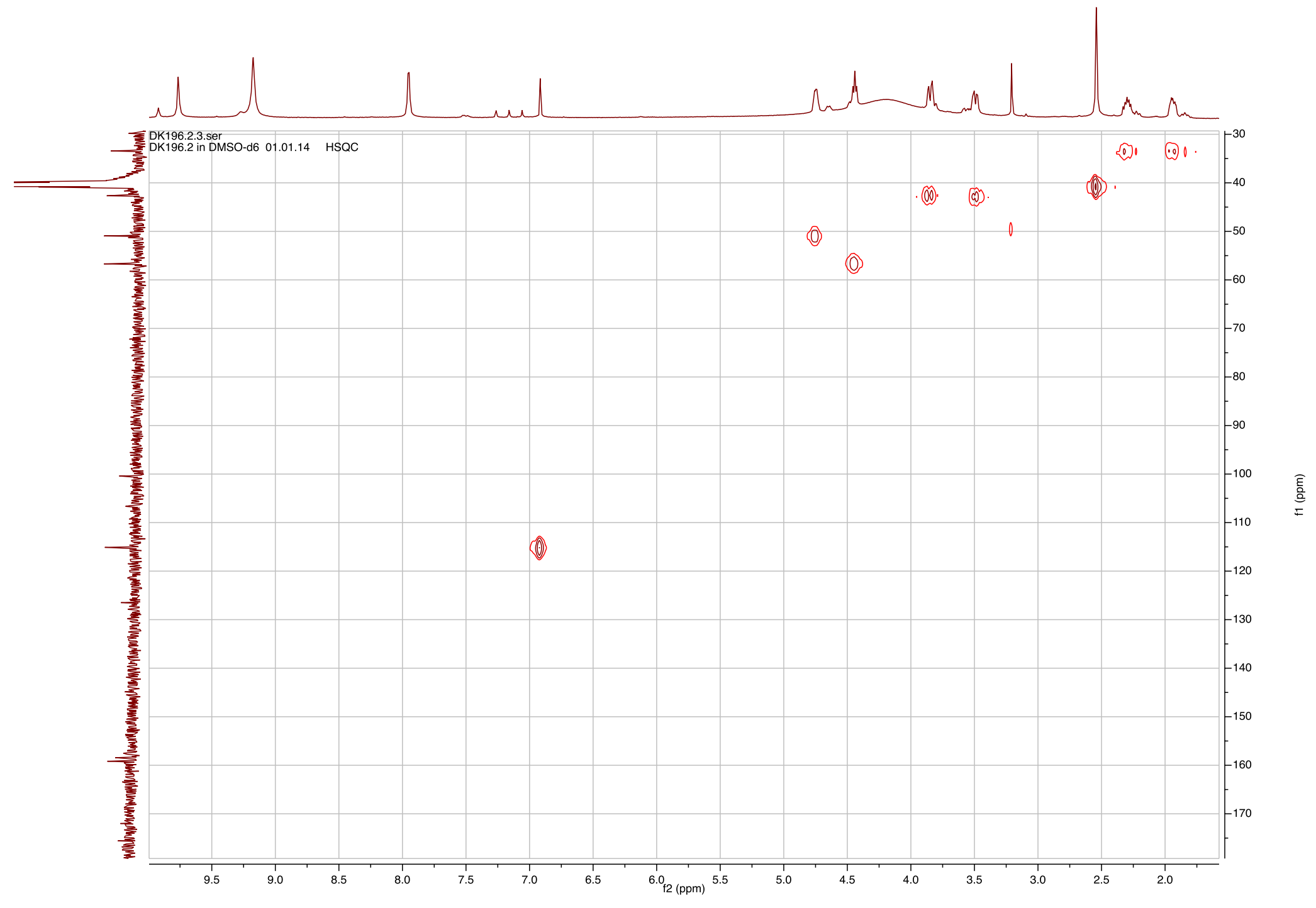


S100. HMBC Spectrum of Agesamine B in DMSO- $d_{6}$

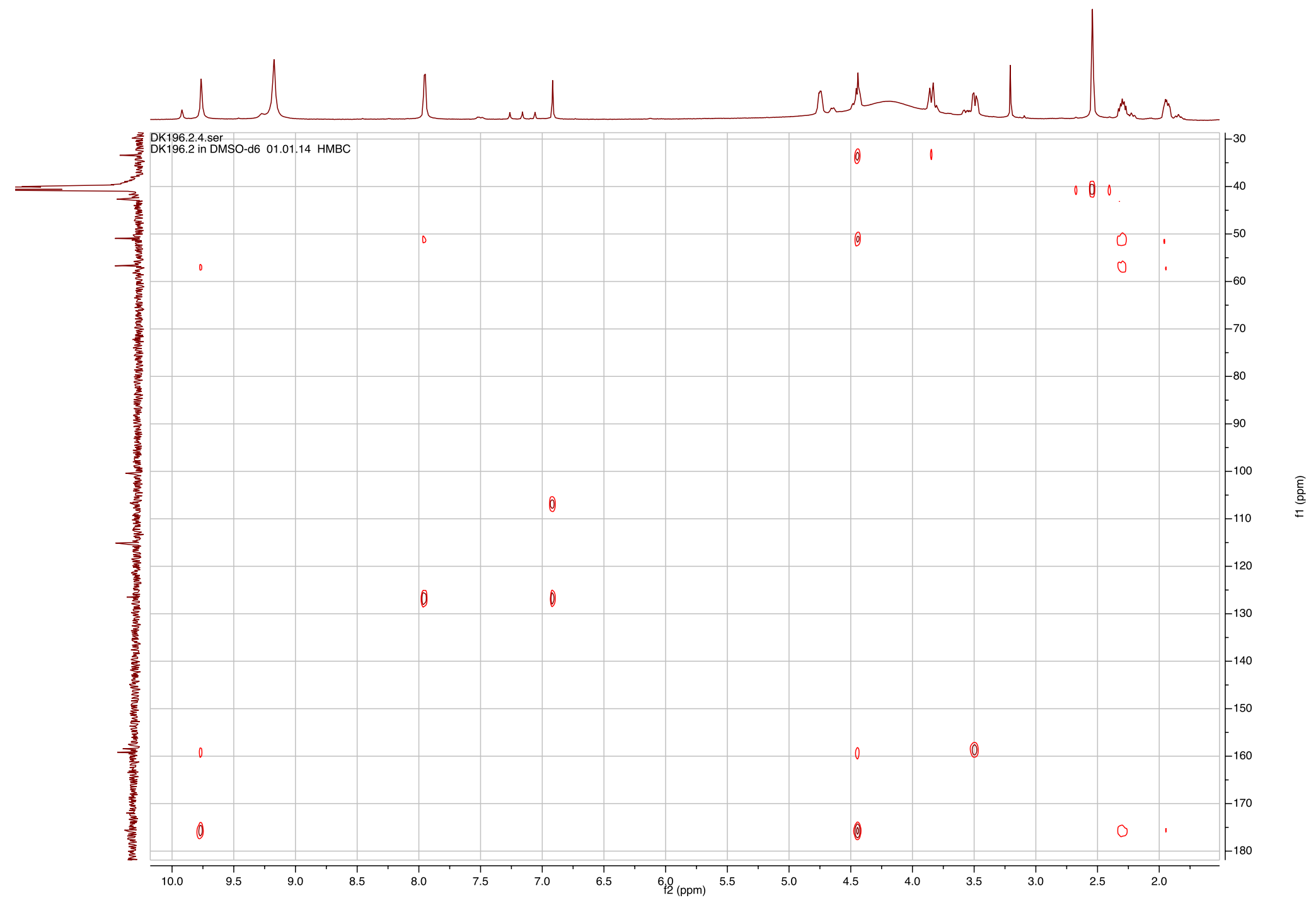


S101. COSY Spectrum of Agesamine B in DMSO- $d_{6}$

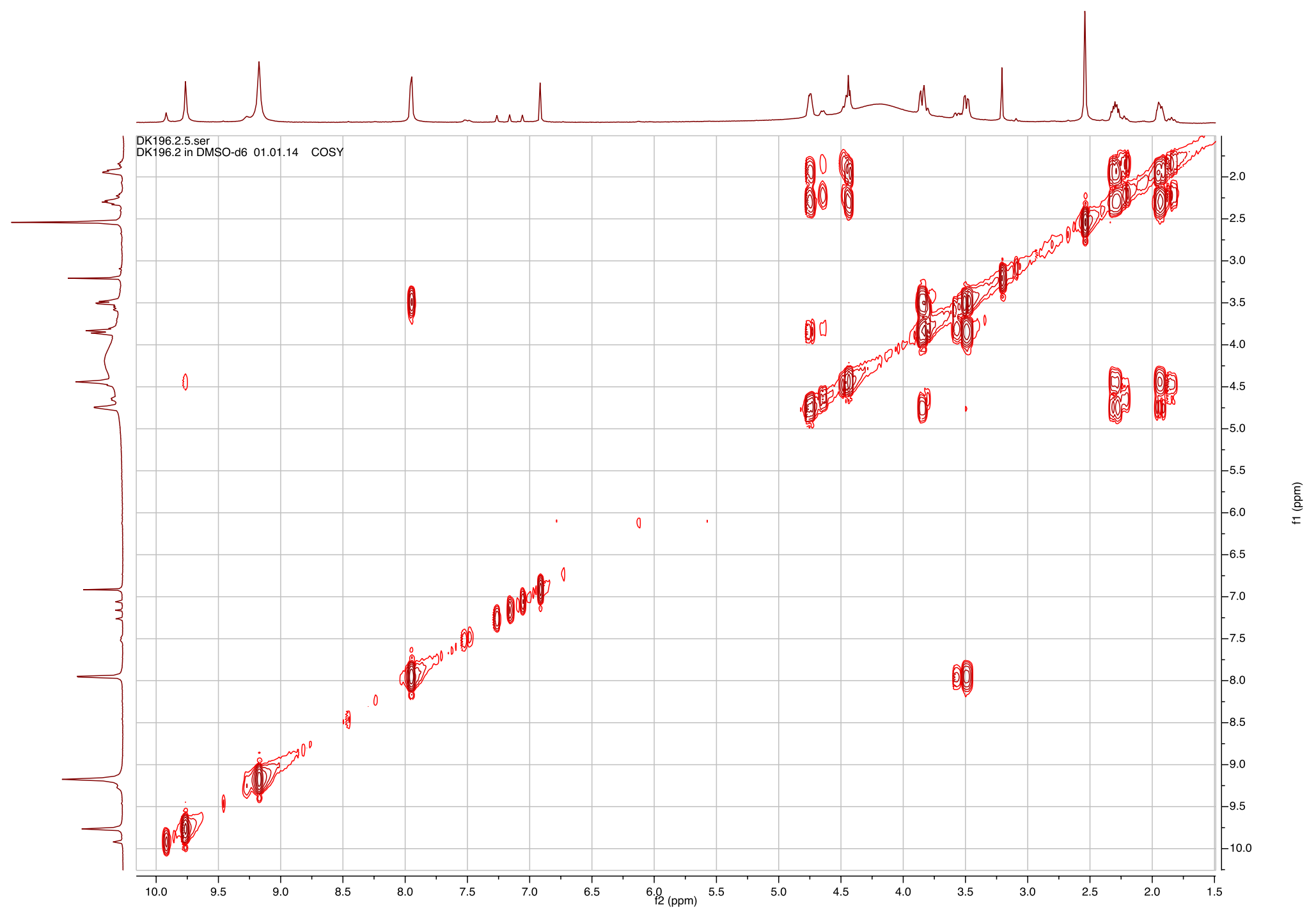


S102. Difference-NOE Spectra of Agesamine B in DMSO- $d_{6}$

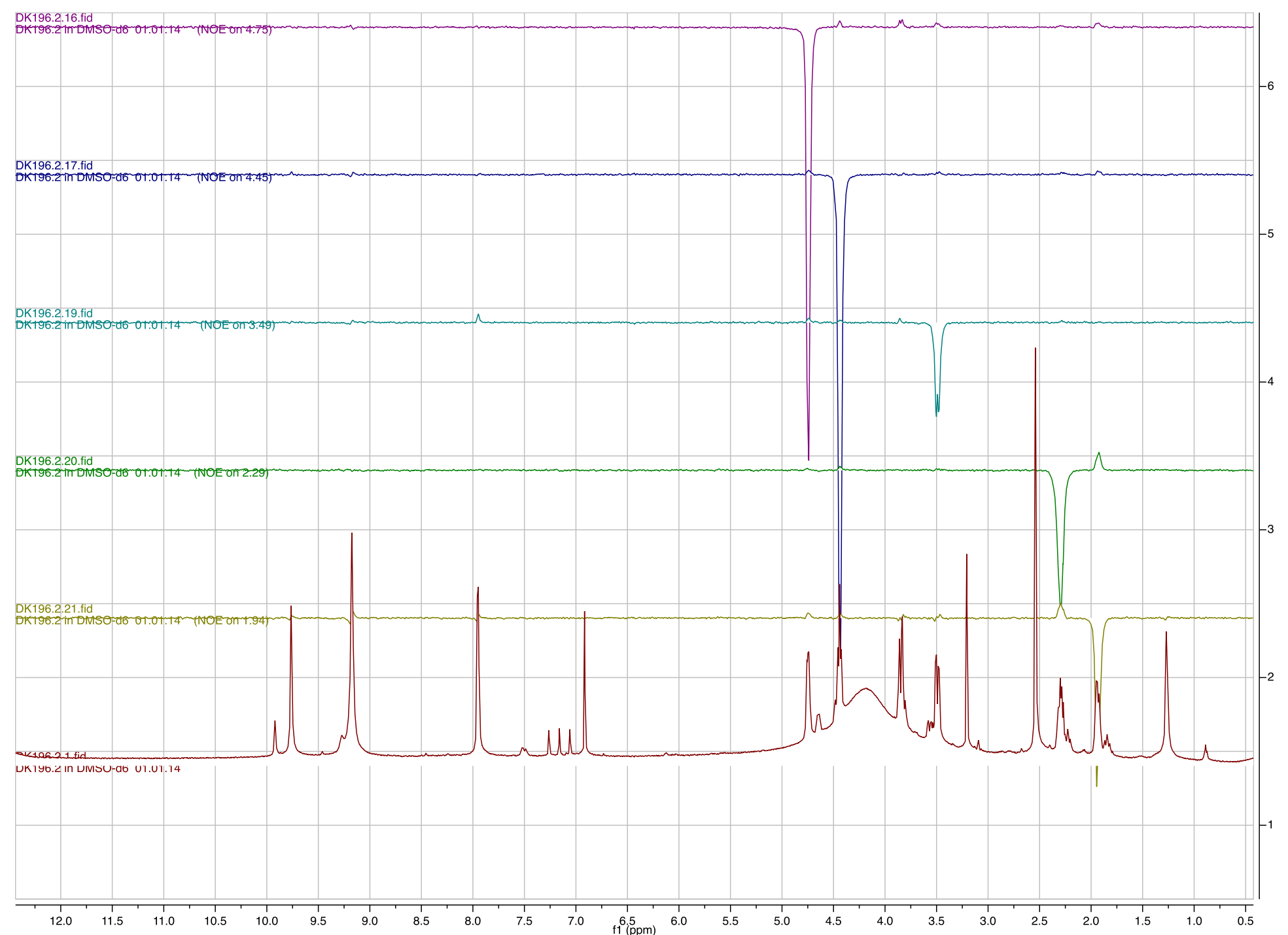


S103. Table S15. NMR data of Agesamine B in DMSO- $d_{6}{ }^{\mathrm{a}}$

\begin{tabular}{lllll}
\hline Position & $\delta_{\mathrm{C}} \mathrm{b}$ & $\delta_{\mathrm{H}}$, mult., $J(\mathrm{~Hz})$ & HMBC correlations & NOE correlations \\
\hline 2 & $106.0, \mathrm{C}$ & & \\
3 & $99.7, \mathrm{C}$ & & & \\
4 & $114.4, \mathrm{CH}$ & $6.86, \mathrm{~s}$ & 4,7 & \\
5 & $125.8, \mathrm{C}$ & & $8 \mathrm{~b}$ & $8 \mathrm{a}, 8 \mathrm{~b}$ \\
6 & $157.8, \mathrm{C}$ & & & 7,9 \\
7 & & $7.90, \mathrm{~d}(5.1)$ & 7,9 \\
$8 \mathrm{a}$ & $42.0, \mathrm{CH} 2$ & $3.79, \mathrm{dd}(13.7,4.0)$ & $8 \mathrm{a}, 8 \mathrm{~b}, 10 \mathrm{~b}, 11$ \\
$\mathrm{~b}$ & & $3.44, \mathrm{dd}(13.7,5.1)$ & & 9,11 \\
9 & $50.3, \mathrm{CH}$ & $4.70, \mathrm{ddd}(9.5,5.0,4.0)$ & $7,10 \mathrm{a}, 11$ & \\
$10 \mathrm{a}$ & $32.8, \mathrm{CH} 2$ & $2.25, \mathrm{ddd}(14.0,9.5,6.8)$ & 11 & $9,10 \mathrm{~b}, 12$ \\
$\mathrm{~b}$ & & $1.89, \mathrm{ddd}(14.0,7.6,5.0)$ & & $11,13-\mathrm{NH}$ \\
11 & $56.0, \mathrm{CH}$ & $4.39, \mathrm{dd}(7.6,6.8)$ & $10 \mathrm{a}, 12$ & 12 \\
12 & & $9.71, \mathrm{~s}$ & & \\
13 & $158.5, \mathrm{C}$ & & $9.12, \mathrm{brs}$ & \\
$13-\mathrm{NH}$ & & $9.12, \mathrm{brs}$ & 11,12 & \\
14 & & & $10 \mathrm{a}, 11,12$ & \\
15 & $174.9, \mathrm{C}$ & & & \\
\hline
\end{tabular}

${ }^{\mathrm{a}} 500.13 \mathrm{MHz}$ for ${ }^{1} \mathrm{H}$ and $125.76 \mathrm{MHz}$ for ${ }^{13} \mathrm{C}$. ${ }^{\mathrm{b}}$ Multiplicity and assignment from HSQC experiment. 
S104. HR ESI MS data of Agesamine B

Elemental Composition Report

Page 1

Single Mass Analysis
Tolerance $=5.0 \mathrm{mDa} / \mathrm{DBE}: \min =-1.5, \max =50.0$

m:

Number of isotope peaks used for $\mathrm{i}-\mathrm{FIT}=3$

Monoisotopic Mass, Odd and Even Electron Ions

778 formula(e) evaluated with 22 results within limits (up to 4 closest results for each mass)

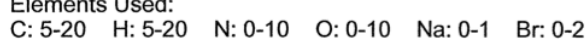

DK196.2

Dimitry Kovalerchik

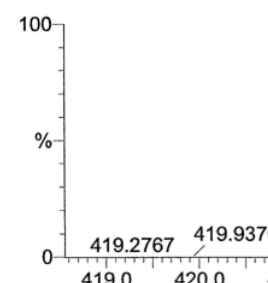

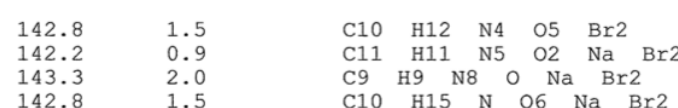

